\title{
The first synthesis of the ABC-ring system of 'upenamide
}

\author{
Jan Peter Schmidt, ${ }^{a}$ Sandra Beltran-Rodil, ${ }^{a}$ Rhona J. Cox, ${ }^{b}$ Graeme D. McAllister, ${ }^{a}$ Mark Reid, ${ }^{a}$ and Richard J. \\ K. Taylor. ${ }^{a *}$ \\ a) Department of Chemistry, University of York, York, YO10 5DD, UK. \\ b) Medicinal Chemistry, AstraZeneca R\&D Charnwood, Loughborough, LE11 5RH, UK.
}

\section{Representative Experimental Procedures and Supplementary Data}

Acetic acid (1R,6S)-6-hydroxymethyl-cyclohex-3-enylmethyl ester (-)-5.

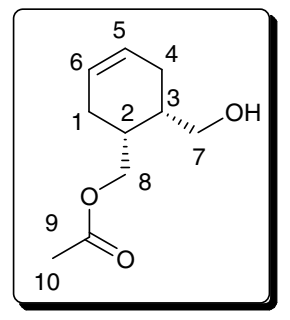

To a stirred emulsion of diacetate $7(21.0 \mathrm{~g}, 92.8 \mathrm{mmol})$ in phosphate buffer $(200 \mathrm{~mL}, \mathrm{pH} 7)$ at $\mathrm{rt}$ was added porcine pancreatic lipase (PPL, Sigma Type II, 100-400 units/mg, $2.00 \mathrm{~g}$ ). As the reaction proceeded, the $\mathrm{pH}$ was kept constant by the addition of $\mathrm{NaOH}(1 \mathrm{M})$ from an autoburette $(\mathrm{pH}$ set to 7.00). After $24 \mathrm{~h}$, approximately 80 $\mathrm{mL}$ of $\mathrm{NaOH}$ solution had been added (ca. $80 \%$ conversion) and the reaction had stopped. The reaction was then filtered through a pad of Celite ${ }^{\circledR}$ and the solids were washed with $\mathrm{Et}_{2} \mathrm{O}(200 \mathrm{~mL})$. The filtrate was extracted with $\mathrm{Et}_{2} \mathrm{O}(3 \times 100 \mathrm{~mL})$ and the combined organic layers were dried over $\mathrm{Na}_{2} \mathrm{SO}_{4}$. The solvent was removed in vacuo and the crude oil purified by column chromatography (eluting with $5-80 \% \mathrm{EtOAc/iso-hexane)} \mathrm{to} \mathrm{furnish} \mathrm{alcohol} \mathrm{(-)-}$ 5 as a colourless oil (13.5 g, 79\%); $R_{\mathrm{f}} 0.16$ (30\% EtOAc/PE); [a] $]_{D}-20.5$ (c 2.2, $\mathrm{CHCl}_{3}$ ) (Lit. ${ }^{4}$-19.0 (c 4.0, $\mathrm{CHCl}_{3}$ ); $\delta_{\mathrm{H}}\left(400 \mathrm{MHz}, \mathrm{CDCl}_{3}\right)$ 5.68-5.62 (2 H, m, H-5, H-6), $4.20\left(1 \mathrm{H}, \mathrm{dd}, J 11.0,6.0 \mathrm{~Hz}, \mathrm{H}-8_{\mathrm{a}}\right), 3.96(1 \mathrm{H}, \mathrm{dd}$, J, 11.0, 7.5 $\left.\mathrm{Hz}, \mathrm{H}-8_{b}\right), 3.72-3.57$ (2 H, m, H-7), 2.29-2.08 (4 H, m, H-1a, H-2, H-3, H-4a), 2.07 (3 H, s, H-9), 2.01-1.72 (3 H, m, $\left.\mathrm{H}-1_{b}, \mathrm{H}-4_{b}, \mathrm{OH}\right) .{ }^{1} \mathrm{H}-\mathrm{NMR}$ data were consistent with those published. ${ }^{1}$

((1R,6S)-6-Benzyloxymethyl-cyclohex-3-enyl)-methanol (+)-9.
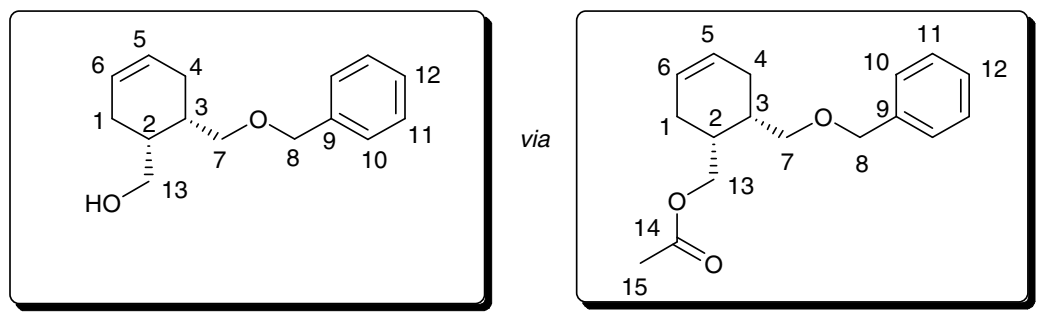

(a) To a stirred solution of alcohol (-)-5 (3.00 g, $16.3 \mathrm{mmol})$ in $\alpha, \alpha, \alpha$-trifluorotoluene $(40 \mathrm{~mL})$ was added magnesium oxide $(1.31 \mathrm{~g}, 32.6 \mathrm{mmol})$ and 2-benzyloxy- $N$-methylpyridinium triflate 8 (16.5 g, $48.9 \mathrm{mmol})$. The resulting slurry was stirred at $83^{\circ} \mathrm{C}$ for $21 \mathrm{~h}$. The reaction was then filtered through Celite ${ }^{\circledR}$ and the solids were washed with EtOAc $(50 \mathrm{~mL})$. The filtrate was concentrated under reduced pressure and the crude oil purified by column chromatography (eluting with $2-20 \% \mathrm{EtOAc/PE}$ ) to furnish the intermediate benzyl ether as a colourless 
oil (4.29 g, 96\%); $R_{\mathrm{f}} 0.52$ (30\% EtOAc/PE); [a $]_{D}+11.1$ (c 1.2, $\mathrm{CHCl}_{3}$ ); IR (neat) 3027, 2900, $2847\left(\mathrm{C}-\mathrm{H}_{\text {stretch }}\right), 1739$ $\left(\mathrm{C}=\mathrm{O}_{\text {stretch }}\right) ; \delta_{\mathrm{H}}\left(400 \mathrm{MHz}, \mathrm{CDCl}_{3}\right)$ 7.38-7.28 $(5 \mathrm{H}, \mathrm{m}, \mathrm{H}-10, \mathrm{H}-11, \mathrm{H}-12), 5.67-5.60(2 \mathrm{H}, \mathrm{m}, \mathrm{H}-5, \mathrm{H}-6), 4.51(2 \mathrm{H}, \mathrm{s}$, $\mathrm{H}-8), 4.11\left(1 \mathrm{H}, \mathrm{dd}, J 11.0,5.5 \mathrm{~Hz}, \mathrm{H}-7_{a}\right), 4.01\left(1 \mathrm{H}, \mathrm{dd}, J 11.0,8.0 \mathrm{~Hz}, \mathrm{H}-7_{b}\right), 3.50\left(1 \mathrm{H}, \mathrm{dd}, J 9.0,6.5 \mathrm{~Hz}, \mathrm{H}-13_{a}\right)$, $3.41\left(1 \mathrm{H}\right.$, dd, J 9.0, $\left.7.0 \mathrm{~Hz}, \mathrm{H}-13_{b}\right)$, 2.28-1.91 (9 H, m, H-1, H-2, H-3, H-4, H-15); $\delta_{\mathrm{C}}\left(100 \mathrm{MHz}, \mathrm{CDCl}_{3}\right) 171.9$ (C14), 138.9 (C-Ar quat), 128.8 (C-Ar), 127.9 (C-Ar), 126.1 (C-5/C-6), 125.4 (C-6/C-5), 73.3 (C-8), 71.3 (C-13), 65.3 (C-7), 34.7 (C-2/C-3), 33.6 (C-3/C-2), 26.63 (C-1/C-4), 26.61 (C-4/C-1), 20.9 (C-15); m/z (Cl) 275 (16), 215 (79) $[\mathrm{MH}]^{+}$; HRMS (Cl) calcd. For $\mathrm{C}_{17} \mathrm{H}_{23} \mathrm{O}_{3}, 275.1647$. Found: $[\mathrm{MH}]^{+}, 275.1648$ (-0.2 ppm error)].

(b) To a stirred solution of the intermediate benzyl ether $(3.97 \mathrm{~g}, 14.5 \mathrm{mmol})$ in $\mathrm{MeOH}(60 \mathrm{~mL})$ and water $(30 \mathrm{~mL})$ at $0{ }^{\circ} \mathrm{C}$ was added lithium hydroxide monohydrate $(670 \mathrm{mg}, 15.9 \mathrm{mmol})$. The ice-bath was removed and the reaction mixture was stirred for $45 \mathrm{~min}$. The $\mathrm{MeOH}$ was then removed in vacuo and the crude aqueous layer partitioned between EtOAc $(100 \mathrm{~mL})$ and brine $(100 \mathrm{~mL})$. The aqueous phase was extracted using EtOAc $(2 \mathrm{x}$ $100 \mathrm{~mL}$ ). The combined organic layers were dried over $\mathrm{Na}_{2} \mathrm{SO}_{4}$ and concentrated in vacuo, to give the alcohol (+)-9 (3.37 g, 100\%) without any further purification; $R_{\mathrm{f}} 0.24\left(50 \% \mathrm{Et}_{2} \mathrm{O} / \mathrm{PE}\right) ;[\alpha]_{D}+3.3\left(\mathrm{c} 1.8, \mathrm{CHCl}_{3}\right) ; \delta_{\mathrm{H}}(400$ $\left.\mathrm{MHz}, \mathrm{CDCl}_{3}\right)$ 7.38-7.27 (5 H, m, H-10, H-11, H-12), 5.64-5.56 (2 H, m, H-5, H-6), $4.55\left(1 \mathrm{H}, \mathrm{d}, \mathrm{J} 12.0 \mathrm{~Hz}, \mathrm{H}-8_{a}\right)$, $4.52\left(1 \mathrm{H}, \mathrm{d}, J 12.0 \mathrm{~Hz}, \mathrm{H}-8_{b}\right), 3.67-3.53\left(3 \mathrm{H}, \mathrm{m}, \mathrm{H}-7_{a}, \mathrm{H}-13\right), 3.41\left(1 \mathrm{H}, \mathrm{dd}, J 9.5,4.5 \mathrm{~Hz}, \mathrm{H}-7_{b}\right), 3.55(1 \mathrm{H}, \mathrm{br} \mathrm{s}$, $\mathrm{OH})$, 2.27-1.93 (6 H, m, H-1, H-2, H-3, H-4). ${ }^{1} \mathrm{H}-\mathrm{NMR}$ data were consistent with those reported in the literature. ${ }^{5}$

Methyl-(1R,6S)-6-(benzyloxymethyl)cyclohex-3-enecarboxylate (-)-10.

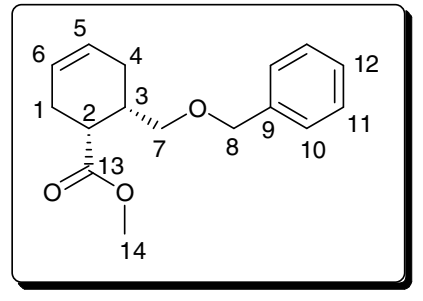

The crude alcohol (+)-9 (14.0 g, $60.3 \mathrm{mmol})$ was dissolved in acetone $(200 \mathrm{~mL})$ and stirred at $5{ }^{\circ} \mathrm{C}$. To this stirred solution was then added freshly prepared Jones' reagent until a dark red colour persisted for at least 10 min. Stirring was continued for another 30 min before propan-2-ol was added slowly, so as to maintain the internal temperature at or below $10^{\circ} \mathrm{C}$. The reaction was left to warm to rt before the green precipitate was filtered off. The filtrate was concentrated in vacuo and the green residue re-dissolved in $\mathrm{MeOH}(100 \mathrm{~mL})$. To this was added at $0{ }^{\circ} \mathrm{C}$ acetyl chloride $(2.6 \mathrm{~mL}, 36.2 \mathrm{mmol})$. The final solution was subsequently stirred at $\mathrm{rt}$ for $14 \mathrm{~h}$. After this time, the $\mathrm{MeOH}$ was removed in vacuo and the yellow residue dissolved in $\mathrm{CH}_{2} \mathrm{Cl}_{2}(100 \mathrm{~mL})$. The organic solution was washed twice with aqueous $\mathrm{NaHCO}_{3}$ (sat., $50 \mathrm{~mL}$ ). The organic layer was then dried over $\mathrm{Na}_{2} \mathrm{SO}_{4}$ and concentrated under reduced pressure. The crude oil was purified by column chromatography (eluting with $30 \%$ $\left.\mathrm{Et}_{2} \mathrm{O} / \mathrm{PE}\right)$ to give the methyl ester (-)-10 as a colourless oil $(11.3 \mathrm{~g}, 72 \%) ; R_{\mathrm{f}} 0.56\left(50 \% \mathrm{Et}_{2} \mathrm{O} / \mathrm{PE}\right) ;[\alpha]_{D}-15.5$ (c 1.1 , $\left.\mathrm{CHCl}_{3}\right)$; IR (neat) 3027, 2903, $2851\left(\mathrm{C}-\mathrm{H}_{\text {stretch }}\right), 1734\left(\mathrm{C}=\mathrm{O}_{\text {stretch }}\right) ; \delta_{\mathrm{H}}\left(400 \mathrm{MHz}, \mathrm{CDCl}_{3}\right)$ 7.36-7.27 $(5 \mathrm{H}, \mathrm{m}, \mathrm{H}-10, \mathrm{H}-$ 11, H-12), 5.67-5.60 (2 H, m, H-5, H-6), 4.46 (2 H, s, H-8), 3.59 (3 H, s, H-14), 3.49 (1 H, dd, J 9.0, 5.0 Hz, H-7a), $3.42\left(1 \mathrm{H}, \mathrm{dd}, \mathrm{J} 9.0,7.0 \mathrm{~Hz}, \mathrm{H}-7_{b}\right), 2.82-2.77(1 \mathrm{H}, \mathrm{m}, \mathrm{H}-2)$, 2.61-2.53 (1 H, m, H-3), 2.36-2.19 (3 H, m, H-1, H-4a), 2.11-2.04 (1 H, m, H-4 $)_{b}$; $\delta_{\mathrm{C}}\left(100 \mathrm{MHz}, \mathrm{CDCl}_{3}\right) 175.5$ (C-13), $138.9\left(\mathrm{C}-\mathrm{Ar}_{\text {quat }}\right), 128.7$ (C-Ar), 128.1 (C-Ar), 127.9 (C-Ar), 125.7 (C-5/C-6), 125.3 (C-6/C-5), 73.3 (C-8), 70.7 (C-7), 51.4 (C-14), 39.8 (C-2), 34.5 (C-3), 27.0 (C-4), $24.4(\mathrm{C}-1) ; \mathrm{m} / \mathrm{z}(\mathrm{Cl}) 261(100)[\mathrm{MH}]^{+}$; [HRMS (Cl) calcd. For $\mathrm{C}_{16} \mathrm{H}_{21} \mathrm{O}_{3}, 261.1491$. Found: $[\mathrm{MH}]^{+}, 261.1492(-0.6$ ppm error)]. 
Methyl-(1R,6S)-6-(benzyloxymethyl)-1-(3-(4-methoxybenzyloxy)propyl)cyclohex-3-enecarboxylate (+)12.

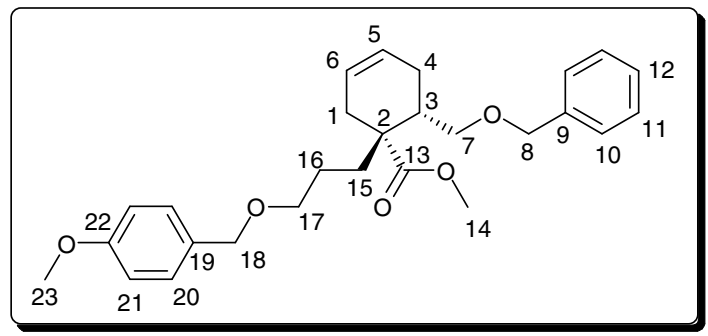

To a stirred solution of ester $(+)-10(700 \mathrm{mg}, 2.70 \mathrm{mmol})$ in THF $(7 \mathrm{~mL})$ at $-78{ }^{\circ} \mathrm{C}$ was added LiHMDS $(1 \mathrm{M}$ in THF, $2.83 \mathrm{~mL}, 2.83 \mathrm{mmol}$ ) dropwise. The resulting solution was stirred at that temperature for $2 \mathrm{~h}$ before a solution of iodide $11(1.06 \mathrm{~g}, 2.96 \mathrm{mmol})$ in THF $(3 \mathrm{~mL})$ was introduced. The final solution was allowed to stir at $78{ }^{\circ} \mathrm{C}$ for $15 \mathrm{~min}$ after which the cooling-bath was removed and the reaction allowed to warm to rt at which it was stirred for $18 \mathrm{~h}$. The reaction was quenched by the careful addition of water and then partitioned between aqueous $\mathrm{NH}_{4} \mathrm{Cl}$ (sat., $25 \mathrm{~mL}$ ) and EtOAc $(25 \mathrm{~mL})$. The aqueous layer was extracted using EtOAc $(3 \times 25 \mathrm{~mL})$ and the combined organic phases were dried over $\mathrm{Na}_{2} \mathrm{SO}_{4}$ and concentrated in vacuo. The crude oil was purified by column chromatography (eluting with $20 \% \mathrm{Et}_{2} \mathrm{O} / \mathrm{PE}$ ) to give the title compound (+)-12 as a colourless oil (1.04 $\mathrm{g}$, 88\%); $R_{\mathrm{f}} 0.24\left(30 \% \mathrm{Et}_{2} \mathrm{O} / \mathrm{PE}\right) ;[\alpha]_{D}+31.5$ (c 1.3, $\left.\mathrm{CHCl}_{3}\right)$; IR (neat) 3026, 2929, $2854\left(\mathrm{C}-\mathrm{H}_{\text {stretch }}\right), 1728\left(\mathrm{C}=\mathrm{O}_{\text {stretch }}\right)$; $\delta_{\mathrm{H}}\left(400 \mathrm{MHz}, \mathrm{CDCl}_{3}\right)$ 7.30-7.17 $(7 \mathrm{H}, \mathrm{m}, \mathrm{H}-10, \mathrm{H}-11, \mathrm{H}-12, \mathrm{H}-20), 6.81(2 \mathrm{H}, \mathrm{d}, J 9.0 \mathrm{~Hz}, \mathrm{H}-21), 5.54-5.48(2 \mathrm{H}, \mathrm{m}$, $\mathrm{H}-5, \mathrm{H}-6), 4.32(2 \mathrm{H}, \mathrm{s}, \mathrm{H}-8), 4.34(2 \mathrm{H}, \mathrm{s}, \mathrm{H}-18)$, 3.74 (3 H, s, H-23), 3.50 (3 H, s, H-14), 3.35-3.26 (4 H, m, H-7, $\mathrm{H}-17$ ), 2.33-2.17 (3 H, m, H-1 $\left., \mathrm{H}-3, \mathrm{H}-4_{a}\right), 2.04-1.97\left(2 \mathrm{H}, \mathrm{m}, \mathrm{H}-1_{b}, \mathrm{H}-4_{b}\right), 1.61-1.47\left(3 \mathrm{H}, \mathrm{m}, \mathrm{H}-15, \mathrm{H}-16_{a}\right), 1.37-$ $1.26\left(1 \mathrm{H}, \mathrm{m}, \mathrm{H}-16_{b}\right) ; \delta_{\mathrm{C}}\left(100 \mathrm{MHz}, \mathrm{CDCl}_{3}\right) 177.3$ (C-13), 159.6 (C-22), 138.8 (C-9), 130.9 (C-19), 129.6 (C-Ar), 128.6 (C-Ar), 128.0 (C-Ar), 127.8(C-Ar), 124.8, C-5/C-6), 124.5 (C-6/C-5), 114.0 (C-21), 73.2 (C-8), 72.4 (C-18), 71.3 (C-17), 70.2 (C-7), 55.2 (C-23), 51.4 (C-14), 45.6 (C-2), 39.0 (C-3), 32.0 (C-15), 27.2 (C-4), 24.8 (C-1), 24.2 (C-16); m/z (Cl) 439 (6) [MH] $]^{+}$; [HRMS (Cl) calcd. For $\mathrm{C}_{27} \mathrm{H}_{35} \mathrm{O}_{5}, 439.2495$. Found: $[\mathrm{MH}]^{+}, 439.2488$ (-0.7 ppm error)].

$\{(1 \mathrm{R}, 6 \mathrm{~S})-6-$ Benzyloxymethyl-1-[3-(4-methoxy-benzyloxy)-propyl]-cyclohex-3-enyl\}-methanol (+)-13.

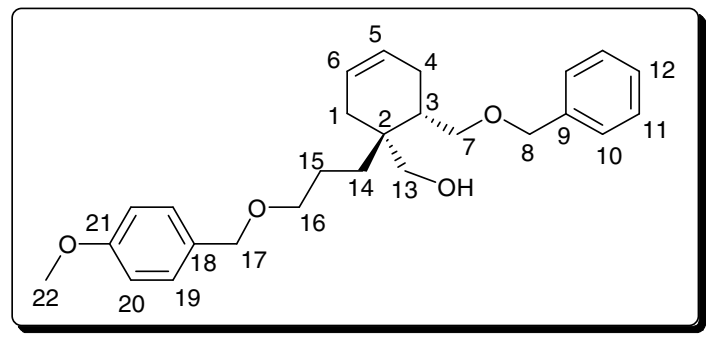

To a stirred solution of methyl ester (+)-12 (10.6 g, $24.2 \mathrm{mmol})$ in $\mathrm{CH}_{2} \mathrm{Cl}_{2}(100 \mathrm{~mL})$ at $-20{ }^{\circ} \mathrm{C}$ was added DIBAL-H (1 $\mathrm{M}$ in $\mathrm{CH}_{2} \mathrm{Cl}_{2}, 50.0 \mathrm{~mL}, 48.3 \mathrm{mmol}$ ). The resulting solution was stirred at $-78{ }^{\circ} \mathrm{C}$ for $30 \mathrm{~min}$. The reaction was then quenched by the careful addition of EtOAc $(10 \mathrm{~mL})$ at $-15^{\circ} \mathrm{C}$ and the reaction mixture was transferred into a round bottom flask containing Rochelle's salt solution $(20 \% \mathrm{w} / \mathrm{w}, 500 \mathrm{~mL})$. After stirring vigorously for $3 \mathrm{~h}$, the reaction mixture was partitioned and the aqueous phase extracted with $\mathrm{CH}_{2} \mathrm{Cl}_{2}(3 \times 100 \mathrm{~mL})$. The combined organic phases were concentrated in vacuo to furnish the title compound (+)-13 as a colourless oil (9.92 $\mathrm{g}$, $100 \%$ ); $R_{\mathrm{f}} 0.36$ (80\% Et $\left.\mathrm{E}_{2} \mathrm{O} / \mathrm{PE}\right) ;[\alpha]_{D}+18.8$ (c 1.7, $\mathrm{CHCl}_{3}$ ); IR (neat) $3431\left(\mathrm{O}-\mathrm{H}_{\text {stretch }}\right), 3023,2922,2855\left(\mathrm{C}-\mathrm{H}_{\text {stretch }}\right)$; $\delta_{\mathrm{H}}\left(400 \mathrm{MHz}, \mathrm{CDCl}_{3}\right)$ 7.29-7.18 $(7 \mathrm{H}, \mathrm{m}, \mathrm{H}-10, \mathrm{H}-11, \mathrm{H}-12, \mathrm{H}-19), 6.80(2 \mathrm{H}, \mathrm{d}, J 9.0 \mathrm{~Hz}, \mathrm{H}-20), 5.52(1 \mathrm{H}, \mathrm{br}$ d, J $10.5 \mathrm{~Hz}, \mathrm{H}-5), 5.45(1 \mathrm{H}, \mathrm{br} d, J 10.5 \mathrm{~Hz}, \mathrm{H}-6), 4.45\left(1 \mathrm{H}, \mathrm{d}, J 12.0 \mathrm{~Hz}, \mathrm{H}-8_{a}\right), 4.41\left(1 \mathrm{H}, \mathrm{d}, J 12.0 \mathrm{~Hz}, \mathrm{H}-8_{b}\right), 4.35$ 
(2 H, s, H-17), 3.71 (3 H, s, H-22), 3.57 (1 H, d, J $\left.12.0 \mathrm{~Hz}, \mathrm{H}-13_{a}\right), 3.52$ (1 H, dd, J 9.5, $3.5 \mathrm{~Hz}, \mathrm{H}-7_{\mathrm{a}}$ ), $3.41-3.31$ (3 $\left.\mathrm{H}, \mathrm{m}, \mathrm{H}-7_{b}, \mathrm{H}-16\right)$, $3.14\left(1 \mathrm{H}, \mathrm{d}, J 12.0 \mathrm{~Hz}, \mathrm{H}-13_{b}\right), 2.20-2.12\left(1 \mathrm{H}, \mathrm{m}, \mathrm{H}-4_{a}\right), 2.03-1.99\left(1 \mathrm{H}, \mathrm{m}, \mathrm{H}-4_{b}\right), 1.85-1.77(2$ $\left.\mathrm{H}, \mathrm{m}, \mathrm{H}-3, \mathrm{H}-15_{a}\right), 1.69-1.43\left(4 \mathrm{H}, \mathrm{m}, \mathrm{H}-1, \mathrm{H}-14_{a}, \mathrm{H}-15_{b}\right), 1.30-1.27\left(1 \mathrm{H}, \mathrm{m}, \mathrm{H}-1_{b}\right) ; \delta_{\mathrm{C}}\left(100 \mathrm{MHz}, \mathrm{CDCl}_{3}\right) 159.6(\mathrm{C}-$ 21), 137.8 (C-Ar quat), 131.0 (C-18), 129.6 (C-Ar), 128.8 (C-Ar), 128.2 (C-Ar), 128.1 (C-Ar), 125.8 (C-5), 125.1 (C6), 114.0 (C-20), 73.5 (C-8), 72.5 (C-17), 70.92 (C-16), 70.87 (C-7), 64.8 (C-13), 55.2 (C-22), 39.4 (C-3), 38.5 (C2), 33.0 (C-15), 31.0 (C-1), 26.4 (C-4), 23.0 (C-14); m/z (Cl) 411 (5) [MH] ; [HRMS (Cl) calcd. For $\mathrm{C}_{26} \mathrm{H}_{35} \mathrm{O}_{4}$, 411.2535. Found: $[\mathrm{MH}]^{+}, 411.2532$ (0.8 ppm error)].

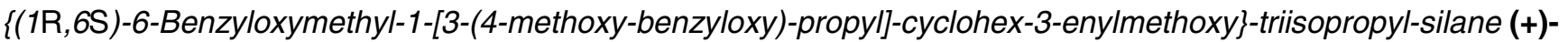
14.

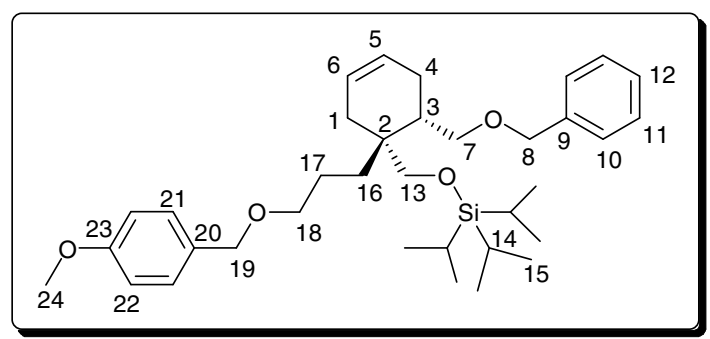

To a stirred solution of alcohol (+)-13 $(9.92 \mathrm{~g}, 24.2 \mathrm{mmol})$ in $\mathrm{CH}_{2} \mathrm{Cl}_{2}(200 \mathrm{~mL})$ at $0{ }^{\circ} \mathrm{C}$ was added 2,6-lutidine (7.0 $\mathrm{mL}, 60.5 \mathrm{mmol}$ ) followed by triisopropylsilyl trifluoromethane-sulfonate $(9.8 \mathrm{~mL}, 36.3 \mathrm{mmol})$. The resulting solution was stirred at rt for $5 \mathrm{~h}$. The reaction mixture was then diluted with further $\mathrm{CH}_{2} \mathrm{Cl}_{2}(100 \mathrm{~mL})$ and water $(300 \mathrm{~mL})$. The aqueous phase was extracted using $\mathrm{CH}_{2} \mathrm{Cl}_{2}(2 \times 100 \mathrm{~mL})$ and the combined organic layers dried over $\mathrm{Na}_{2} \mathrm{SO}_{4}$. The solvent was removed in vacuo and the crude product purified by column chromatography (eluting with $\left.10-50 \% \mathrm{Et}_{2} \mathrm{O} / \mathrm{PE}\right)$ to furnish the title compound $(+)-14$ as a colourless oil $(13.3 \mathrm{~g}, 97 \%) ; R_{\mathrm{f}} 0.51(30 \%$ $\mathrm{Et}_{2} \mathrm{O} / \mathrm{PE}$ ); $[\alpha]_{D}+19.2$ (c 1.2, $\left.\mathrm{CHCl}_{3}\right)$; IR (neat) 2941, $2864\left(\mathrm{C}-\mathrm{H}_{\text {stretch }}\right) ; \delta_{\mathrm{H}}\left(400 \mathrm{MHz}, \mathrm{CDCl}_{3}\right) 7.32(4 \mathrm{H}, \mathrm{d}, J 4.5 \mathrm{~Hz}$, $\mathrm{H}-10, \mathrm{H}-11), 7.29-7.24(3 \mathrm{H}, \mathrm{m}, \mathrm{H}-12, \mathrm{H}-21), 6.86(2 \mathrm{H}, \mathrm{d}, \mathrm{J} 8.5 \mathrm{~Hz}, \mathrm{H}-22), 5.63(1 \mathrm{H}, \mathrm{br} \mathrm{d}, J 10.0 \mathrm{~Hz}, \mathrm{H}-5), 5.56$ $(1 \mathrm{H}$, br d, J $10.0 \mathrm{~Hz}, \mathrm{H}-6), 4.51\left(1 \mathrm{H}, \mathrm{d}, J 12.0 \mathrm{~Hz}, \mathrm{H}-8_{a}\right), 4.43\left(1 \mathrm{H}, \mathrm{d}, J 12.0 \mathrm{~Hz}, \mathrm{H}-8_{b}\right), 4.42(2 \mathrm{H}, \mathrm{s}, \mathrm{H}-19), 3.80$ (3 H, s, H-24), $3.68\left(1 \mathrm{H}, \mathrm{d}, \mathrm{J} 10.0 \mathrm{~Hz}, \mathrm{H}-13_{a}\right), 3.67\left(1 \mathrm{H}\right.$, dd, J 9.0, 3.5 Hz, H-7a), 3.47-3.39 (4 H, m, H-7, $\mathrm{H}-13_{b}$, $\mathrm{H}-18), 2.33\left(1 \mathrm{H}, \mathrm{br} d, J 16.5 \mathrm{~Hz}, \mathrm{H}-4_{a}\right), 2.07-1.96\left(2 \mathrm{H}, \mathrm{m}, \mathrm{H}-3, \mathrm{H}-4_{b}\right), 1.90\left(1 \mathrm{H}, \mathrm{br} \mathrm{d}, J 18.0 \mathrm{~Hz}, \mathrm{H}-1_{a}\right), 1.76(1 \mathrm{H}$, br d, J $\left.18.0 \mathrm{~Hz}, \mathrm{H}-1_{b}\right), 1.63-1.47(4 \mathrm{H}, \mathrm{m}, \mathrm{H}-16, \mathrm{H}-17), 1.02-1.01(21 \mathrm{H}, \mathrm{m}, \mathrm{H}-14, \mathrm{H}-15) ; \delta_{\mathrm{C}}\left(100 \mathrm{MHz}, \mathrm{CDCl}_{3}\right)$ 159.6 (C-23), 139.3 (C-Ar $\left.{ }_{\text {quat }}\right), 131.3$ (C-20), 129.5 (C-Ar), 128.7 (C-Ar), 127.9 (C-Ar), 127.8 (C-Ar), 125.9 (C-5), 125.5 (C-6), 114.0 (C-22), 73.1 (C-8), 72.5 (C-19), 71.4 (C-7), 71.1 (C-16), 66.5 (C-13), 55.3 (C-24), 38.5 (C-3), 31.9 (C-1), 30.8 (C-16), 26.5 (C-4), 23.2 (C-17), 17.9 (C-15), 11.8 (C-14); m/z (FAB) 589 (21) [MNa] ; [HRMS (FAB) calcd. For $\mathrm{C}_{35} \mathrm{H}_{54} \mathrm{O}_{4} \mathrm{Si}, 589.3684$. Found: [MNa] $]^{+}, 589.3686$ (0.4 ppm error)].

3-((1R,6S)-6-Benzyloxymethyl-1-(triisopropylsilanyloxy)methyl-cyclohex-3-enyl)-propan-1-ol (+)-15.

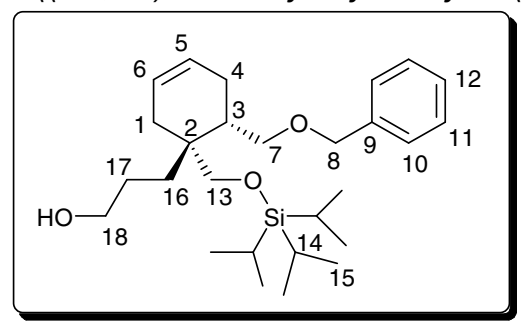

To a stirred solution of p-methoxybenzyl ether $(+)-14(13.3 \mathrm{~g}, 23.5 \mathrm{mmol})$ in $\mathrm{CH}_{2} \mathrm{Cl}_{2}(160 \mathrm{~mL})$ and water $(8 \mathrm{~mL})$ at rt was added 2,3-dichloro-5,6-dicyanobenzoquinone (5.86 g, $25.8 \mathrm{mmol})$. The final dark green solution was stirred 
at $\mathrm{rt}$ for $15 \mathrm{~min}$ (until yellow). The reaction mixture was then diluted with further $\mathrm{CH}_{2} \mathrm{Cl}_{2}(40 \mathrm{~mL}$ ) and aqueous $\mathrm{NaHCO}_{3}(10 \%, 150 \mathrm{~mL})$. The aqueous phase was extracted using $\mathrm{CH}_{2} \mathrm{Cl}_{2}(2 \times 50 \mathrm{~mL})$ and the combined organic layers were dried over $\mathrm{Na}_{2} \mathrm{SO}_{4}$. The solvent was removed in vacuo and the crude product purified by column chromatography (eluting with $\left.10-100 \% \mathrm{Et}_{2} \mathrm{O} / \mathrm{PE}\right)$ to furnish the title compound (+)-15 as a colourless oil $(9.85 \mathrm{~g}$, 94\%); Found C, 72.64\%; $\mathrm{H}, 10.63 \% . \mathrm{C}_{27} \mathrm{H}_{46} \mathrm{O}_{3} \mathrm{Si}$ requires $\mathrm{C}, 72.59 \% ; \mathrm{H}, 10.38 \% ; R_{\mathrm{f}} 0.40\left(60 \% \mathrm{Et}_{2} \mathrm{O} / \mathrm{PE}\right) ;[\alpha]_{D}$ +24.0 (c 1.0, $\left.\mathrm{CHCl}_{3}\right)$; IR (neat) $3352\left(\mathrm{O}-\mathrm{H}_{\text {stretch }}\right), 3025,2941,2866\left(\mathrm{C}-\mathrm{H}_{\text {stretch }}\right) ; \delta_{\mathrm{H}}\left(400 \mathrm{MHz}, \mathrm{CDCl}_{3}\right)$ 7.44-7.17 (5 $\mathrm{H}, \mathrm{m}, \mathrm{H}-10, \mathrm{H}-11, \mathrm{H}-12), 5.56(1 \mathrm{H}, \mathrm{br} \mathrm{d}, J 10.0 \mathrm{~Hz}, \mathrm{H}-5), 5.49(1 \mathrm{H}, \mathrm{br} \mathrm{d}, J 10.0 \mathrm{~Hz}, \mathrm{H}-6), 4.41(1 \mathrm{H}, \mathrm{d}, J 12.0 \mathrm{~Hz}$, H-8 $)_{a}$ ), $4.39\left(1 \mathrm{H}, \mathrm{d}, J 12.0 \mathrm{~Hz}, \mathrm{H}-8_{b}\right), 3.61\left(1 \mathrm{H}, \mathrm{d}, J 10.0 \mathrm{~Hz}, \mathrm{H}-13_{a}\right), 3.60\left(1 \mathrm{H}, \mathrm{dd}, J\right.$ 9.0, $\left.3.5 \mathrm{~Hz}, \mathrm{H}-7_{a}\right), 3.54-3.51$ $(2 \mathrm{H}, \mathrm{m}, \mathrm{H}-18), 3.42\left(1 \mathrm{H}, \mathrm{d}, J 10.0 \mathrm{~Hz}, \mathrm{H}-13_{b}\right), 3.36\left(1 \mathrm{H}, \mathrm{dd}, J\right.$ 9.0, $\left.9.0 \mathrm{~Hz}, \mathrm{H}-7_{b}\right), 2.25(1 \mathrm{H}, \mathrm{br} d, J, 18.0 \mathrm{~Hz}, \mathrm{H}-$ 4a), 2.02-1.89 (2 H, m, H-3, H-4 $)$ ), $1.83\left(1 \mathrm{H}, \mathrm{br} \mathrm{d}, \mathrm{J} 18.0 \mathrm{~Hz}, \mathrm{H}-1_{a}\right), 1.70\left(1 \mathrm{H}, \mathrm{br} \mathrm{d}, J 18.0 \mathrm{~Hz}, \mathrm{H}-1_{b}\right), 1.50-1.42(4$ $\mathrm{H}, \mathrm{m}, \mathrm{H}-16, \mathrm{H}-17), 1.32(1 \mathrm{H}, \mathrm{br} \mathrm{s}, \mathrm{OH}), 1.06-0.99(21 \mathrm{H}, \mathrm{m}, \mathrm{H}-14, \mathrm{H}-15)$; $\delta_{\mathrm{C}}\left(100 \mathrm{MHz}, \mathrm{CDCl}_{3}\right) 139.3\left(\mathrm{C}-\mathrm{Ar}_{\text {quat }}\right.$ ), 128.7 (C-Ar), 128.0 (C-Ar), 127.8 (C-Ar), 125.9 (C-5), 125.5 (C-6), 73.1 (C-8), 71.3 (C-7), 66.5 (C-13), 64.0 (C18), 38.42 (C-2), 38.35 (C-3), 31.9 (C-1), 30.4 (C-17), 26.5 (C-4), 26.3 (C-16), 17.9 (C-15), 11.8 (C-14); m/z (Cl) 447 (73) $[\mathrm{MH}]^{+}$; [HRMS (Cl) calcd. For $\mathrm{C}_{27} \mathrm{H}_{47} \mathrm{O}_{3} \mathrm{Si}$, 447.3294. Found: [MH] ${ }^{+}, 447.3297$ (-0.5 ppm error)].

[(1R,6S)-1-(3-Azido-propyl)-6-benzyloxymethyl-cyclohex-3-enylmethoxy]-triisopropyl-silane (+)-16.

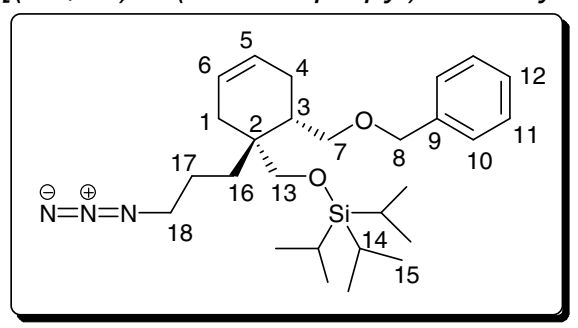

To a stirred solution of triphenylphosphine $(6.35 \mathrm{~g}, 24.2 \mathrm{mmol})$ in THF $(90 \mathrm{~mL})$ at $0{ }^{\circ} \mathrm{C}$ was added diisopropyl azodicarboxylate $(4.76 \mathrm{~mL}, 24.2 \mathrm{mmol})$. The resulting solution was stirred for $15 \mathrm{~min}$ until a white precipitate formed. To this was then added a solution of alcohol (+)-15 $(9.00 \mathrm{~g}, 20.2 \mathrm{mmol})$ in THF (40 mL) followed by diphenylphosphoryl azide $(5.22 \mathrm{~mL}, 24.2 \mathrm{mmol})$. The final solution was stirred at $\mathrm{rt}$ for $5 \mathrm{~h}$. The solvent was then removed in vacuo and the yellow crude oil purified by column chromatography (eluting with 1-3\% $\mathrm{Et}_{2} \mathrm{O} /$ iso $^{-}$ hexane) to furnish azide (+)-16 as a colourless oil (7.98 g, 83\%); $R_{\mathrm{f}} 0.32\left(5 \% \mathrm{Et}_{2} \mathrm{O} / \mathrm{PE}\right) ;[\alpha]_{D}+26.2\left(\mathrm{c} 1.3, \mathrm{CHCl}_{3}\right)$;

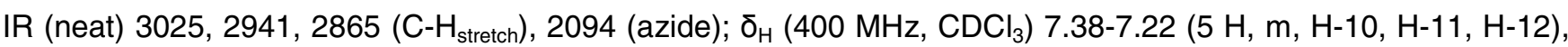
$5.55(1 \mathrm{H}, \mathrm{br} d, J 10.0 \mathrm{~Hz}, \mathrm{H}-5), 5.48(1 \mathrm{H}$, br d, J $10.0 \mathrm{~Hz}, \mathrm{H}-6), 4.43\left(1 \mathrm{H}, \mathrm{d}, J 12.0 \mathrm{~Hz}, \mathrm{H}-8_{a}\right), 4.37(1 \mathrm{H}, \mathrm{d}, J$ $\left.12.0 \mathrm{~Hz}, \mathrm{H}-8_{b}\right), 3.60\left(1 \mathrm{H}, \mathrm{d}, J 10.0 \mathrm{~Hz}, \mathrm{H}-13_{a}\right), 3.56\left(1 \mathrm{H}, \mathrm{dd}, J 9.0,4.0 \mathrm{~Hz}, \mathrm{H}-7_{a}\right), 3.48\left(1 \mathrm{H}, \mathrm{d}, J 10.0 \mathrm{~Hz}, \mathrm{H}-13_{b}\right)$, $3.40\left(1 \mathrm{H}, \mathrm{dd}, J\right.$ 9.0, $\left.9.0 \mathrm{~Hz}, \mathrm{H}-7_{b}\right), 3.21(2 \mathrm{H}, \mathrm{t}, J 7.0 \mathrm{~Hz}, \mathrm{H}-18), 2.22\left(1 \mathrm{H}, \mathrm{br}\right.$ d, J $\left.18.0 \mathrm{~Hz}, \mathrm{H}-4_{a}\right), 2.12-1.86(2 \mathrm{H}$, m, H-3, H-4 $)$, $1.81\left(1 \mathrm{H}, \mathrm{br} d, J 18.0 \mathrm{~Hz}, \mathrm{H}-1_{a}\right), 1.71\left(1 \mathrm{H}, \mathrm{br} \mathrm{d}, J 18.0 \mathrm{~Hz}, \mathrm{H}-1_{b}\right), 1.67-1.47(4 \mathrm{H}, \mathrm{m}, \mathrm{H}-16, \mathrm{H}-17)$, 1.08-0.99 (21 H, m, H-14, H-15); $\delta_{\mathrm{C}}\left(100 \mathrm{MHz}, \mathrm{CDCl}_{3}\right) 139.2$ (C-Ar quat), 128.7 (C-Ar), 128.0 (C-Ar), 127.8 (C-Ar), 125.9 (C-5), 125.3 (C-6), 73.2 (C-8), 71.2 (C-7), 66.5 (C-13), 52.4 (C-18), 38.5 (C-2), 38.2 (C-3), 31.9 (C-1), 27.1 (C-17), 26.4 (C-4), 22.7 (C-16), 17.9 (C-15), 11.7 (C-14); m/z (Cl) 472 (55) [MH] ${ }^{+}, 444$ (100) [MH(-N $]_{2}^{+}$; [HRMS (Cl) calcd. For $\mathrm{C}_{27} \mathrm{H}_{46} \mathrm{~N}_{3} \mathrm{O}_{2} \mathrm{Si}, 472.3359$. Found: [MH] $]^{+}, 472.3372$ (-2.6 ppm error)].

$\mathrm{N}-[3-((1 \mathrm{R}, 6 \mathrm{~S})-6-B e n z y l o x y m e t h y l-1-t r i i s o p r o p y l s i l a n y l o x y m e t h y l-c y c l o h e x-3-e n y l)-p r o p y l]-2-((\mathrm{S})-2,2-d i m e t h y l-1,3-$ dioxolan-4-yl)-acetamide (+)-18. 


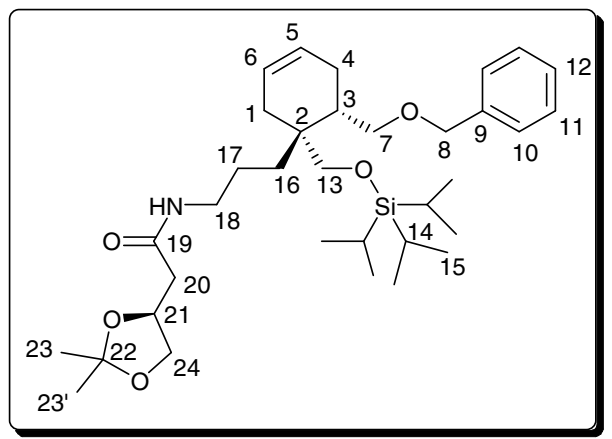

To a stirred solution of azide (+)-16 $(6.84 \mathrm{~g}, 14.5 \mathrm{mmol})$ in THF $(50 \mathrm{~mL})$ and water $(3.3 \mathrm{~mL})$ was added triphenylphosphine $(4.18 \mathrm{~g}, 15.6 \mathrm{mmol})$. The resulting solution was stirred at reflux for $2 \mathrm{~h}$. TLC analysis showed complete reduction of the azide and formation of a very polar species. The solvent was removed in vacuo. The crude solid was then dissolved in $\mathrm{CH}_{2} \mathrm{Cl}_{2}(20 \mathrm{~mL})$ and stirred over $\mathrm{Na}_{2} \mathrm{SO}_{4}(2 \mathrm{~g})$ for $15 \mathrm{~min}$. The solids were then filtered off and washed with further $\mathrm{CH}_{2} \mathrm{Cl}_{2}$. The filtrate was concentrated to give a white solid which was dissolved in THF $(80 \mathrm{~mL})$ and cooled to $0^{\circ} \mathrm{C}$. To this was added acid $(\mathrm{S})-17(2.79 \mathrm{~g}, 17.42 \mathrm{mmol})$ and $\mathrm{N}, \mathrm{N}$ diisopropylethylamine $(5.10 \mathrm{~mL}, 29.0 \mathrm{mmol})$ and $\mathrm{T}^{\circledR} \mathrm{P}^{\circledR}(50 \% \mathrm{w} / \mathrm{w}$ in THF, $13.9 \mathrm{~g}, 21.8 \mathrm{mmol})$. The final solution was allowed to stir at rt for $16 \mathrm{~h}$. The solvent was removed in vacuo and the crude material partitioned between EtOAc $(50 \mathrm{~mL})$ and water $(50 \mathrm{~mL})$. The organic layer was washed sequentially with aqueous $\mathrm{HCl}(10 \%, 50 \mathrm{~mL})$, aqueous $\mathrm{NaHCO}_{3}$ (sat., $50 \mathrm{~mL}$ ) and brine $\left(50 \mathrm{~mL}\right.$ ), then dried over $\mathrm{Na}_{2} \mathrm{SO}_{4}$ and concentrated in vacuo. The yellow crude oil was purified by column chromatography (eluting with $30 \%$ EtOAc/PE) to furnish the amide (+)-18 as a colourless oil (7.36 g, 86\%); $R_{\mathrm{f}} 0.30$ (50\% EtOAc/iso-hexane); $[\alpha]_{D}+12.7$ (c 1.1, $\left.\mathrm{CHCl}_{3}\right)$; IR (neat) 3300 (N$\left.\mathrm{H}_{\text {stretch }}\right), 3024,2941,2865\left(\mathrm{C}-\mathrm{H}_{\text {stretch }}\right), 1646\left(\mathrm{C}=\mathrm{O}_{\text {stretch }}\right) ; \delta_{\mathrm{H}}\left(400 \mathrm{MHz}, \mathrm{CDCl}_{3}\right)$ 7.37-7.22 $(5 \mathrm{H}, \mathrm{m}, \mathrm{H}-10, \mathrm{H}-11, \mathrm{H}-$ 12), $5.97(1 \mathrm{H}$, br s, NH), $5.61(1 \mathrm{H}$, br d, J $10.0 \mathrm{~Hz}, \mathrm{H}-5), 5.54(1 \mathrm{H}, \mathrm{br} \mathrm{d}, J 10.0 \mathrm{~Hz}, \mathrm{H}-6), 4.49(1 \mathrm{H}, \mathrm{d}, J 12.0 \mathrm{~Hz}$, H-8a), $4.44\left(1 \mathrm{H}, \mathrm{d}, J 12.0 \mathrm{~Hz}, \mathrm{H}-8_{b}\right), 4.42-4.35(1 \mathrm{H}, \mathrm{m}, \mathrm{H}-21), 4.12\left(1 \mathrm{H}, \mathrm{dd}, J\right.$ 8.5, $\left.6.0 \mathrm{~Hz}, \mathrm{H}-\mathrm{2}_{a}\right), 3.71-3.54(3$ $\left.\mathrm{H}, \mathrm{m}, \mathrm{H}-7_{a}, \mathrm{H}-13_{a}, \mathrm{H}-24_{b}\right), 3.46\left(1 \mathrm{H}, \mathrm{d}, J 10.0 \mathrm{~Hz}, \mathrm{H}-13_{b}\right), 3.39\left(1 \mathrm{H}, \mathrm{dd}, J\right.$ 9.0, $\left.9.0 \mathrm{~Hz}, \mathrm{H}-7_{b}\right), 3.21(2 \mathrm{H}, \mathrm{br} \mathrm{s}, \mathrm{H}-$ 18), $2.47\left(1 \mathrm{H}, \mathrm{dd}, J 15.0,7.5 \mathrm{~Hz}, \mathrm{H}-20_{a}\right), 2.40$ ( $\left.1 \mathrm{H}, \mathrm{dd}, J 15.0,5.0 \mathrm{~Hz}, \mathrm{H}-2 \mathrm{O}_{b}\right), 2.28\left(1 \mathrm{H}, \mathrm{br} \mathrm{d}, J 18.0 \mathrm{~Hz}, \mathrm{H}-4_{a}\right)$, 2.07-1.91 (2 H, m, H-3, H-4 $\left.)_{b}\right), 1.87\left(1 \mathrm{H}, \mathrm{br}\right.$ d, J $\left.18.0 \mathrm{~Hz}, \mathrm{H}-1_{a}\right), 1.75\left(1 \mathrm{H}, \mathrm{br} \mathrm{d}, J 18.0 \mathrm{~Hz}, \mathrm{H}-1_{b}\right), 1.49(4 \mathrm{H}$, br s, $\mathrm{H}-16, \mathrm{H}-17), 1.43(3 \mathrm{H}, \mathrm{s}, \mathrm{H}-23), 1.36\left(3 \mathrm{H}, \mathrm{s}, \mathrm{H}-23\right.$ '), 1.08-0.96 (21 H, m, H-14, H-15); $\delta_{\mathrm{C}}\left(100 \mathrm{MHz}, \mathrm{CDCl}_{3}\right)$ 169.6 (C-19), 138.7 (C-Ar ${ }_{\text {quat }}$ ), 128.3 (C-Ar), 127.5 (C-Ar), 127.4 (C-Ar), 125.5 (C-5), 125.0 (C-6), 109.3 (C-22), 73.0 (C-8), 72.5 (C-21), 71.1 (C-7), 69.1 (C-24), 66.5 (C-13), 40.6 (C-20), 40.3 (C-18), 38.5 (C-2), 38.2 (C-3), 31.9 (C-1, C-16/C-17), 26.9 (C-23), 26.5 (C-4), 25.5 (C-23'), 23.1 (C-17/C-16), 18.1 (C-15), 11.9 (C-14); m/z (Cl) 588 (32) $[\mathrm{MH}]^{+}$; [HRMS (Cl) calcd. For $\mathrm{C}_{34} \mathrm{H}_{58} \mathrm{NO}_{5} \mathrm{Si}, 588.4084$. Found: [MH] $]^{+}, 588.4083$ (0.1 ppm error)].

N-[3-((1R,6S)-6-Benzyloxymethyl-1-hydroxymethyl-cyclohex-3-enyl)-propyl]-2-((S)-2,2-dimethyl-1,3-dioxolan-4yl)-acetamide (+)-19.

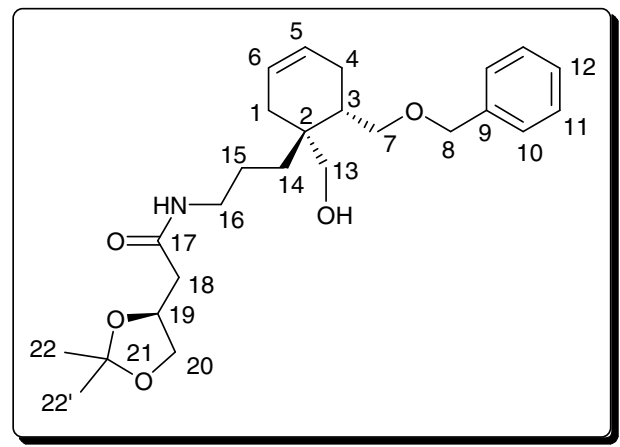


To a stirred solution of triisopropylsilyl ether $(+)-18(7.35 \mathrm{~g}, 12.5 \mathrm{mmol})$ in THF (100 mL) was added tetra- $n$ butylammonium fluoride ( $1 \mathrm{M}$ in THF, $20.0 \mathrm{~mL}, 20.0 \mathrm{mmol}$ ) at $0{ }^{\circ} \mathrm{C}$. The resulting solution was stirred at $\mathrm{rt}$ for $5 \mathrm{~h}$. The reaction was quenched by the careful addition of water, then partitioned between $\mathrm{CH}_{2} \mathrm{Cl}_{2}(100 \mathrm{~mL})$ and brine $(100 \mathrm{~mL})$ and the aqueous phase was extracted with $\mathrm{CH}_{2} \mathrm{Cl}_{2}(3 \times 50 \mathrm{~mL})$. The combined organic layers were dried over $\mathrm{Na}_{2} \mathrm{SO}_{4}$ and concentrated under reduced pressure to give a brown oil that was purified by column chromatography (eluting with 50-90\% EtOAc/iso-hexane) to furnish the alcohol (+)-19 as a colourless oil (5.38 g, $100 \%) ; R_{\mathrm{f}} 0.18$ (EtOAc); $[\alpha]_{D}+11.5$ (c 1.1, $\left.\mathrm{CHCl}_{3}\right) ; \mathrm{IR}$ (neat) $3314\left(\mathrm{O}-\mathrm{H}_{\text {stretch }}\right), 2984,2932\left(\mathrm{C}-\mathrm{H}_{\text {stretch }}\right), 1650$ $\left(\mathrm{C}=\mathrm{O}_{\text {stretch }}\right) ; \delta_{\mathrm{H}}\left(400 \mathrm{MHz}, \mathrm{CDCl}_{3}\right)$ 7.44-7.22 $(5 \mathrm{H}, \mathrm{m}, \mathrm{H}-10, \mathrm{H}-11, \mathrm{H}-12), 6.32(1 \mathrm{H}, \mathrm{br} \mathrm{s}, \mathrm{NH}), 5.58(1 \mathrm{H}, \mathrm{br} \mathrm{d}, \mathrm{J}$ $10.0 \mathrm{~Hz}, \mathrm{H}-5), 5.49(1 \mathrm{H}, \mathrm{br} \mathrm{d}, J 10.0 \mathrm{~Hz}, \mathrm{H}-6), 4.52\left(1 \mathrm{H}, \mathrm{d}, J 12.0 \mathrm{~Hz}, \mathrm{H}-8_{a}\right), 4.48\left(1 \mathrm{H}, \mathrm{d}, J 12.0 \mathrm{~Hz}, \mathrm{H}-8_{b}\right), 4.42-$ $4.36(1 \mathrm{H}, \mathrm{m}, \mathrm{H}-19), 4.11\left(1 \mathrm{H}, \mathrm{dd}, J\right.$ 8.5, $\left.6.0 \mathrm{~Hz}, \mathrm{H}-20_{a}\right), 3.71-3.55\left(3 \mathrm{H}, \mathrm{m}, \mathrm{H}-7_{a}, \mathrm{H}-13_{a}, \mathrm{H}-20_{b}\right), 3.51(1 \mathrm{H}, \mathrm{dd}, J$ 9.5, 5.0 Hz, H-7 $)$ ), 3.40-3.09 (3 H, m, H- 13b, H-16), 2.47 (1 H, dd, J 15.0, $\left.7.5 \mathrm{~Hz}, \mathrm{H}-18_{a}\right), 2.39(1 \mathrm{H}, \mathrm{dd}, J 15.0$, $\left.5.5 \mathrm{~Hz}, \mathrm{H}-18_{b}\right), 2.21\left(1 \mathrm{H}\right.$, ddd, J 18.5, 5.5, 3.0 Hz, H-4a), $2.07\left(1 \mathrm{H}, \mathrm{br} \mathrm{d}, J 18.5 \mathrm{~Hz}, \mathrm{H}-4_{b}\right), 1.87-1.62(4 \mathrm{H}, \mathrm{m}, \mathrm{H}-1$, $\mathrm{H}-3, \mathrm{H}-14_{a}$ ) $), 1.51$ (1 H, dd, J 14.0, 7.0 Hz, H-15a), 1.48 (1 H, dd, J 14.0, 7.0 Hz, H-15b), 1.43 (3 H, s, H-22), 1.36 (3 H, s, H-22'), $1.26\left(2 \mathrm{H}, \mathrm{m}, \mathrm{H}-14_{b}\right)$; $\delta_{\mathrm{C}}\left(100 \mathrm{MHz}, \mathrm{CDCl}_{3}\right) 169.9$ (C-17), $137.2\left(\mathrm{C}-\mathrm{Ar}_{\text {quat }}\right.$ ), 128.5 (C-Ar), 128.0 (CAr), 127.8 (C-Ar), 125.5 (C-5), 124.4 (C-6), 109.3 (C-21), 73.6 (C-8), 72.6 (C-19), 70.8 (C-13), 69.1 (C-20), 65.9 (C-7), 40.6 (C-18), 40.2 (C-16), 39.7 (C-3), 38.6 (C-2), 33.1 (C-1), 32.1 (C-14), 26.9 (C-22), 26.6 (C-4), 25.5 (C22'), 22.9 (C-15); m/z (Cl) 432 (100) [MH] ; HRMS (Cl) calcd. For $\mathrm{C}_{25} \mathrm{H}_{38} \mathrm{NO}_{5}, 432.2753$. Found: $[\mathrm{MH}]^{+}, 432.2750$ (0.7 ppm error)].

N-[3-((1R,6S)-6-Benzyloxymethyl-1-formyl-cyclohex-3-enyl)-propyl]-2-((S)-2,2-dimethyl-1,3-dioxolan-4-yl)acetamide (+)-20.

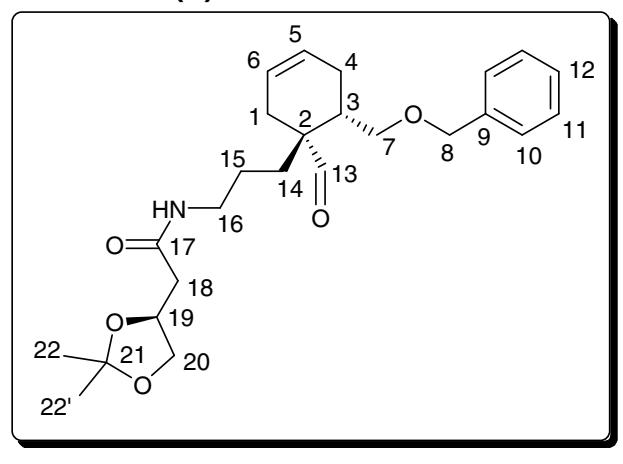

To a stirred solution of alcohol (+)-19 (5.56 g, $12.9 \mathrm{mmol})$ in $\mathrm{CH}_{2} \mathrm{Cl}_{2}(50 \mathrm{~mL})$ over $4 \AA$ molecular sieves was added $\mathrm{N}$-methylmorpholine- $N$-oxide $(1.81 \mathrm{~g}, 15.5 \mathrm{mmol})$ at rt. After stirring for $15 \mathrm{~min}$, tetra- $n$-propylammonium perruthenate $(340 \mathrm{mg}, 0.97 \mathrm{mmol}$ ) was added and the resulting mixture was stirred at $\mathrm{rt}$ for $30 \mathrm{~min}$. After evaporating the solvent, the crude product was purified by column chromatography (eluting with $60 \% \mathrm{EtOAc/iso-}$ hexane) to afford the aldehyde (+)-20 as a colourless oil $(5.48 \mathrm{~g}, 99 \%) ; R_{\mathrm{f}} 0.37$ (EtOAc); $[\alpha]_{D}+23.3$ (c 1.2, $\left.\mathrm{CHCl}_{3}\right)$; IR (neat) $3304\left(\mathrm{~N}-\mathrm{H}_{\text {stretch }}\right), 2961,2930,2863\left(\mathrm{C}-\mathrm{H}_{\text {stretch }}\right), 1718\left(\mathrm{C}=\mathrm{O}_{\text {aldehyde }}\right), 1647\left(\mathrm{C}=\mathrm{O}_{\text {amide }}\right) ; \delta_{\mathrm{H}}\left(400 \mathrm{MHz}, \mathrm{CDCl}_{3}\right)$ $9.57(1 \mathrm{H}, \mathrm{s}, \mathrm{H}-13), 7.45-7.18(5 \mathrm{H}, \mathrm{m}, \mathrm{H}-10, \mathrm{H}-11, \mathrm{H}-12), 6.07(1 \mathrm{H}, \mathrm{br} \mathrm{s}, \mathrm{NH}), 5.62(2 \mathrm{H}, \mathrm{m}, \mathrm{H}-5, \mathrm{H}-6), 4.44(1 \mathrm{H}$, d, J $\left.12.0 \mathrm{~Hz}, \mathrm{H}-8_{a}\right), 4.39\left(1 \mathrm{H}, \mathrm{d}, J 12.0 \mathrm{~Hz}, \mathrm{H}-8_{b}\right), 4.39-4.36(1 \mathrm{H}, \mathrm{m}, \mathrm{H}-19), 4.12\left(1 \mathrm{H}, \mathrm{dd} J 8.5,6.0 \mathrm{~Hz}, \mathrm{H}-20_{a}\right)$, $3.60\left(1 \mathrm{H}, \mathrm{dd}, J\right.$ 8.5, $\left.7.0 \mathrm{~Hz}, \mathrm{H}-20_{b}\right), 3.40(2 \mathrm{H}, \mathrm{m}, \mathrm{H}-7), 3.20(2 \mathrm{H}, \mathrm{dd}, J$ 13.0, $7.0 \mathrm{~Hz}, \mathrm{H}-16), 2.47$ (1 H, dd, J 15.0, $\left.7.5 \mathrm{~Hz}, \mathrm{H}-18_{a}\right), 2.41\left(1 \mathrm{H}, \mathrm{dd}, J\right.$ 15.0, $\left.5.0 \mathrm{~Hz}, \mathrm{H}-18_{b}\right), 2.32-2.19\left(3 \mathrm{H}, \mathrm{m}, \mathrm{H}-1_{a}, \mathrm{H}-3, \mathrm{H}-4_{a}\right), 2.08-1.95\left(2 \mathrm{H}, \mathrm{m}, \mathrm{H}-1_{b}\right.$, $\left.\mathrm{H}-4_{b}\right), 1.58-1.53(2 \mathrm{H}, \mathrm{m}, \mathrm{H}-15), 1.50-1.43\left(1 \mathrm{H}, \mathrm{m}, \mathrm{H}-14_{a}\right), 1.42(3 \mathrm{H}, \mathrm{s}, \mathrm{H}-22), 1.36$ (3 H, s, H-22'), 1.32-1.29 (1 $\left.\mathrm{H}, \mathrm{m}, \mathrm{H}-14_{b}\right) ; \delta_{\mathrm{C}}\left(100 \mathrm{MHz}, \mathrm{CDCl}_{3}\right) 205.4$ (C-13), 169.9 (C-17), 137.9 (C-Ar quat), 128.4 (C-Ar), 128.3 (C-Ar), 127.62 (C-Ar), 127.59 (C-Ar), 125.0 (C-5), 123.9 (C-6), 109.4 (C-21), 73.2 (C-8), 72.5 (C-19), 70.7 (C-7), 69.1 (C- 
20), 40.6 (C-18), 39.9 (C-2), 39.6 (C-16), 38.3 (C-3), 29.8 (C-14), 26.9 (C-22), 26.4 (C-4), 26.3 (C-1), 25.5 (C-22'), 23.8 (C-15); m/z (Cl) 430 (17) [MH] $]^{+}$; HRMS (Cl) calcd. For $\mathrm{C}_{25} \mathrm{H}_{36} \mathrm{NO}_{45}, 430.2593$. Found: $[\mathrm{MH}]^{+}, 430.2591$ (0.5 ppm error)].

(1R,2'S,6S,9a'S)-6-(Benzyloxymethyl)-2'-(hydroxymethyl)-6', 7', 8', 9a'-tetrahydro-2'H-spiro[cyclohex[3]ene-1,9'pyrido[2, 1-b][1,3]oxazin]-4'(3'H)-one (-)-21.

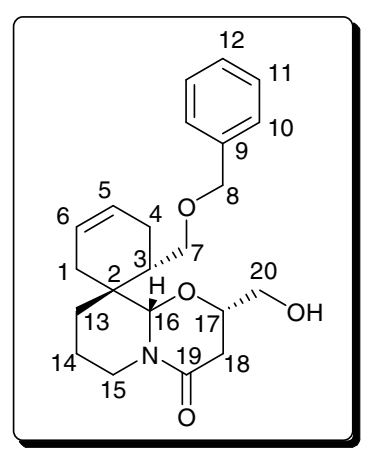

To a stirred solution of aldehyde (+)-20 $(5.30 \mathrm{~g}, 12.4 \mathrm{mmol})$ in $\mathrm{CH}_{2} \mathrm{Cl}_{2}$ (100 mL) was added tin(II) chloride dihydrate $(6.97 \mathrm{~g}, 30.9 \mathrm{mmol})$ at $\mathrm{rt}$. The resulting mixture was stirred at $\mathrm{rt}$ for $20 \mathrm{~h}$. The reaction mixture was then pre-absorbed onto silica followed by column chromatography (eluting with EtOAc) to afford the spirooxaquinolizidinone (-)-21 as a colourless oil (2.93 g, 78\%); $R_{\mathrm{f}} 0.11$ (EtOAc); $[\alpha]_{D}-102.0$ (c 1.0, $\mathrm{CHCl}_{3}$ ); IR (neat) $3393\left(\mathrm{O}-\mathrm{H}_{\text {stretch }}\right), 3025,2933,2856\left(\mathrm{C}-\mathrm{H}_{\text {stretch }}\right), 1634\left(\mathrm{C}=\mathrm{O}_{\text {amide }}\right) ; \delta_{\mathrm{H}}\left(400 \mathrm{MHz}, \mathrm{CDCl}_{3}\right)$ 7.40-7.25 $(5 \mathrm{H}, \mathrm{m}, \mathrm{H}-$ 10, H-11, H-12), 5.58-5.54 (2 H, m, H-5, H-6), $4.72(1 \mathrm{H}, \mathrm{s}, \mathrm{H}-16), 4.69-4.67\left(1 \mathrm{H}, \mathrm{m}, \mathrm{H}-15_{\mathrm{a}}\right), 4.53(1 \mathrm{H}, \mathrm{d}, \mathrm{J} 12.0$ $\left.\mathrm{Hz}, \mathrm{H}-8_{a}\right), 4.48\left(1 \mathrm{H}, \mathrm{d}, J 12.0 \mathrm{~Hz}, \mathrm{H}-8_{b}\right), 3.93\left(1 \mathrm{H}, \mathrm{dd}, J\right.$ 10.0, $\left.5.0 \mathrm{~Hz}, \mathrm{H}-7_{a}\right), 3.71(1 \mathrm{H}, \mathrm{dddd}, J$ 12.0, 6.0, 3.0, 3.0 $\mathrm{Hz}, \mathrm{H}-17), 3.62\left(1 \mathrm{H}, \mathrm{br} d, J 11.0 \mathrm{~Hz}, \mathrm{H}-20_{a}\right), 3.46-3.42\left(1 \mathrm{H}, \mathrm{m}, \mathrm{H}-20_{b}\right), 3.41\left(1 \mathrm{H}, \mathrm{dd}, J 10.0,6.5 \mathrm{~Hz}, \mathrm{H}-7_{b}\right), 2.51$ $\left(1 \mathrm{H}, \mathrm{d}, J 12.0 \mathrm{~Hz}, \mathrm{H}-18_{a}\right), 2.46\left(1 \mathrm{H}, \mathrm{d}, J 12.0 \mathrm{~Hz}, \mathrm{H}-18_{b}\right), 2.41-2.32\left(2 \mathrm{H}, \mathrm{m}, \mathrm{H}-14_{a}, \mathrm{H}-15_{b}\right), 2.26-2.21(3 \mathrm{H}, \mathrm{m}, \mathrm{H}-$ 4, OH), 1.81-1.71 (3 H, m, H-1 $\left., \mathrm{H}-3, \mathrm{H}-14_{b}\right), 1.56-1.46$ (3 H, m, H-1, $\mathrm{H}-13$ ); $\delta_{\mathrm{C}}\left(100 \mathrm{MHz}, \mathrm{CDCl}_{3}\right)$ 167.6, (C-19), 138.8 (C-Ar quat ), 128.8 (C-Ar), 128.1 (C-Ar), 128.0 (C-Ar), 126.1 (C-5), 124.9 (C-6), 91.3 (C-16), 73.0 (C-8), 72.9 (C-17), 71.4 (C-7), 64.4 (C-20), 41.9 (C-3), 40.0 (C-15), 38.7 (C-2), 34.3 (C-1), 33.6 (C-18), 27.8 (C-4), 26.2 (C14), 19.5 (C-13); m/z (Cl) $372(100)[\mathrm{MH}]^{+}$; HRMS (Cl) calcd. For $\mathrm{C}_{22} \mathrm{H}_{30} \mathrm{NO}_{4}, 372.2175$. Found: $[\mathrm{MH}]^{+}, 371.2173$ (0.5 ppm error)].

((1R,2'S,6S,9a'S)-6-(Benzyloxymethyl)-4'-oxo-3', 4',6', 7', 8', 9a'-hexahydro-2'H-spiro[cyclohex[3]ene-1,9'-pyrido[2, 1b][1,3]oxazine]-2'-yl)methyl anthracene-9-carboxylate (-)-22.

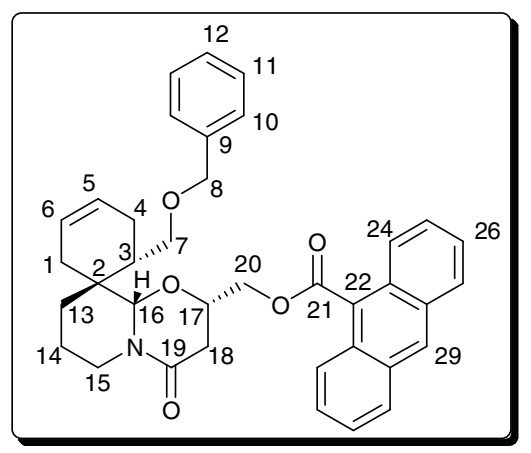

To a stirred solution of 9-anthracenecarboxylic acid $(90.0 \mathrm{mg}, 0.40 \mathrm{mmol})$ in $\mathrm{CH}_{2} \mathrm{Cl}_{2}$ at $0{ }^{\circ} \mathrm{C}$ was added a drop of DMF followed by oxalyl chloride $(71 \mu \mathrm{L}, 0.81 \mathrm{mmol})$. The solvent was removed in vacuo after $30 \mathrm{~min}$ and the 
resulting yellow solid dried under reduced pressure for $1 \mathrm{~h}$. It was then dissolved in $\mathrm{CH}_{2} \mathrm{Cl}_{2}(1.5 \mathrm{~mL})$ and added to a solution of alcohol (-)-21 $(75.0 \mathrm{mg}, 0.20 \mathrm{mmol})$ and pyridine $(82 \mu \mathrm{L}, 1.01 \mathrm{mmol})$ in $\mathrm{CH}_{2} \mathrm{Cl}_{2}(1.5 \mathrm{~mL})$. The final reaction mixture was stirred at $\mathrm{rt}$ for $57 \mathrm{~h}$. The reaction was then partitioned between more $\mathrm{CH}_{2} \mathrm{Cl}_{2}(10 \mathrm{~mL})$ and aqueous $\mathrm{NaHCO}_{3}$ (sat. $10 \mathrm{~mL}$ ). The organic layer was subsequently washed with aqueous $\mathrm{HCl}(10 \%, 10 \mathrm{~mL})$ and then dried over $\mathrm{Na}_{2} \mathrm{SO}_{4}$. The solvent was removed in vacuo and the crude solid purified by column chromatography (eluting with 30-100\% EtOAc/PE) to give the title compound (-)-22 as a dark brown solid that was re-crystallised from acetone to give yellow crystals (107 mg, 92\%); m.p. 93-95 ${ }^{\circ} \mathrm{C} ; R_{\mathrm{f}} 0.53(80 \% \mathrm{EtOAc} / \mathrm{PE})$; $[\alpha]_{D}-31.4$ (c 2.1, $\left.\mathrm{CHCl}_{3}\right)$; IR (neat) 3025, 2931, $2856\left(\mathrm{C}-\mathrm{H}_{\text {stretch }}\right), 1723\left(\mathrm{C}=\mathrm{O}_{\text {ester }}\right), 1634\left(\mathrm{C}=\mathrm{O}_{\text {amide }}\right) ; \delta_{\mathrm{H}}(400 \mathrm{MHz}$ $\left.\mathrm{CDCl}_{3}\right) 8.51(1 \mathrm{H}, \mathrm{s}, \mathrm{H}-29), 8.05(2 \mathrm{H}, \mathrm{d}, J 8.5 \mathrm{~Hz}, \mathrm{H}-24), 8.01(2 \mathrm{H}, J 8.5 \mathrm{~Hz}, \mathrm{H}-27), 7.58-7.47(4 \mathrm{H}, \mathrm{m}, \mathrm{H}-25, \mathrm{H}-$ 26), 7.21-7.20 (3 H, H-11, H-12), 7.00-6.94 (2 H, H-10), 5.57 (2 H, br s, H-5, H-6), 4.80 (1 H, dd, J 12.0, 3.0 Hz, $\left.\mathrm{H}-20_{a}\right)$, 4.74-4.70 (1 H, m, H-15a), $4.68(1 \mathrm{H}, \mathrm{s}, \mathrm{H}-16)$, $4.38\left(1 \mathrm{H}, \mathrm{dd}, J 12.0,6.5 \mathrm{~Hz}, \mathrm{H}-2 \mathrm{O}_{b}\right), 4.18-4.12(1 \mathrm{H}, \mathrm{m}, \mathrm{H}-$ 17), $3.90\left(1 \mathrm{H}, \mathrm{d}, J 12.0 \mathrm{~Hz}, \mathrm{H}-8_{a}\right), 3.87\left(1 \mathrm{H}, \mathrm{dd}, J\right.$ 9.5, $\left.4.0 \mathrm{~Hz}, \mathrm{H}-7_{a}\right), 3.86\left(1 \mathrm{H}, \mathrm{d}, J 12.0 \mathrm{~Hz}, \mathrm{H}-8_{b}\right), 3.26(1 \mathrm{H}$, dd, J 9.5, $\left.9.5 \mathrm{~Hz}, \mathrm{H}-7_{b}\right), 2.57\left(1 \mathrm{H}, \mathrm{d}, J 16.5 \mathrm{~Hz}, \mathrm{H}-18_{a}\right), 2.54\left(1 \mathrm{H}, \mathrm{d}, J 16.5 \mathrm{~Hz}, \mathrm{H}-18_{b}\right), 2.46-2.38\left(2 \mathrm{H}, \mathrm{m}, \mathrm{H}-4_{a}\right.$, $\left.\mathrm{H}-15_{b}\right), 2.30\left(1 \mathrm{H}, \mathrm{br} d, J 18.5 \mathrm{~Hz}, \mathrm{H}-14_{a}\right), 2.11-2.04\left(1 \mathrm{H}, \mathrm{m}, \mathrm{H}-4_{b}\right), 1.93\left(1 \mathrm{H}, \mathrm{br} \mathrm{d}, J 13.5 \mathrm{~Hz}, \mathrm{H}-1_{a}\right), 1.78-1.69$ (2 $\left.\mathrm{H}, \mathrm{m}, \mathrm{H}-3, \mathrm{H}-14_{b}\right), 1.59-1.46(2 \mathrm{H}, \mathrm{m}, \mathrm{H}-13), 1.36\left(1 \mathrm{H}, \mathrm{br} \mathrm{d}, \mathrm{J} 13.5 \mathrm{~Hz}, \mathrm{H}-1_{b}\right) ; \delta_{\mathrm{C}}\left(100 \mathrm{MHz}, \mathrm{CDCl}_{3}\right) 169.4(\mathrm{C}-21)$, 166.3 (C-19), 138.6 (C-Ar quat), 131.0 (C-Ar), 129.9 (C-Ar), 128.8 (C-Ar), 128.6 (C-Ar), 128.2 (C-Ar), 127.5 (C-Ar), 127.3 (C-Ar), 127.1 (C-Ar), 125.6 (C-Ar), 124.9 (C-Ar), 124.7 (C-5/C-6), 124.5 (C-6/C-5), 92.0 (C-16), 72.6 (C-8), 70.9 (C-17), 70.3 (C-7), 66.4 (C-20), 42.5 (C-3), 40.4 (C-15), 39.0 (C-2), 34.7 (C-18), 33.6 (C-1), 25.7, (C-4), 24.4 (C-14), 19.7 (C-13); m/z (Cl) 576 (100) [MH] ; HRMS (Cl) calcd. For $\mathrm{C}_{37} \mathrm{H}_{38} \mathrm{NO}_{5}, 576.2750$. Found: $[\mathrm{MH}]^{+}$, 576.2727 (3.9 ppm error)].

(1R,2'S,6S,9a'S)-6-(Benzyloxymethyl)-2'-((triisopropylsilyloxy)methyl)-6', 7', 8',9a'-tetrahydro-2'Hspiro[cyclohex[3]ene-1,9'-pyrido[2, 1-b][1,3]oxazin]-4'(3'H)-one (-)-23.

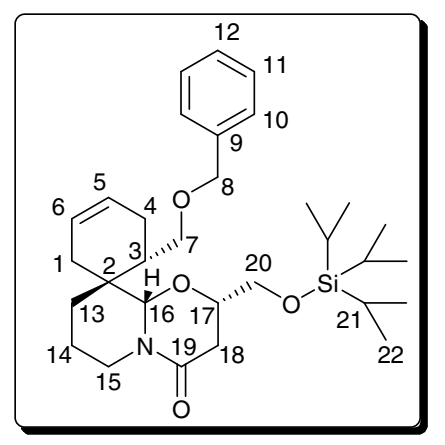

To a stirred solution of alcohol (-)-21 (750 mg, $2.02 \mathrm{mmol})$ in $\mathrm{CH}_{2} \mathrm{Cl}_{2}(20 \mathrm{~mL})$ at $0{ }^{\circ} \mathrm{C}$ was added 2,6-lutidine (1.20 $\mathrm{mL}, 10.1 \mathrm{mmol}$ ) followed by triisopropylsilyl trifluoromethanesulfonate $(1.36 \mathrm{~mL}, 5.05 \mathrm{mmol})$. The resulting solution was stirred at $\mathrm{rt}$ for $3 \mathrm{~h}$. The reaction mixture was then diluted with further $\mathrm{CH}_{2} \mathrm{Cl}_{2}(2 \mathrm{~mL})$ and partitioned between water $(25 \mathrm{~mL})$. The aqueous phase was extracted using $\mathrm{CH}_{2} \mathrm{Cl}_{2}(3 \times 15 \mathrm{~mL})$ and the combined organic layers dried over $\mathrm{Na}_{2} \mathrm{SO}_{4}$. The solvent was removed in vacuo and the crude product purified by column chromatography (eluting with 30-75\% EtOAc/PE) to furnish triisopropylsilyl ether (-)-23 as a colourless oil (1.04 g, 98\%); $R_{\mathrm{f}} 0.23$ (30\% EtOAc/PE); [ $\left.\alpha\right]_{D}-68.2$ (c 2.2, $\left.\mathrm{CHCl}_{3}\right)$; IR (neat) 3024, 2940, $2865\left(\mathrm{C}-\mathrm{H}_{\text {stretch }}\right), 1659\left(\mathrm{C}=\mathrm{O}_{\text {stretch }}\right)$; $\delta_{\mathrm{H}}\left(400 \mathrm{MHz}, \mathrm{CDCl}_{3}\right)$ 7.36-7.25 (5 H, m, H-10, H-11, H-12), 5.60-5.54 (2 H, m, H-5, H-6), 4.72 (1 H, dddd, J 13.0, 4.5, 2.0, $\left.2.0 \mathrm{~Hz}, \mathrm{H}-15_{a}\right), 4.61(1 \mathrm{H}, \mathrm{s}, \mathrm{H}-16), 4.49\left(1 \mathrm{H}, \mathrm{d}, J 12.0 \mathrm{~Hz}, \mathrm{H}-8_{a}\right), 4.46\left(1 \mathrm{H}, \mathrm{d}, J 12.0 \mathrm{~Hz}, \mathrm{H}-8_{b}\right), 4.02(1$ $\mathrm{H}$, dd, J 9.5, 4.0 Hz, H-7a $), 3.74-3.68\left(2 \mathrm{H}, \mathrm{m}, \mathrm{H}-17, \mathrm{H}-2 \mathrm{O}_{a}\right), 3.64-3.60\left(1 \mathrm{H}, \mathrm{m}, \mathrm{H}-2 \mathrm{O}_{b}\right), 3.37(1 \mathrm{H}, \mathrm{dd}, J 9.5,9.5$ 
$\left.\mathrm{Hz}, \mathrm{H}-7_{b}\right), 2.53-2.34\left(4 \mathrm{H}, \mathrm{m}, \mathrm{H}-14_{a}, \mathrm{H}-15_{b}, \mathrm{H}-18\right), 2.28\left(1 \mathrm{H}, \mathrm{br}\right.$ d, J $\left.18.5 \mathrm{~Hz}, \mathrm{H}-4_{a}\right), 2.15(1 \mathrm{H}, \mathrm{br} \mathrm{d}, J 18.5 \mathrm{~Hz}, \mathrm{H}-$ 14b), $1.92\left(1 \mathrm{H}, \mathrm{dd}, \mathrm{J}\right.$ 13.5, $\left.2.0 \mathrm{~Hz}, \mathrm{H}-1_{a}\right), 1.83-1.73\left(2 \mathrm{H}, \mathrm{m}, \mathrm{H}-3, \mathrm{H}-13_{a}\right), 1.60-1.49\left(2 \mathrm{H}, \mathrm{m}, \mathrm{H}-13_{b}, \mathrm{H}-4_{b}\right), 1.40(1$

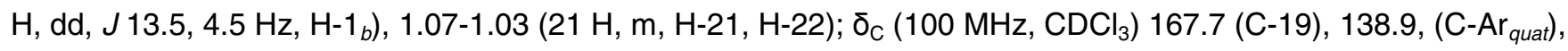
128.5, (C-Ar), 127.6 (C-Ar), 127.5 (C-Ar), 124.9 (C-5), 124.4 (C-6), 91.9 (C-16), 73.6 (C-17), 73.0 (C-8), 70.8 (C7), 65.2 (C-20), 42.5 (C-3), 40.3 (C-15), 39.0 (C-2), 34.9 (C-1), 33.9 (C-18), 25.8 (C-14), 24.7 (C-4), 19.8 (C-13), 18.1 (C-22), 12.0 (C-21); m/z (Cl) 528 (100) [MH] $]^{+}$; HRMS (Cl) calcd. For $\mathrm{C}_{31} \mathrm{H}_{50} \mathrm{NO}_{4} \mathrm{Si}, 528.3509$. Found: $[\mathrm{MH}]^{+}$, 528.3599 (1.9 ppm error)].

(1S,2S,2'S,6S,9a'S)-6-(Benzyloxymethyl)-2-hydroxy-2'-((triisopropylsilyloxy)methyl)-6', 7', 8', 9a'-tetrahydro-2'Hspiro[cyclohex[3]ene-1,9'-pyrido[2, 1-b][1,3]oxazin]-4'(3'H)-one (-)-24.

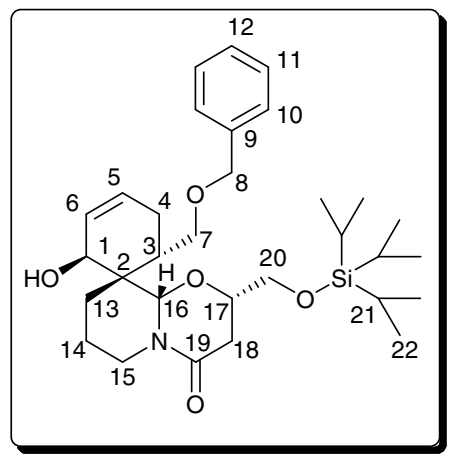

To a stirred solution of cyclohexene (-)-23 (100.0 mg, $0.19 \mathrm{mmol})$ in EtOH (3 mL) at rt was added selenium(IV) oxide $(105.5 \mathrm{mg}, 0.95 \mathrm{mmol})$. The final mixture was stirred at reflux for $17 \mathrm{~h}$. After cooling, a 1:1 mixture of $\mathrm{Et}_{2} \mathrm{O} / \mathrm{PE}$ was added. The mixture was filtered through Celite ${ }^{\circledR}$, the solvent was then removed in vacuo and the crude residue purified by column chromatography (eluting with $2 \% \mathrm{MeOH} / \mathrm{CH}_{2} \mathrm{Cl}_{2}$ ) to give alcohol (-)-24 as a pale yellow oil (80.0 mg, 78\%); $R_{\mathrm{f}} 0.25\left(4 \% \mathrm{MeOH} / \mathrm{CH}_{2} \mathrm{Cl}_{2}\right)$; $[\alpha]_{D}-45.3$ (c 2.5, $\left.\mathrm{CHCl}_{3}\right)$; IR (neat) $3405\left(\mathrm{OH}_{\text {stretch }}\right), 3026$, 2942, 2866 (C- $\left.\mathrm{H}_{\text {stretch }}\right), 1637$ ( $\left.\mathrm{C}=\mathrm{O}_{\text {stretch }}\right), 1456$; $\delta_{\mathrm{H}}\left(400 \mathrm{MHz}, \mathrm{CD}_{3} \mathrm{OD}\right)$ 7.33-7.25 (5 H, m, H-10, H-11, H-12), 5.78$5.74(1 \mathrm{H}, \mathrm{m}, \mathrm{H}-5), 5.72-5.68(1 \mathrm{H}, \mathrm{m}, \mathrm{H}-6), 4.96(1 \mathrm{H}, \mathrm{s}, \mathrm{H}-16), 4.66\left(1 \mathrm{H}, \mathrm{ddd}, J\right.$ 13.5, 4.5, $\left.2.0 \mathrm{~Hz}, \mathrm{H}-15_{a}\right), 4.51$ $\left(1 \mathrm{H}, \mathrm{d}, J 12.0 \mathrm{~Hz}, \mathrm{H}-8_{a}\right), 4.48\left(1 \mathrm{H}, \mathrm{d}, J 12.0 \mathrm{~Hz}, \mathrm{H}-8_{b}\right), 4.12(1 \mathrm{H}, \mathrm{d}, J 3.5 \mathrm{~Hz}, \mathrm{H}-1), 3.82(1 \mathrm{H}, \mathrm{dd}, J 9.5,4.0 \mathrm{~Hz}$, $\mathrm{H}-7_{a}$ ), 3.72-3.62 (3 H, m, H-17, H-20), $3.57\left(1 \mathrm{H}, \mathrm{dd}, J\right.$ 9.5, $\left.7.5 \mathrm{~Hz}, \mathrm{H}-7_{b}\right), 2.58-2.46\left(2 \mathrm{H}, \mathrm{m}, \mathrm{H}-4_{a}, \mathrm{H}-15_{b}\right), 2.34(1$ $\mathrm{H}$, dd, J 17.0, $\left.11.0 \mathrm{~Hz}, \mathrm{H}-18_{a}\right), 2.25\left(1 \mathrm{H}, \mathrm{dd}, J 17.0,3.0 \mathrm{~Hz}, \mathrm{H}-18_{b}\right), 2.14(1 \mathrm{H}$, ddd, J 18.0, 5.0, 5.0 Hz, H-4b), $2.01\left(1 \mathrm{H}, \mathrm{dd}, J\right.$ 13.5, $\left.2.0 \mathrm{~Hz}, \mathrm{H}-13_{a}\right), 1.95-1.89(1 \mathrm{H}, \mathrm{m}, \mathrm{H}-3), 1.95-1.73\left(1 \mathrm{H}, \mathrm{m}, \mathrm{H}-14{ }_{a}\right), 1.64(1 \mathrm{H}, \mathrm{ddd}, \mathrm{J}, 13.5$, 13.0, $3.5 \mathrm{~Hz}, \mathrm{H}-13 b)$, 1.53-1.46 (1 H, m, H-14b), 1.11-1.05 (21 H, m, H-21, H-22); $\delta_{\mathrm{C}}\left(100 \mathrm{MHz}, \mathrm{CD}_{3} \mathrm{OD}\right) 170.7$ (C-19), 140.5 (C-9), 130.1 (C-5/C-6), 130.0 (C-6/C-5), 129.8 (C-Ar), 129.1 (C-Ar), 129.0 (C-Ar), 91.9 (C-16), 75.2 (C-17), 74.2 (C-8), 72.1 (C-7), 66.6 (C-20), 64.9 (C-1), 44.4 (C-2), 41.6 (C-15), 40.4 (C-3), 35.3 (C-18), 31.6 (C13), 29.6 (C-4), 21.5 (C-14), 18.4 (C-22), 13.0 (C-21); m/z (ESI) 544 (100) [MH] ${ }^{+}$; HRMS (ESI) calcd. For $\mathrm{C}_{31} \mathrm{H}_{50} \mathrm{NO}_{5} \mathrm{Si}, 544.3453$. Found: $[\mathrm{MH}]^{+}, 544.3445$ (1.5 ppm error)].

(1R,2'S,6S,9a'S)-6-(Benzyloxymethyl)-2'-((triisopropylsilyloxy)methyl)-6', 7', 8', 9a'-tetrahydro-2'Hspiro[cyclohex[3]ene-1,9'-pyrido[2,1-b][1,3]oxazine]-2,4'(3'H)-dione (-)-25. 


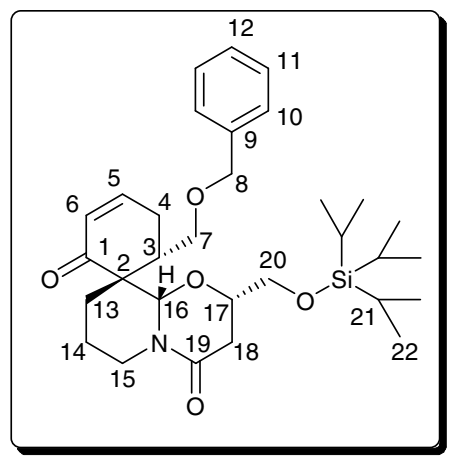

To a stirred solution of alcohol (-)-24 $(40.0 \mathrm{mg}, 0.07 \mathrm{mmol})$ in $\mathrm{CH}_{2} \mathrm{Cl}_{2}(2 \mathrm{~mL})$ at rt was added manganese(IV) oxide $(64.0 \mathrm{mg}, 0.73 \mathrm{mmol})$. The black suspension was stirred at reflux for $4 \mathrm{~h}$. The solids were filtered off and the filtrate purified by column chromatography (eluting with $1-2 \% \mathrm{MeOH} / \mathrm{CH}_{2} \mathrm{Cl}_{2}$ ) to give the enone (-)-25 (36.0 $\mathrm{mg}, 91 \%$ ) as a colourless oil; $R_{\mathrm{f}} 0.32\left(4 \% \mathrm{MeOH} / \mathrm{CH}_{2} \mathrm{Cl}_{2}\right)$; $[\alpha]_{D}-99.6$ (c 1.6, $\left.\mathrm{CHCl}_{3}\right)$; IR (neat) 2941, 2865, (C$\left.\mathrm{H}_{\text {stretch }}\right), 1660\left(\mathrm{C}=\mathrm{O}_{\text {stretch }}\right), 1460 ; \delta_{\mathrm{H}}\left(400 \mathrm{MHz}, \mathrm{CDCl}_{3}\right)$ 7.32-7.24 $(5 \mathrm{H}, \mathrm{m}, \mathrm{H}-10, \mathrm{H}-11, \mathrm{H}-12), 6.75(1 \mathrm{H}, \mathrm{ddd}, J$ 10.0, 5.0, 3.5 Hz, H-5), 5.84 (1 H, ddd, J 10.0, 2.0, $2.0 \mathrm{~Hz}, \mathrm{H}-6), 4.75$ (1 H, s, H-16), 4.64 (1 H, ddd, J 13.0, 4.3, $\left.4.5 \mathrm{~Hz}, \mathrm{H}-15_{a}\right), 4.45(2 \mathrm{H}, \mathrm{s}, \mathrm{H}-8), 3.80(1 \mathrm{H}, \mathrm{dd}, J$ 9.5, $4.5 \mathrm{~Hz}, \mathrm{H}-7 \mathrm{a}), 3.70-3.64(1 \mathrm{H}, \mathrm{m}, \mathrm{H}-17), 3.57(1 \mathrm{H}, \mathrm{dd}, J$ 10.5, $\left.6.0 \mathrm{~Hz}, \mathrm{H}-20_{a}\right), 3.52\left(1 \mathrm{H}, \mathrm{dd}, J 9.5,1.0 \mathrm{~Hz}, \mathrm{H}-7_{b}\right), 3.50\left(1 \mathrm{H}, \mathrm{dd}, J 10.5,4.5 \mathrm{~Hz}, \mathrm{H}-20_{b}\right), 2.76(1 \mathrm{H}, \mathrm{dddd}, J$ 19.0, 8.0, 3.5, $\left.2.0 \mathrm{~Hz}, \mathrm{H}-4_{a}\right)$, 2.45-2.43 (1 H, m, H-4b), 2.40-2.36 (1 H, m, H-15b), 2.26-2.25 (2 H, m, H-18), 2.17 (1 H, dddd, J 8.0, 8.0, 4.5, 4.5 Hz, H-3), 2.00 (1 H, 2.06-1.96 (1 H, m, H-14a), 1.91 (1 H, br d, J 14.5, Hz, H-13a), 1.73-1.69 (1 H, m, H-13 $)$, 1.47-1.40 (1 H, m, H-14b), 0.98-0.95 (21 H, m, H-21, H-22); $\delta_{\mathrm{C}}\left(100 \mathrm{MHz} \mathrm{CDCl}_{3}\right) 199.4$ (C-1), 166.7 (C-19), 147.8 (C-5), 138.5 (C-Ar $\left.{ }_{\text {quat }}\right), 130.5$ (C-6), 128.8 (C-Ar), 128.1 (C-Ar), 127.9 (C-Ar), 88.7 (C16), 74.1 (C-17), 73.4 (C-8), 70.4 (C-7), 65.3 (C-20), 49.7 (C-2), 44.1 (C-3), 39.7 (C-15), 34.5 (C-18), 32.3 (C-13), 27.4 (C-4), 20.6 (C-14), 17.8 (C-22), 11.6 (C-21); m/z (ESI) 542 (100) [MH] ; HRMS (ESI) calcd. For $\mathrm{C}_{31} \mathrm{H}_{48} \mathrm{NO}_{5} \mathrm{Si}, 542.3296$. Found: $[\mathrm{MH}]^{+}, 542.3291$ (1.0 ppm error)].

(1S,2R,2'S,6S,9a'S)-6-(Benzyloxymethyl)-2-hydroxy-2'-((triisopropylsilyloxy)methyl)-6', 7', 8',9a'-tetrahydro-2'Hspiro[cyclohex[3]ene-1, 9'-pyrido[2,1-b][1,3]oxazin]-4'(3'H)-one (-)-2a.

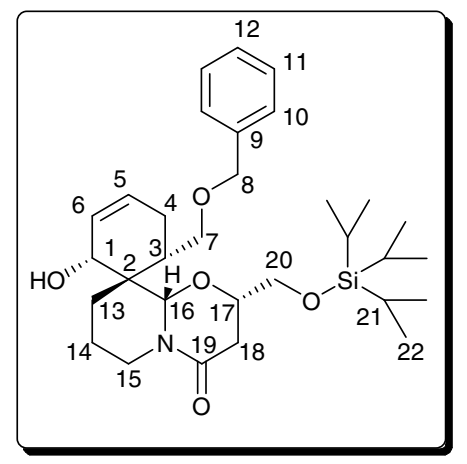

To a stirred solution of enone (-)-25 (36.0 mg, $0.07 \mathrm{mmol})$ in $\mathrm{MeOH}(2 \mathrm{~mL})$ at rt was added cerium(III) chloride heptahydrate $(26.0 \mathrm{mg}, 0.07 \mathrm{mmol})$. The solution was stirred at that temperature for $30 \mathrm{~min}$ before it was cooled to $0{ }^{\circ} \mathrm{C}$. To this was then added sodium borohydride $(5.0 \mathrm{mg}, 0.14 \mathrm{mmol})$. After stirring at $0{ }^{\circ} \mathrm{C}$ for $30 \mathrm{~min}$, the reaction was allowed to warm to $\mathrm{rt}$ at which it was stirred for a further $3 \mathrm{~h}$. The excess reducing agent was then quenched by the addition of acetone $(0.1 \mathrm{~mL})$. After removal of solvent, the crude residue was purified by column chromatography (eluting with $1-2 \% \mathrm{MeOH} / \mathrm{CH}_{2} \mathrm{Cl}_{2}$ ) to give alcohol (-)-2a as a colourless oil (26 mg, $72 \%$ ); $R_{\mathrm{f}} 0.34$ $\left(4 \% \mathrm{MeOH} / \mathrm{CH}_{2} \mathrm{Cl}_{2}\right)$; $[\alpha]_{D}-25.0$ (c 0.4, $\left.\mathrm{CHCl}_{3}\right)$; IR (neat) $3399\left(\mathrm{OH}_{\text {stretch }}\right), 2941,2866,\left(\mathrm{C}-\mathrm{H}_{\text {stretch }}\right), 1655\left(\mathrm{C}=\mathrm{O}_{\text {stretch }}\right)$, 
1458; $\delta_{\mathrm{H}}\left(400 \mathrm{MHz}, \mathrm{CD}_{3} \mathrm{OD}\right)$ 7.34-7.25 (5 H, m, H-10, H-11, H-12), 5.73-5.66 (2 H, m, H-5, H-6), $5.06(1 \mathrm{H}, \mathrm{s}, \mathrm{H}-$ 16), 4.58-4.52 (1 H, m, H-15a), $4.51\left(1 \mathrm{H}, \mathrm{d}, J 12.0 \mathrm{~Hz}, \mathrm{H}-8_{a}\right), 4.47\left(1 \mathrm{H}, \mathrm{d}, J 12.0 \mathrm{~Hz}, \mathrm{H}-8_{b}\right), 4.22(1 \mathrm{H}, \mathrm{d}, J 3.0$ $\mathrm{Hz}, \mathrm{H}-1), 4.08(1 \mathrm{H}, \mathrm{dd}, J$ 9.5, 3.0 Hz, H-7a), $3.83(1 \mathrm{H}$, ddd, J 14.0, 6.0, $2.5 \mathrm{~Hz}, \mathrm{H}-17), 3.78(1 \mathrm{H}, \mathrm{dd}, J 9.5,9.0$ $\left.\mathrm{Hz}, \mathrm{H}-7_{b}\right), 3.70\left(1 \mathrm{H}, \mathrm{d}, J 14.0 \mathrm{~Hz}, \mathrm{H}-20_{a}\right), 3.65\left(1 \mathrm{H}, \mathrm{d}, J 14.0 \mathrm{~Hz}, \mathrm{H}-2 \mathrm{O}_{b}\right), 2.73-2.64\left(2 \mathrm{H}, \mathrm{m}, \mathrm{H}-15_{b}, \mathrm{H}-18_{a}\right), 2.50$ $\left(1 \mathrm{H}, \mathrm{dd}, J\right.$ 18.0, $\left.4.0 \mathrm{~Hz} \mathrm{H}-4_{a}\right), 2.28\left(1 \mathrm{H}, \mathrm{dd}, J 16.5,3.0 \mathrm{~Hz}, \mathrm{H}-18_{b}\right), 2.25\left(1 \mathrm{H}, \mathrm{dd}, J\right.$ 18.0, $\left.6.5 \mathrm{~Hz}, \mathrm{H}-4_{b}\right), 1.92-1.88$ (2 H, m, H-3, H-13a), 1.75-1.66 (1 H, m, H-14a), 1.61-1.51 (2 H, m, H-13, $\left.\mathrm{H}-14_{b}\right), 1.10-1.06(21 \mathrm{H}, \mathrm{m}, \mathrm{H}-21, \mathrm{H}-$ 22); $\delta_{\mathrm{C}}\left(100 \mathrm{MHz}, \mathrm{CD}_{3} \mathrm{OD}\right) 170.6$ (C-19), 140.5 (C-Ar quat), 129.9 (C-Ar), 129.6 (C-Ar), 129.5 (C-Ar), 129.0 (C-5/C6), 128.7 (C-6/C-5), 93.8 (C-16), 75.7 (C-17), 73.8 (C-8), 72.6 (C-7), 70.1 (C-1), 65.8 (C-20), 43.4 (C-2), 42.9 (C3), 41.5 (C-15), 34.5 (C-18), 34.2 (C-13), 28.0 (C-4), 20.8 (C-14), 18.3 (C-22), 12.9 (C-21); m/z (ESI) 544 (100) $[\mathrm{MH}]^{+}$; HRMS (ESI) calcd. For $\mathrm{C}_{31} \mathrm{H}_{50} \mathrm{NO}_{5} \mathrm{Si}, 542.3453$. Found: [MH] $]^{+}, 544.3448$ (0.9 ppm error)].

(1) Von Langen, D. J.; Tolman, R. L. Tetrahedron: Asymmetry 1997, 8, 677.

(2) Reid, M.; Taylor, R. J. K. Tetrahedron Lett. 2004, 45, 4181.

(3) Jiménez, J. I.; Goetz, G.; Mau, C. M. S.; Yoshida, W. Y.; Scheuer, P. J.; Williamson, R. T.; Kelley, M. J. Org. Chem. 2000, 65, 8465. $1,793$.

(4) Danieli, B.; Leama, G.; Mauro, M.; Palmisano, G.; Passarella, D. Tetrahedron: Asymmetry 1990,

(5) O'Brien, P.; Tournayre, J. J. Tetrahedron 1997, 53, 17527. 


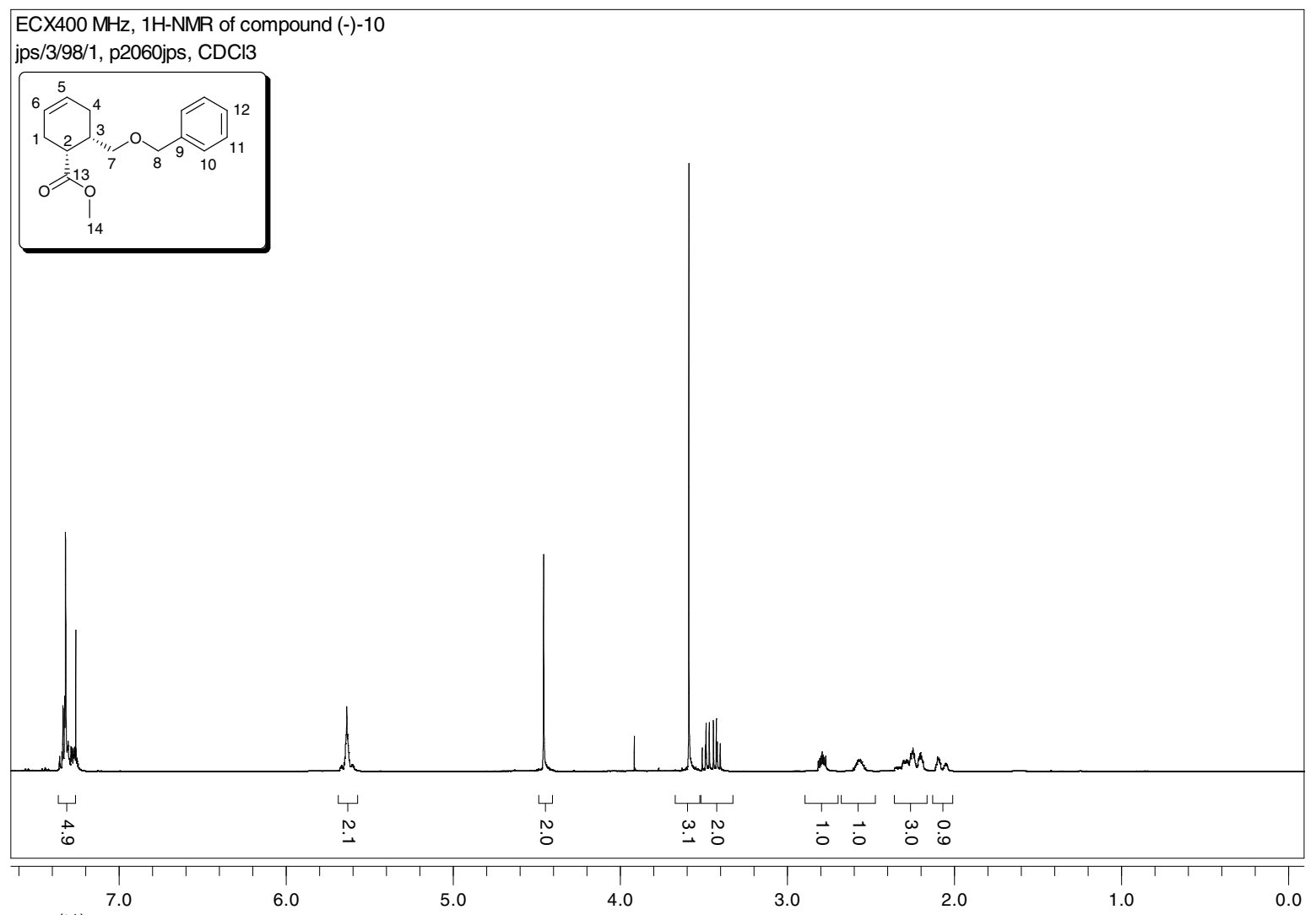

ppm (t1)
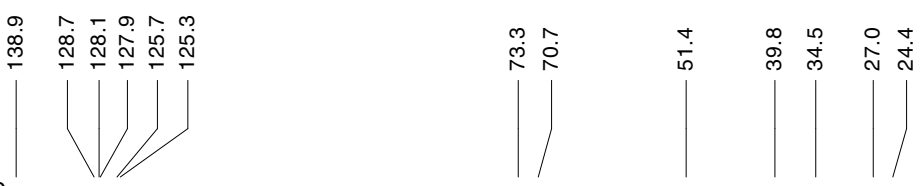

ECX400 MHz, 13C-NMR of compound (-)-10 jps/3/98/1, p2092jps, $\mathrm{CDCl} 3$
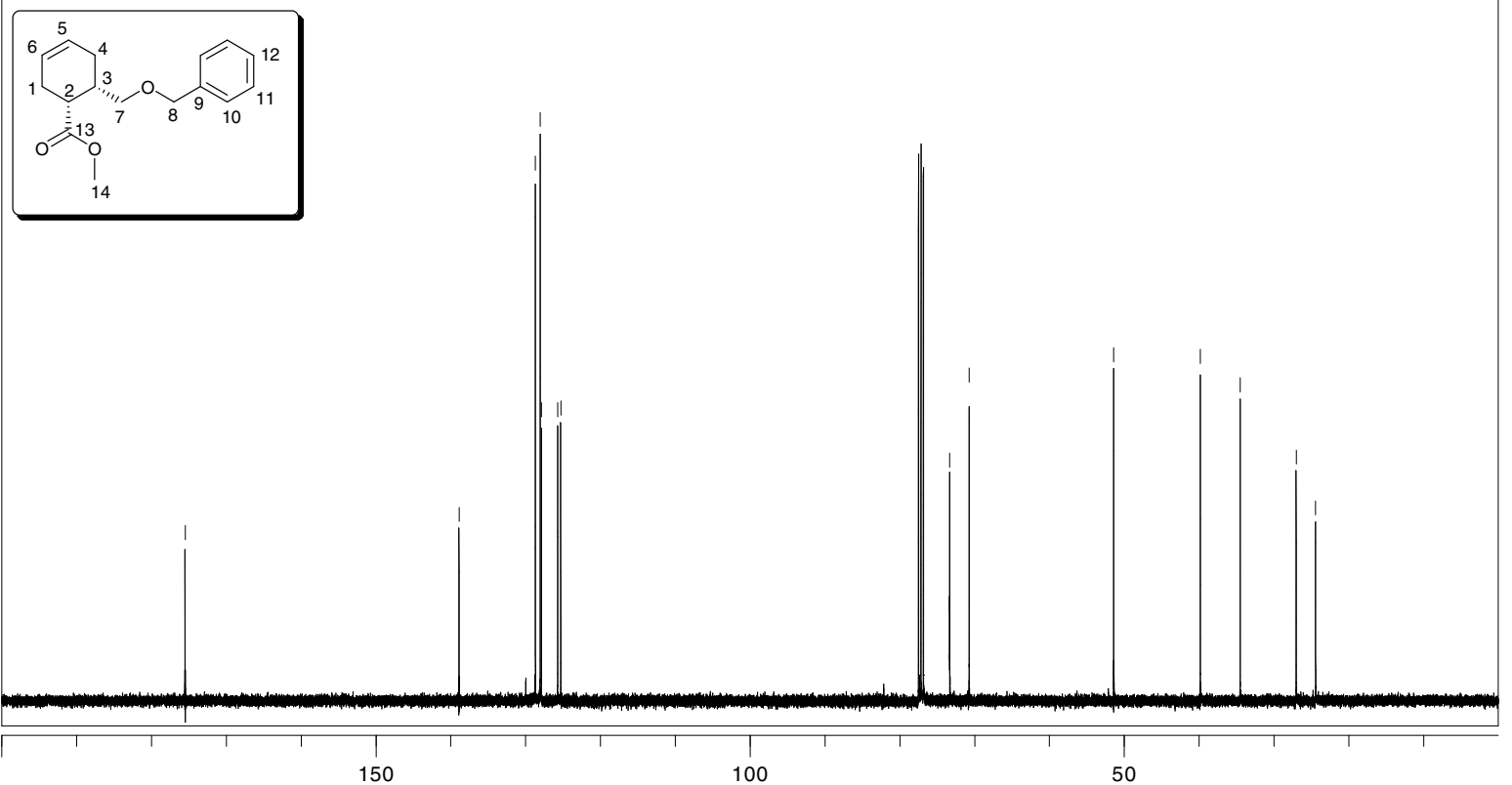

ppm (t1) 


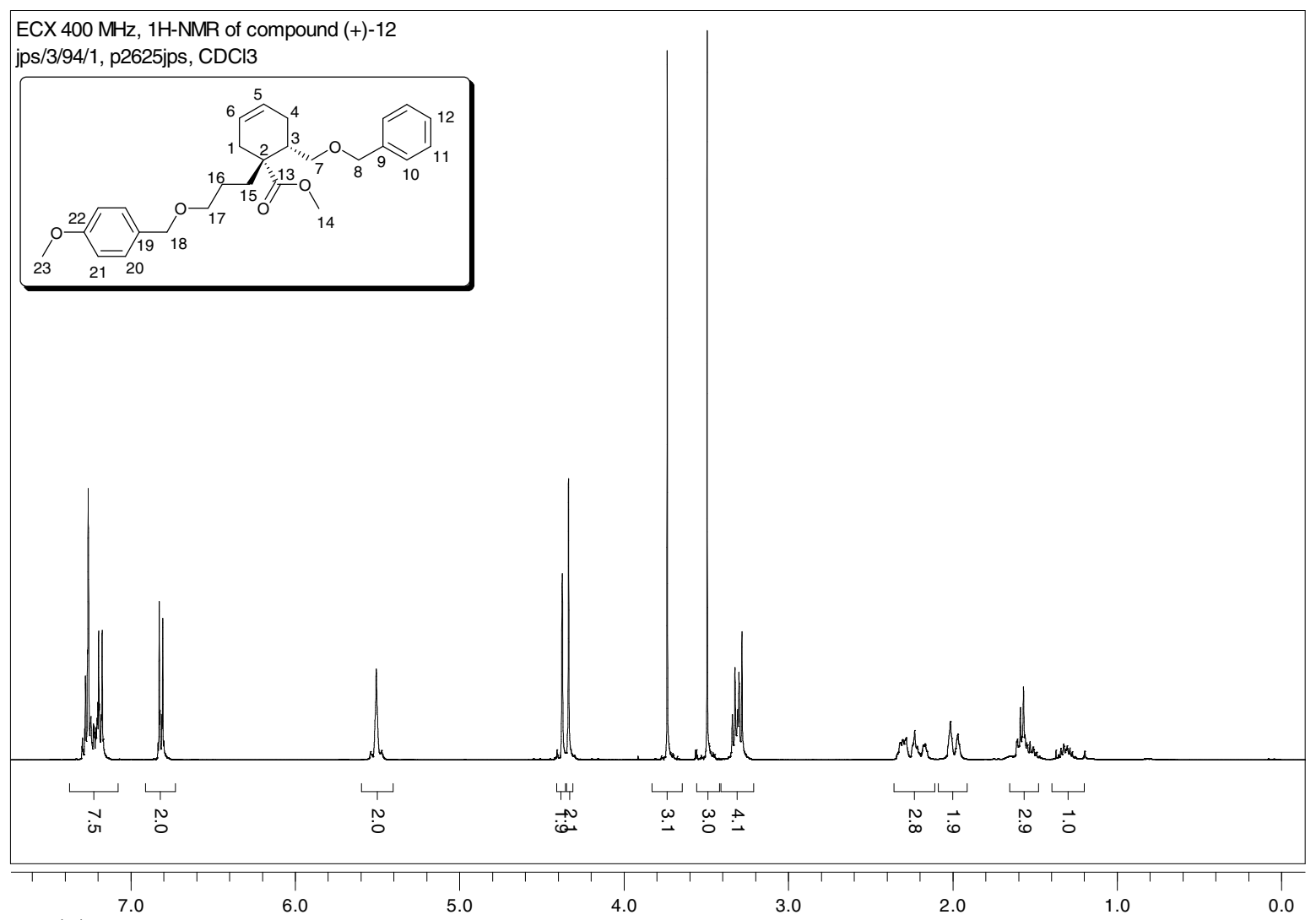

ppm (t1)

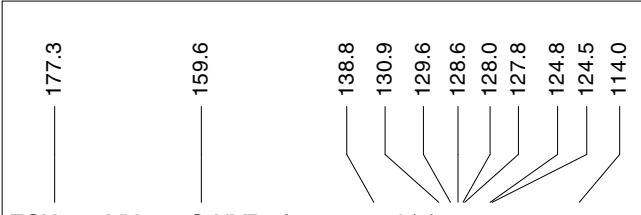

ECX $400 \mathrm{MHz}, 13 \mathrm{C}-\mathrm{NMR}$ of compound (+)-12 jps/3/94/1, p2651jps, CDCl3
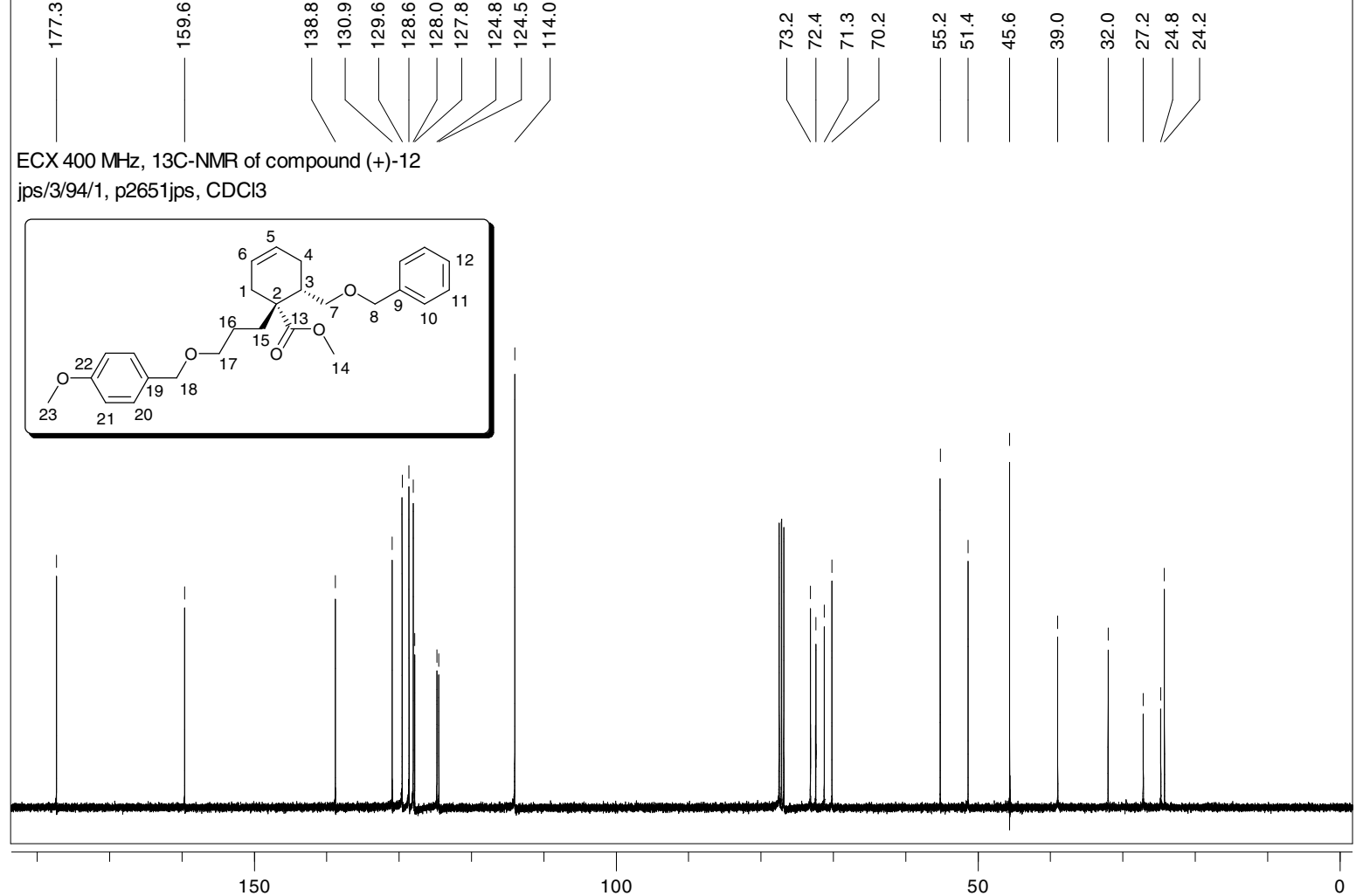

ppm (t1) 


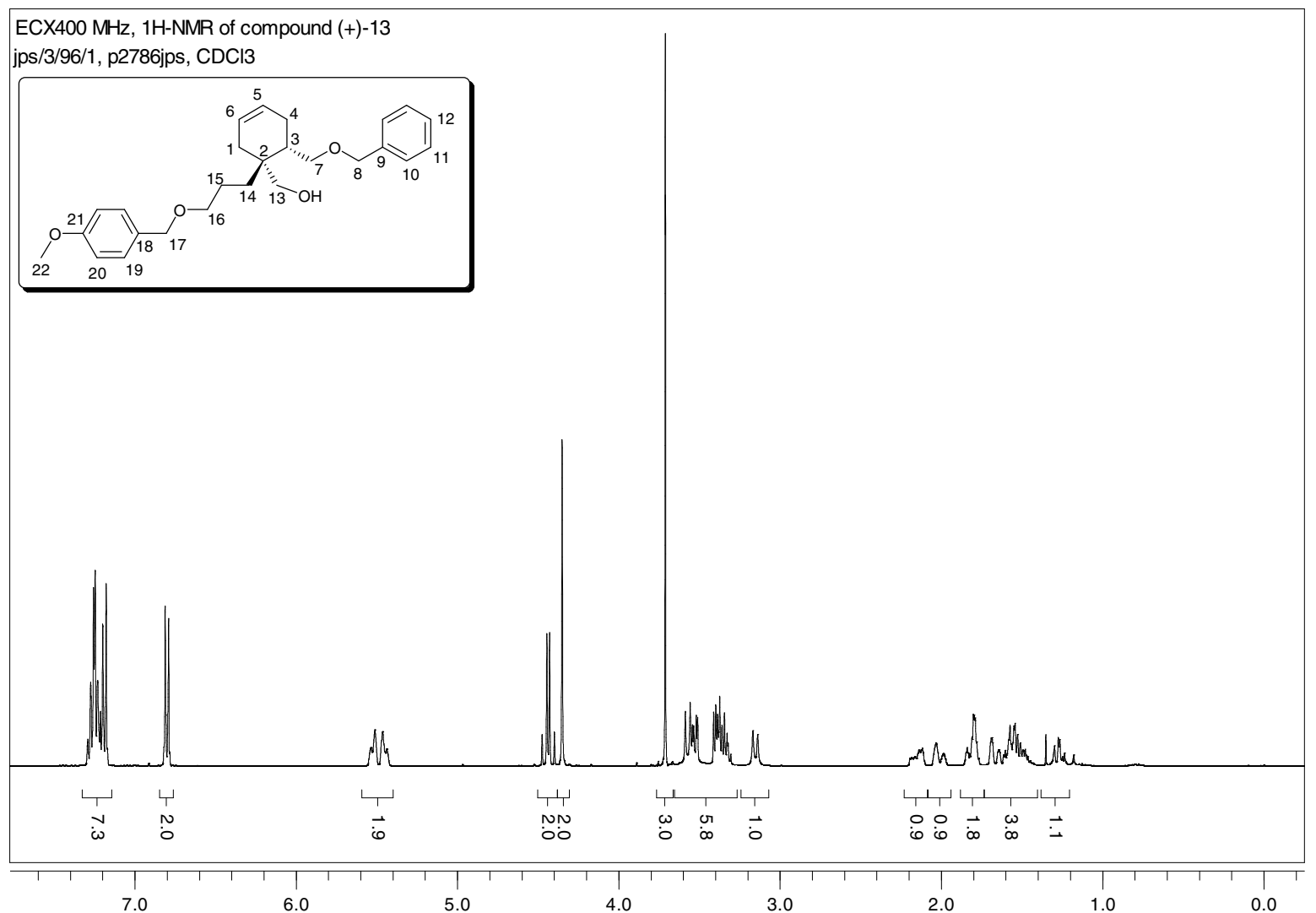

ppm (t1)

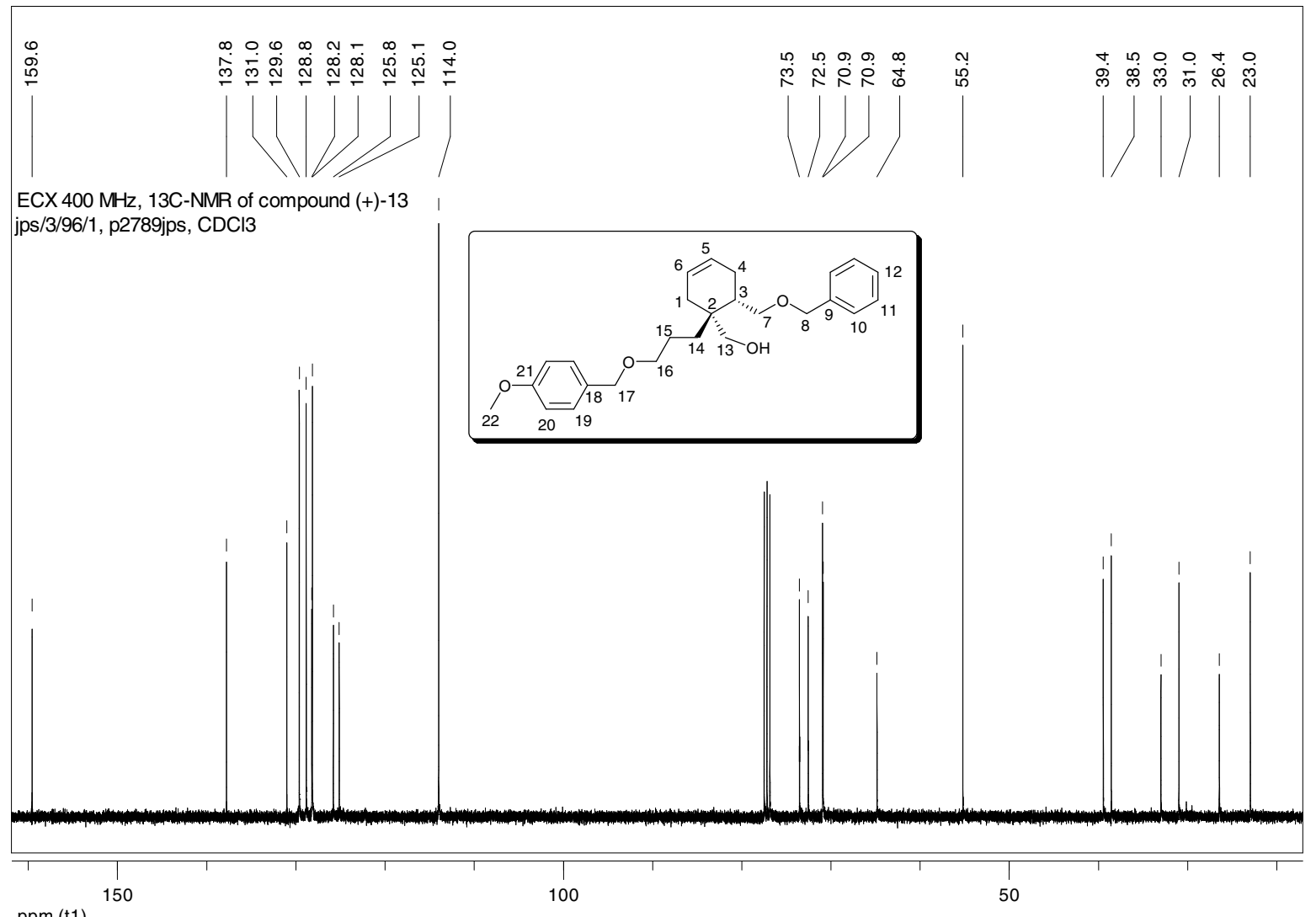

ppm (t1)

100 


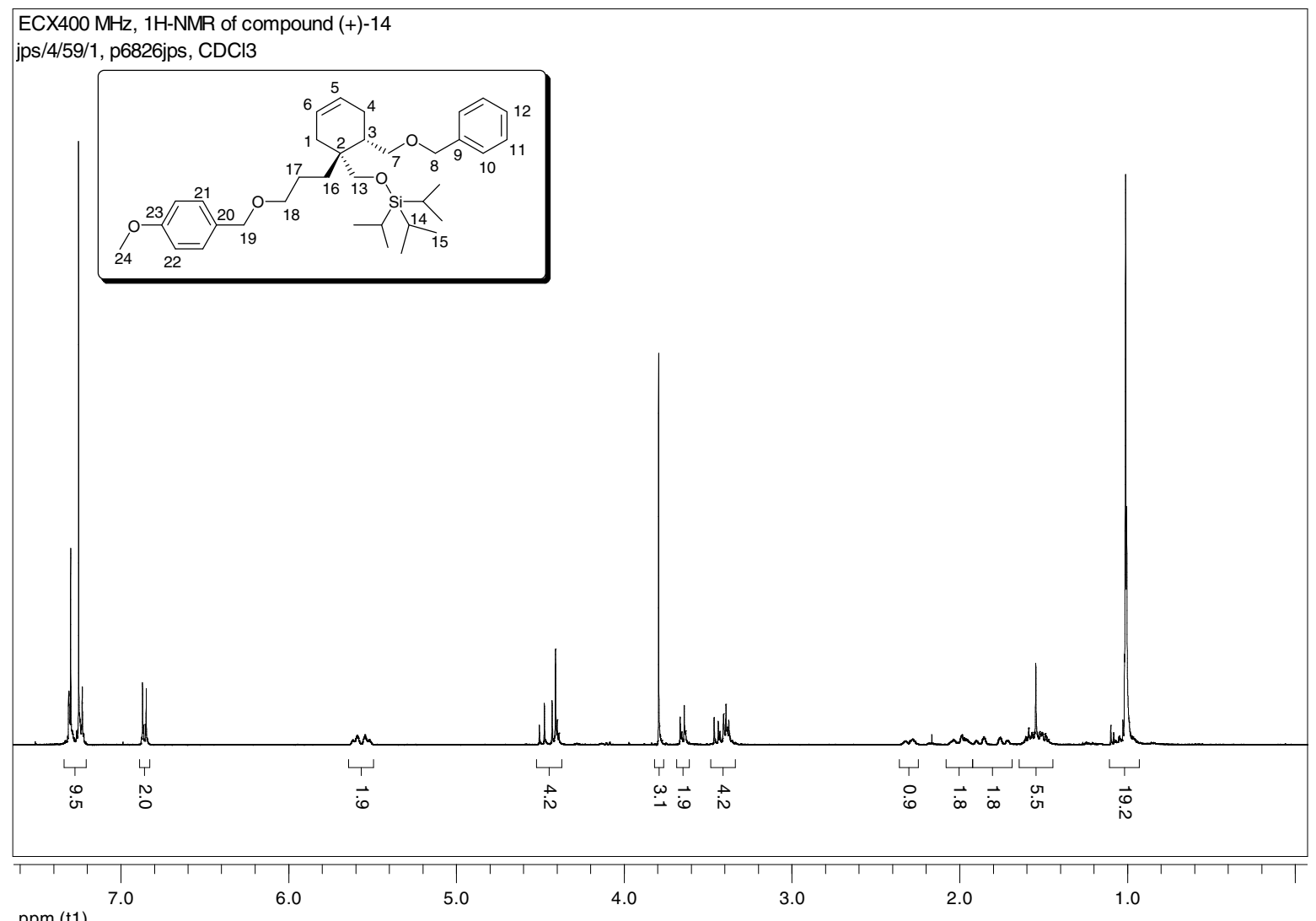

ppm (t1)

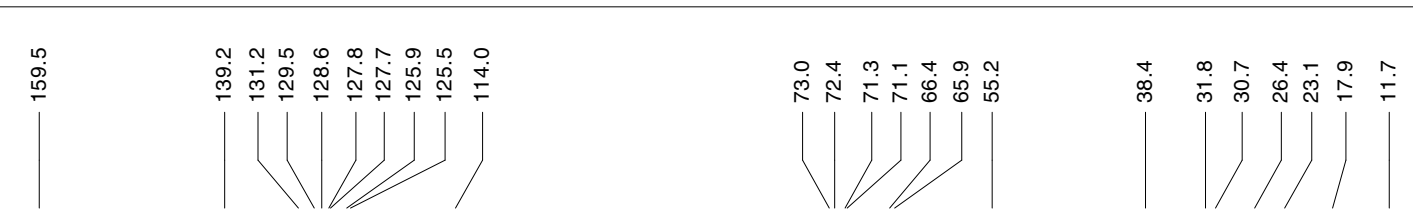

ECX400 MHz, 13C-NMR of compound (+)-14 jps/4/59/1, p6826jps, CDCl3
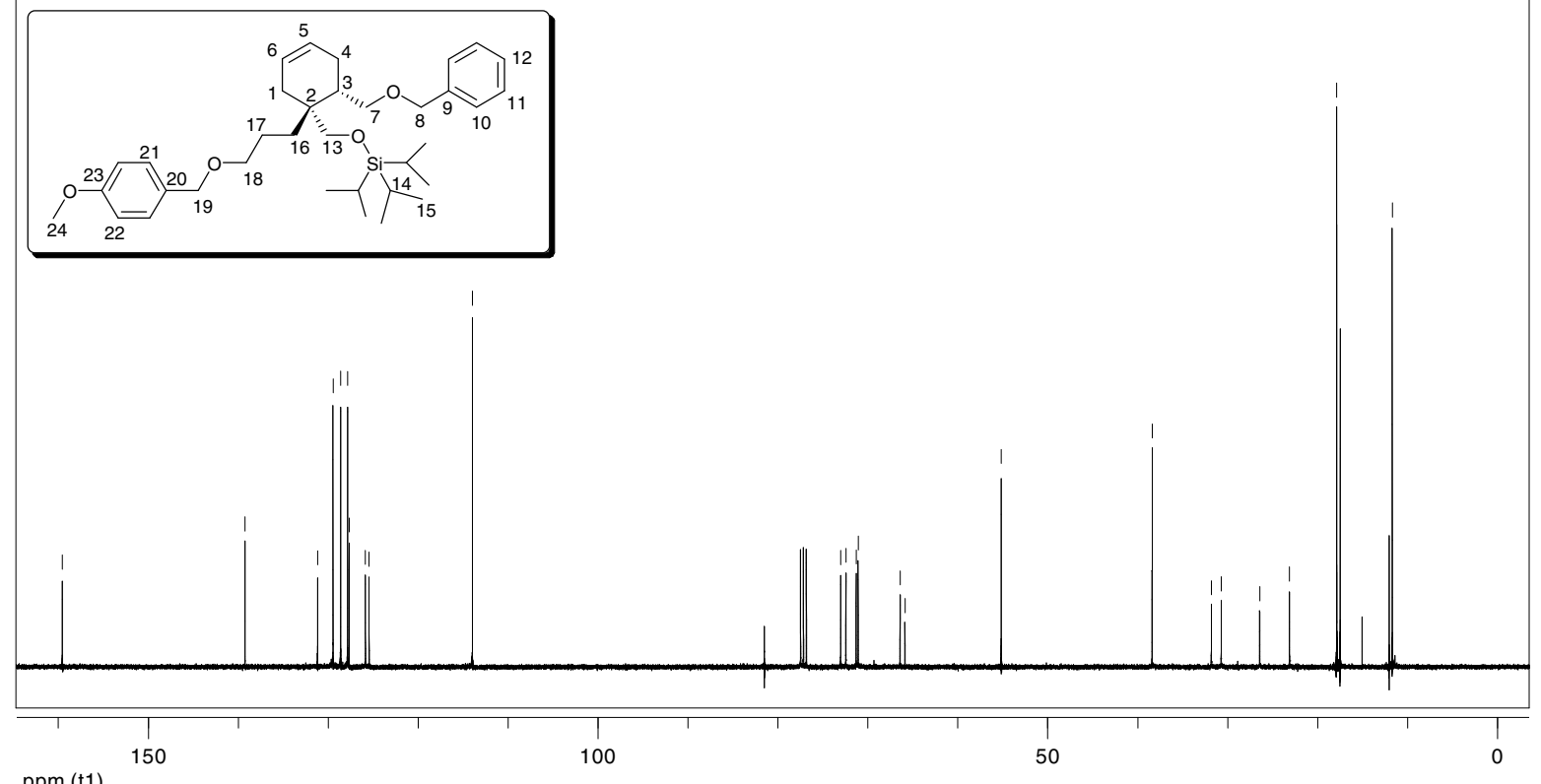


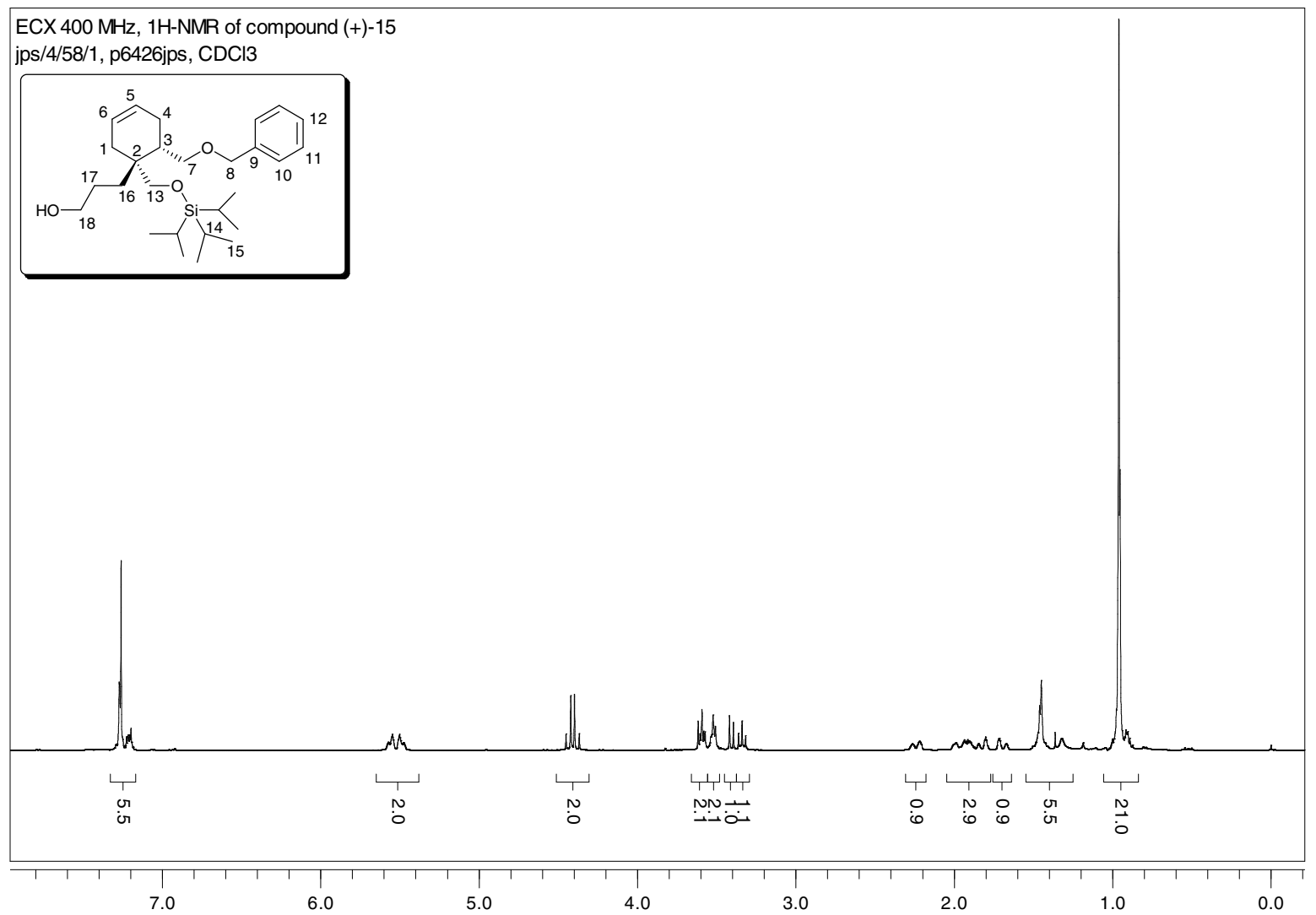

ppm (t1)
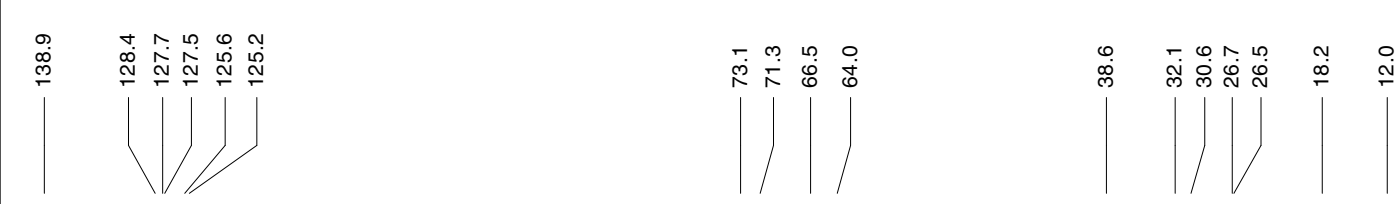

ECX400 MHz, 13C-NMR of compound (+)-15 jps/4/58/1, p6426jps, CDCl3
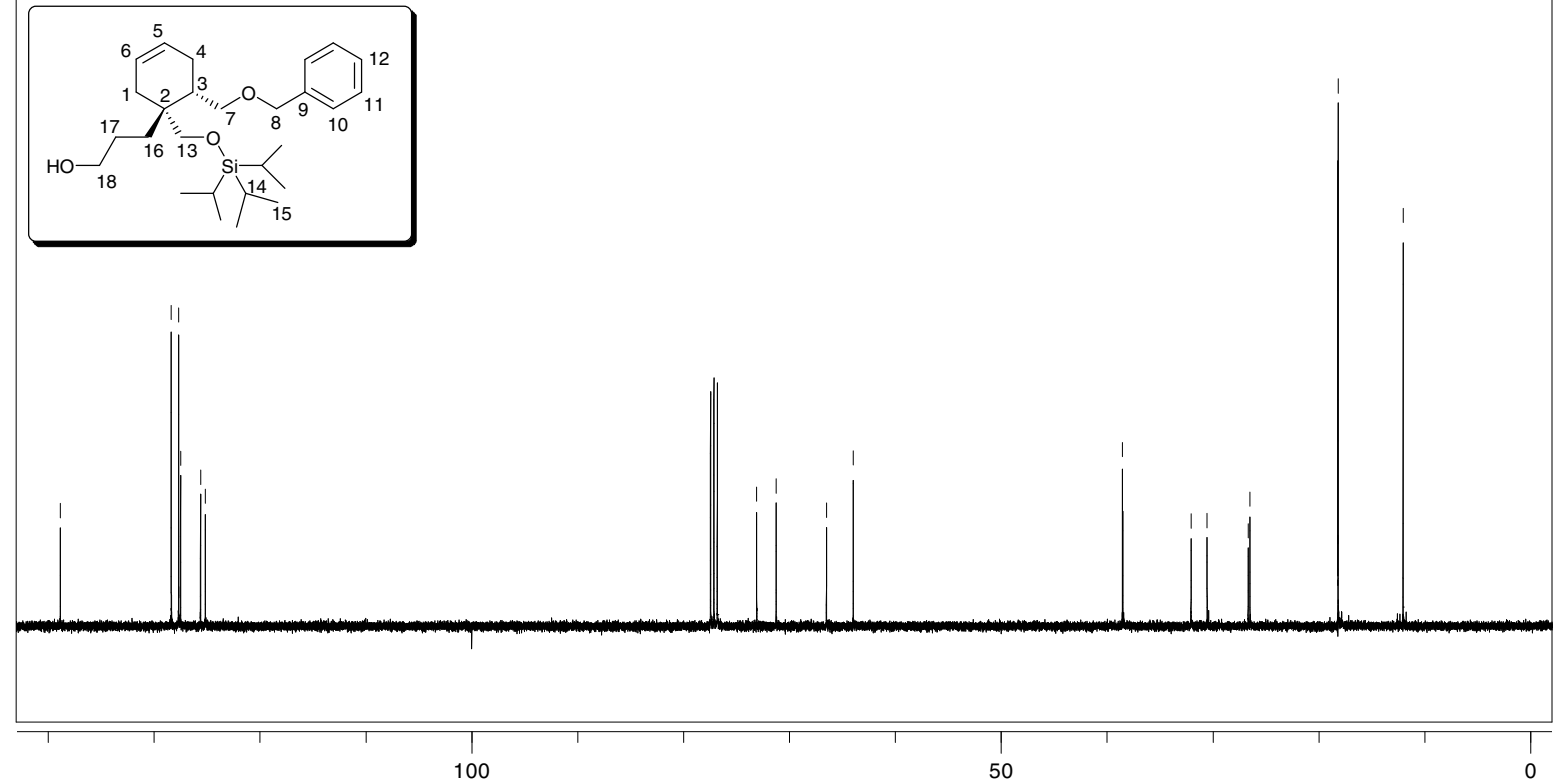

ppm (t1) 


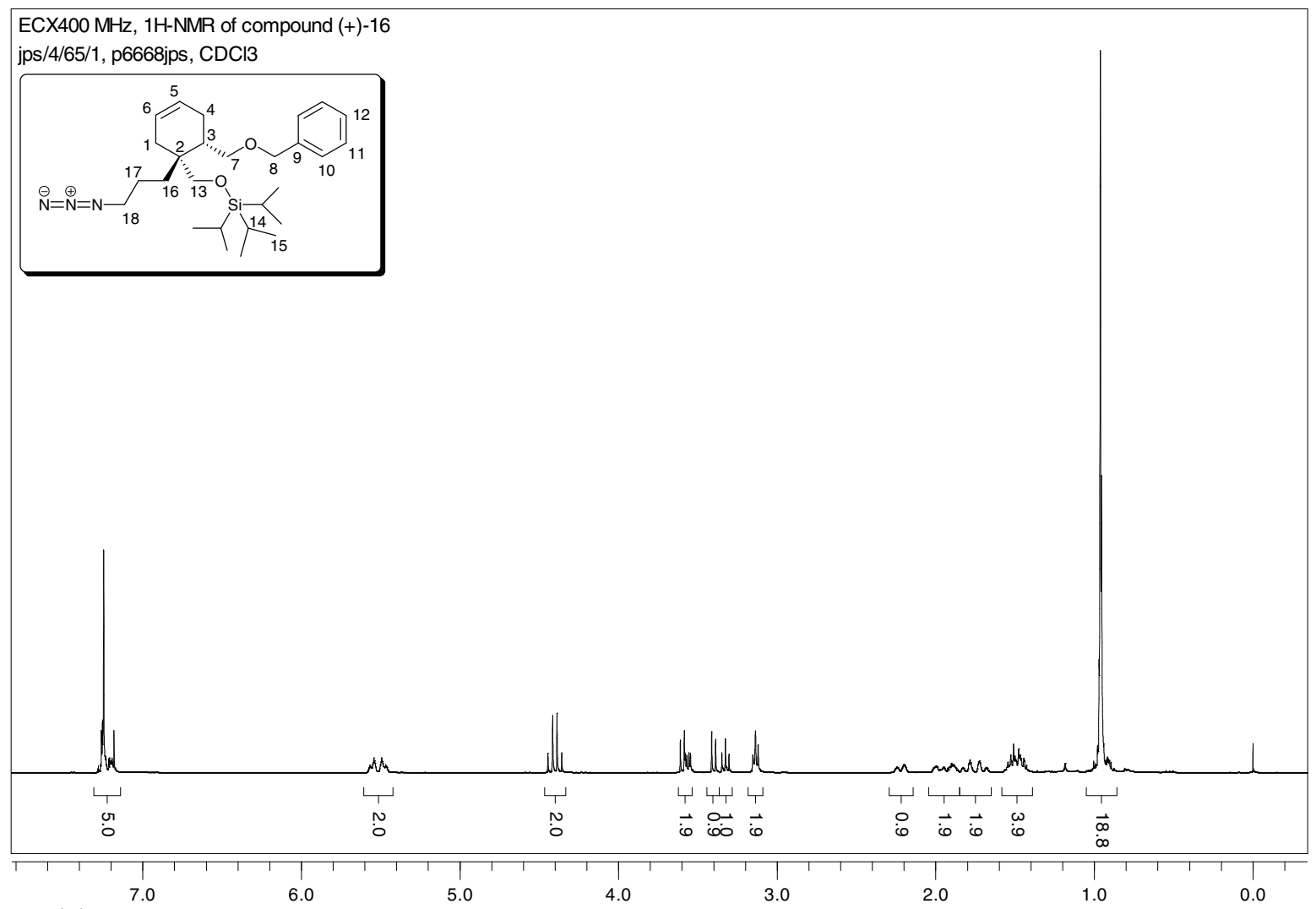

ppm (t1)

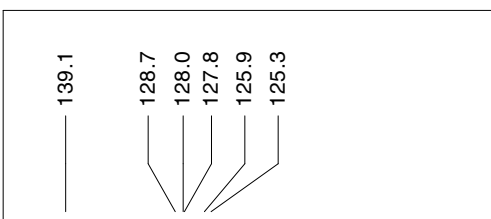

ECX $400 \mathrm{MHz}, 13 \mathrm{C}-\mathrm{NMR}$ of compound (+)-16

jps/4/65/1, p6669jps, CDCl3
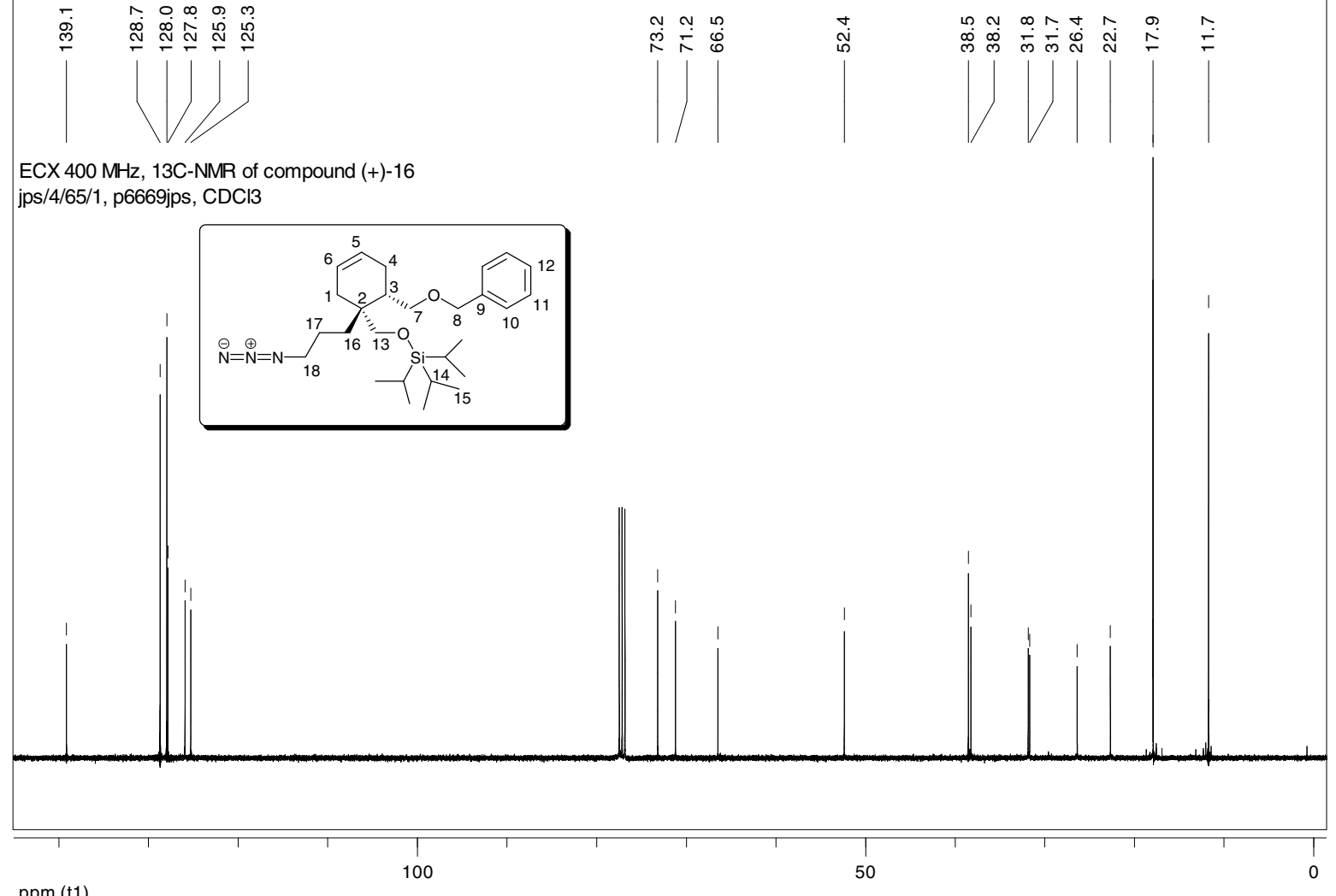

ppm (t1) 


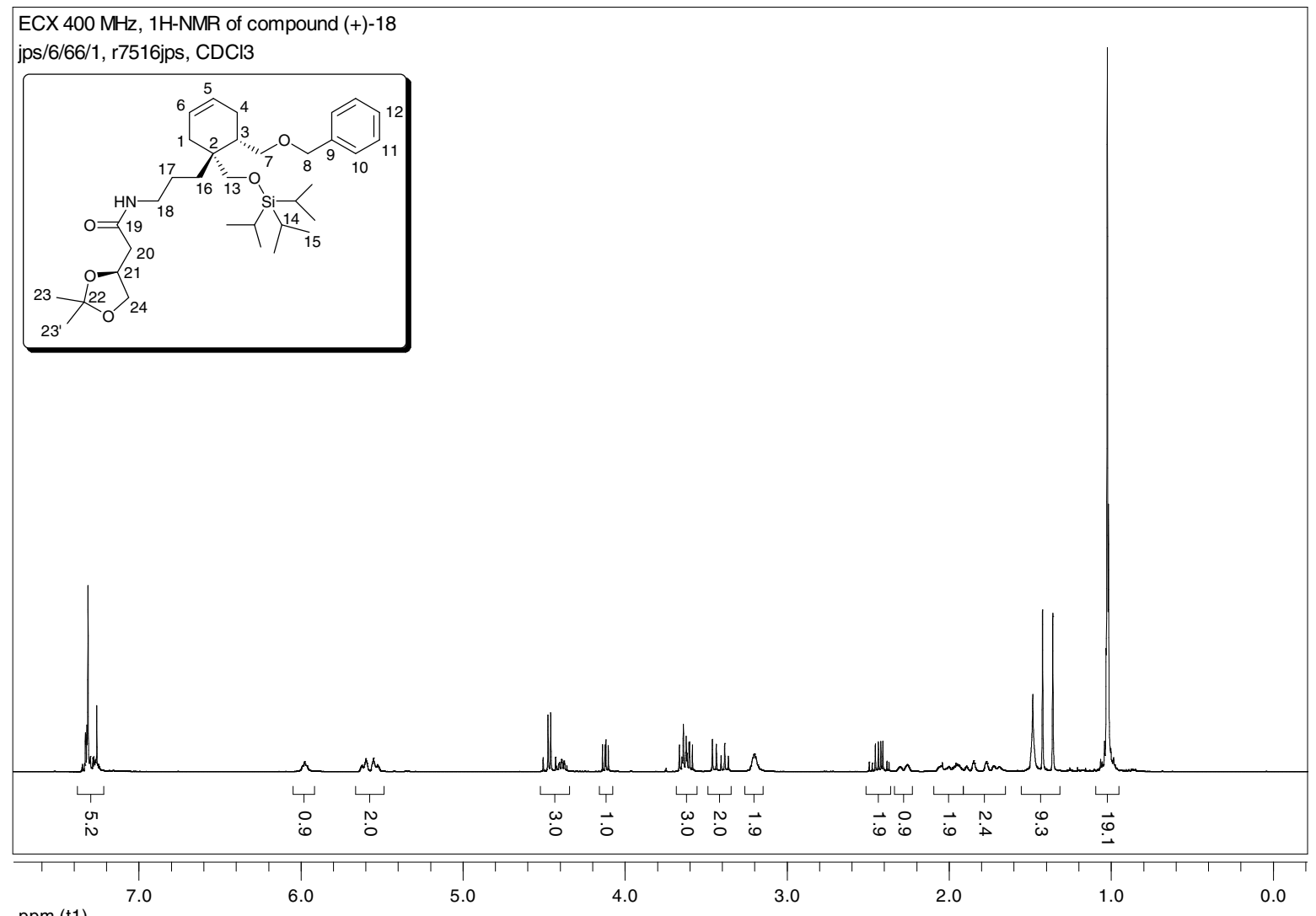

ppm (t1)
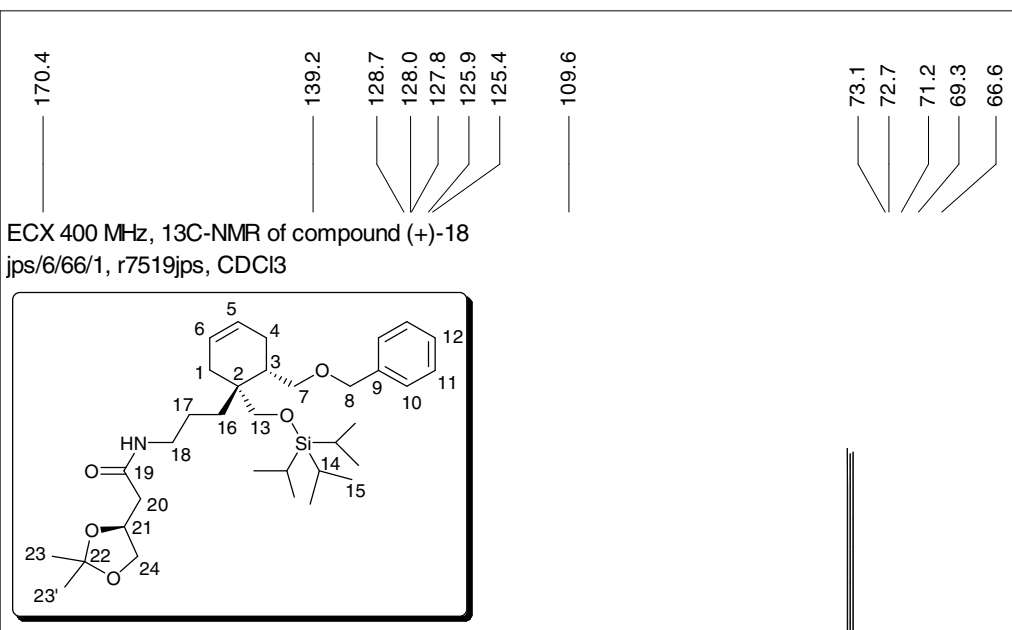

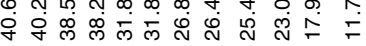

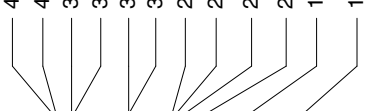

ECX $400 \mathrm{MHz}, 13 \mathrm{C}-\mathrm{NMR}$ of compound (+)-18 jps/6/66/1, r7519jps, CDCl3

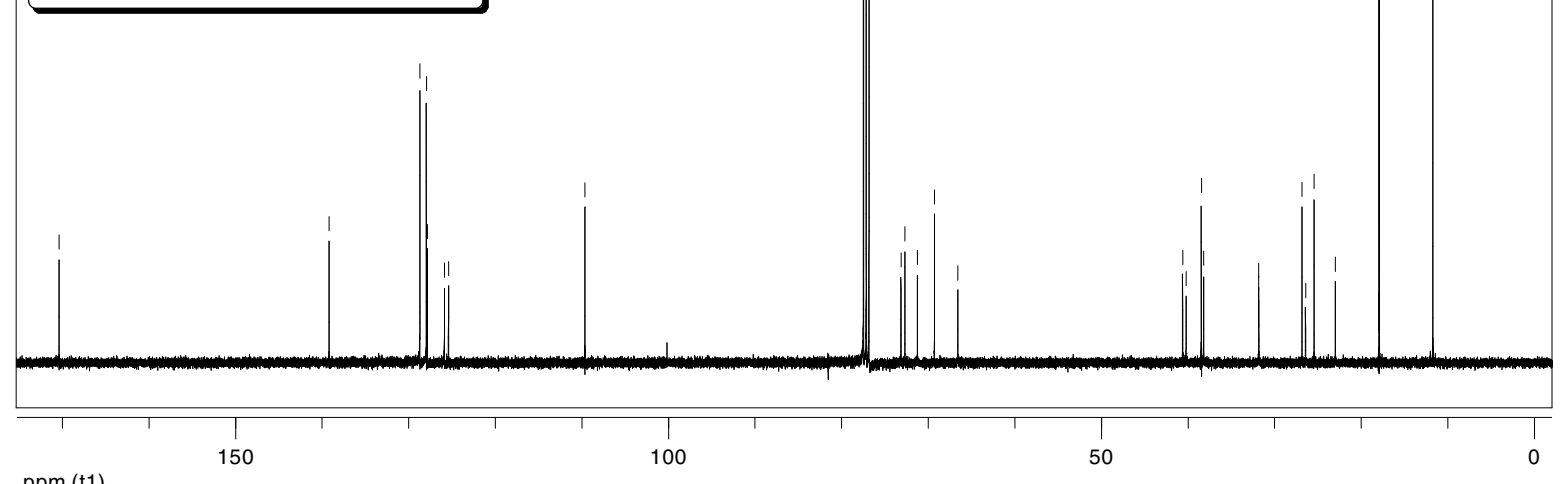



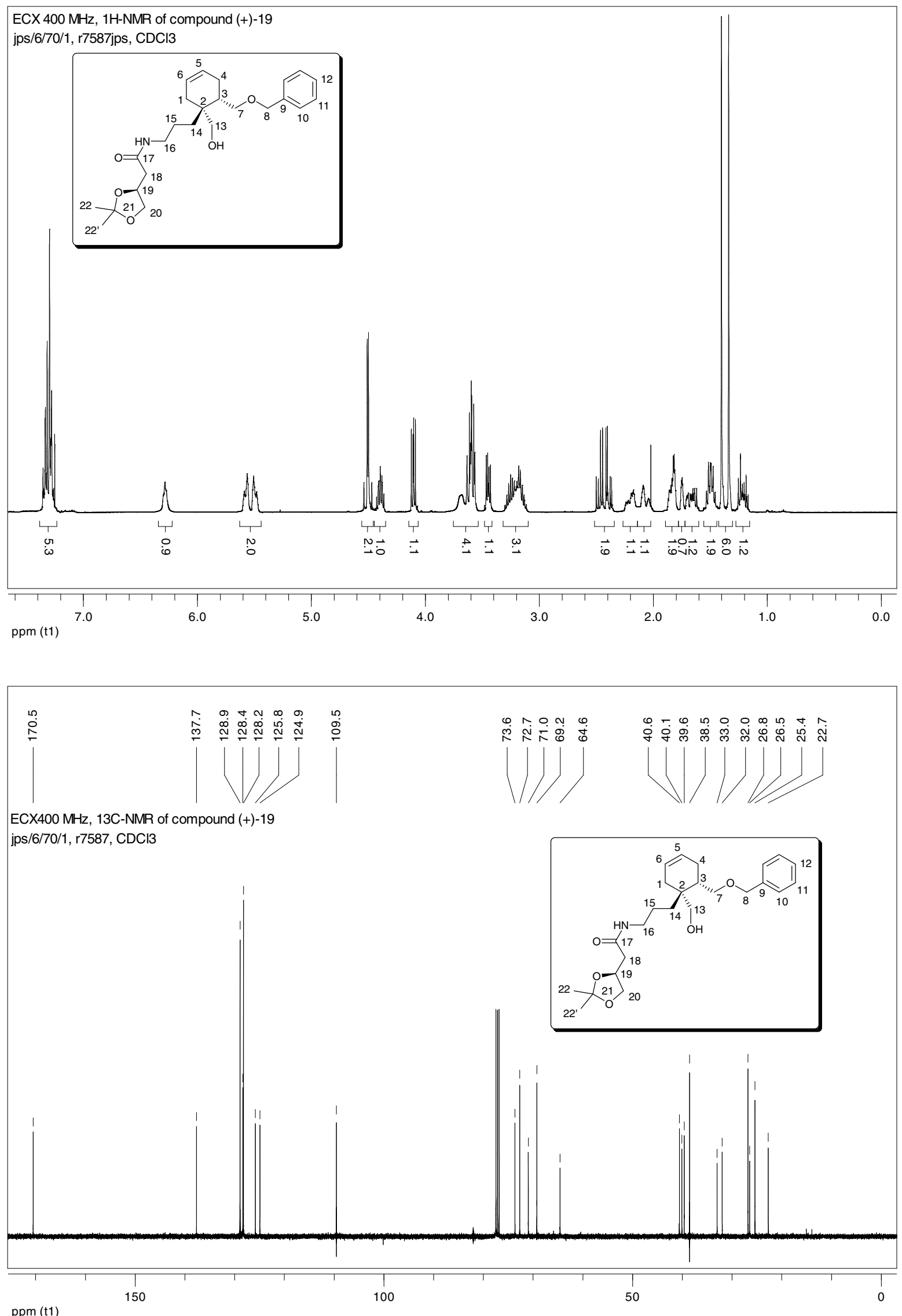


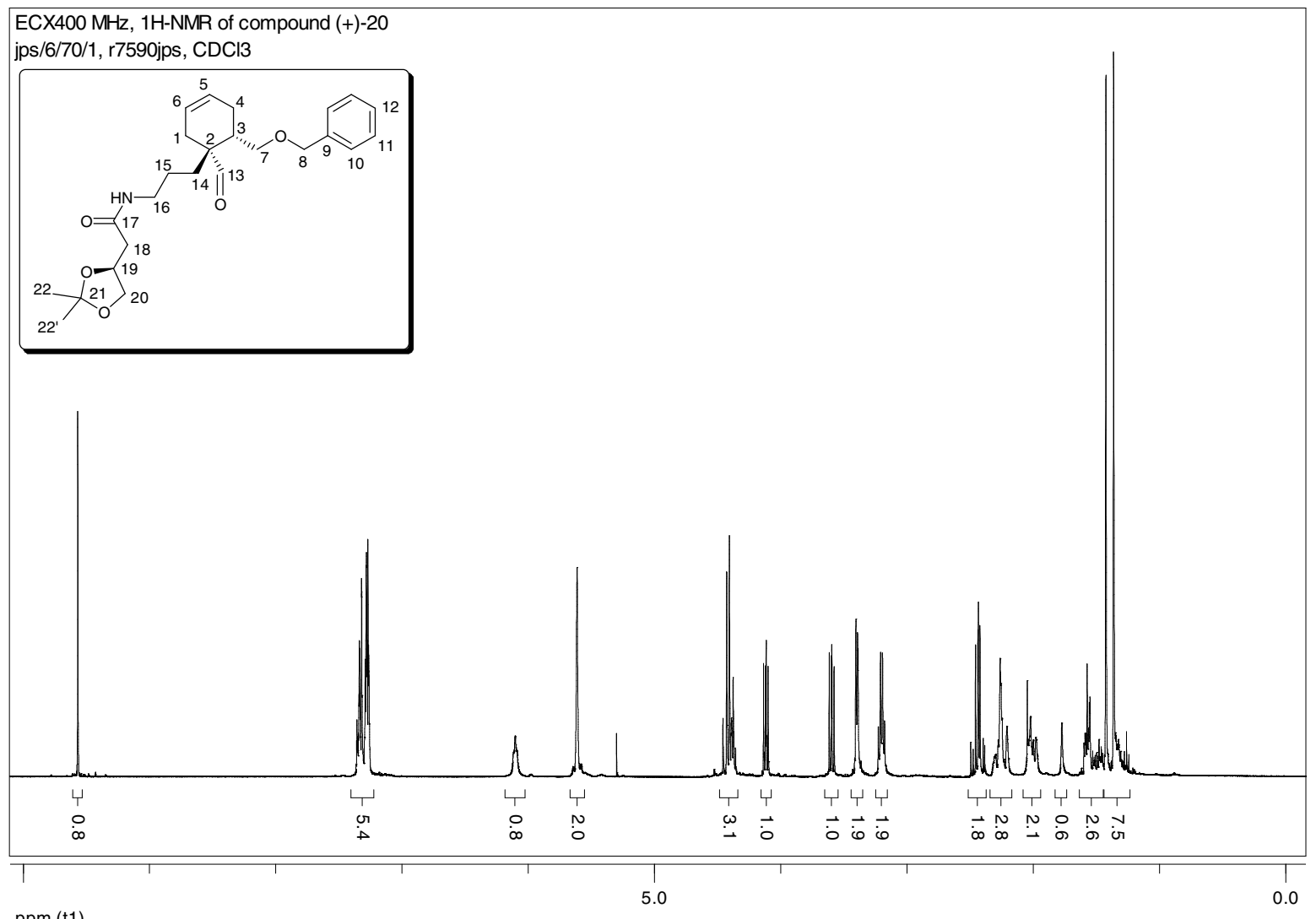

ppm (t1)
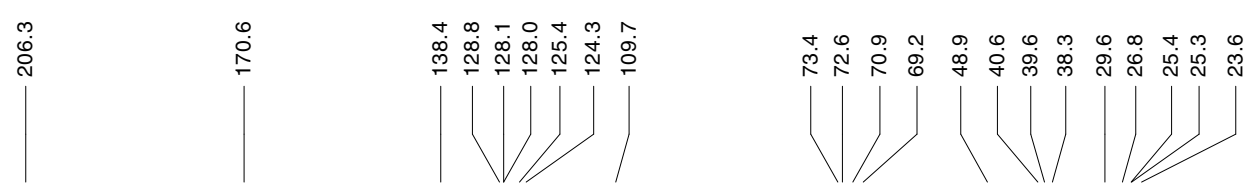

ECX400 MHz, 13C-NMR of compound (+)-20 jps/6/70/1, r7592jps, $\mathrm{CDCl} 3$
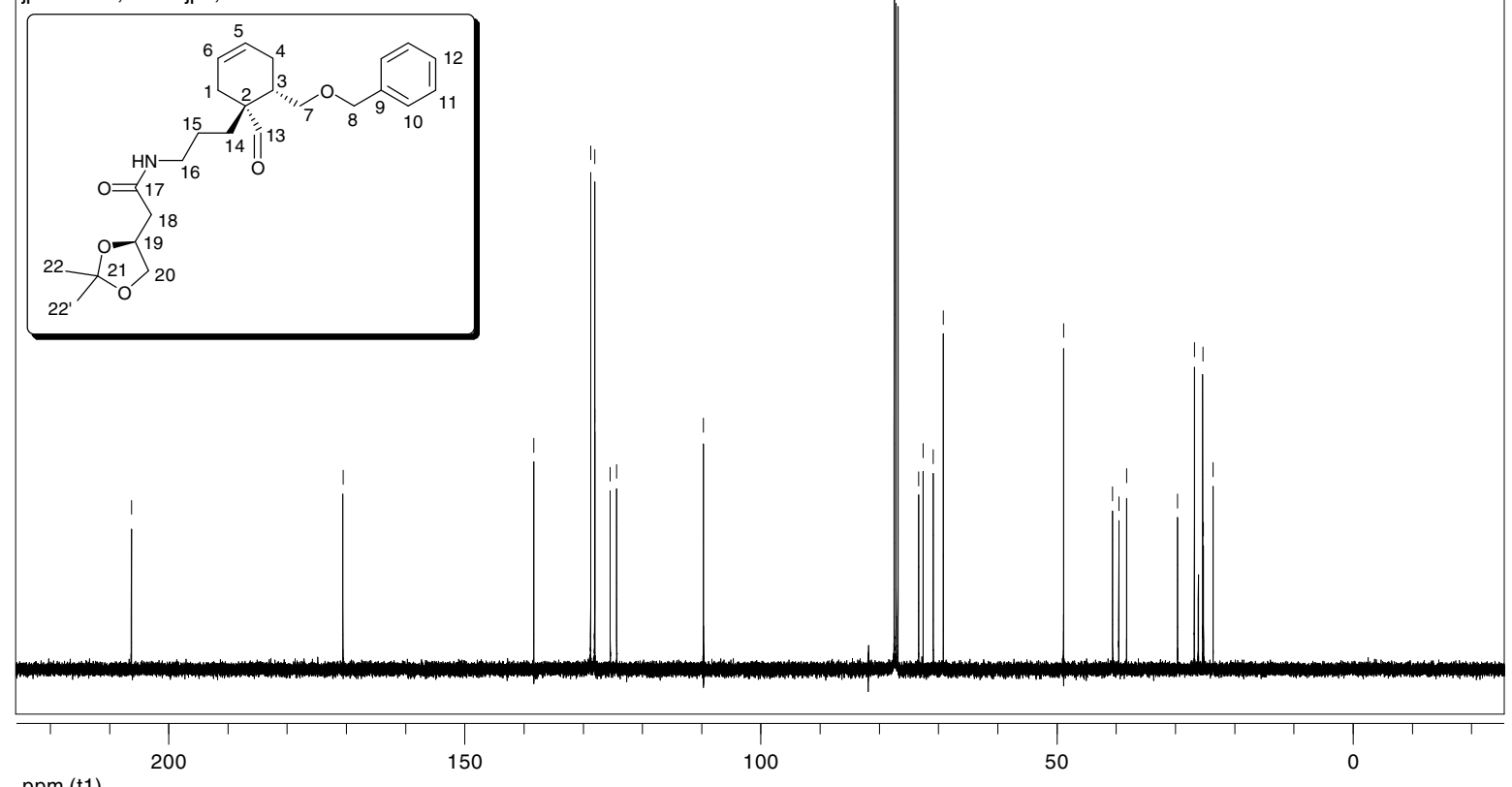


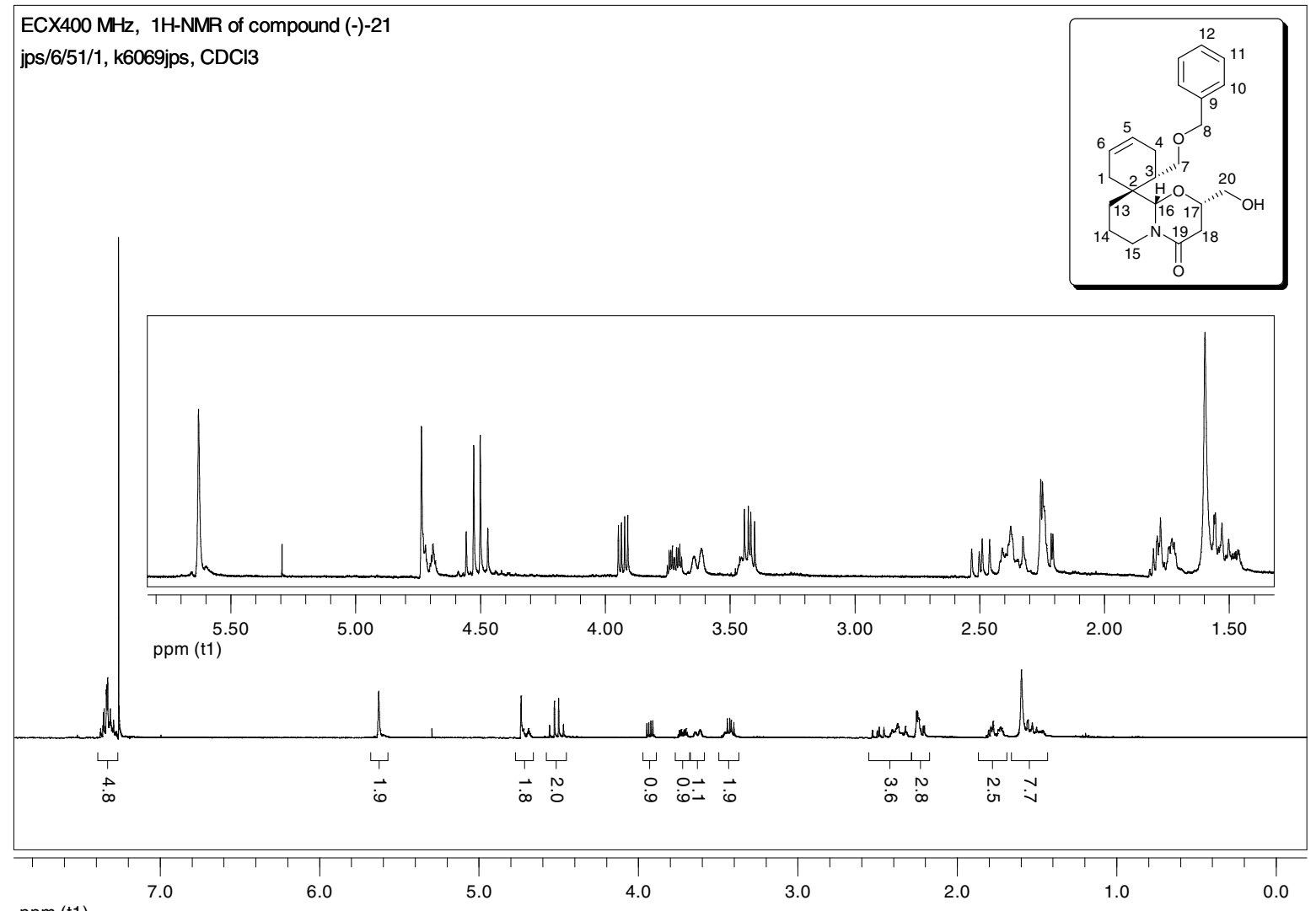

ppm (t1)

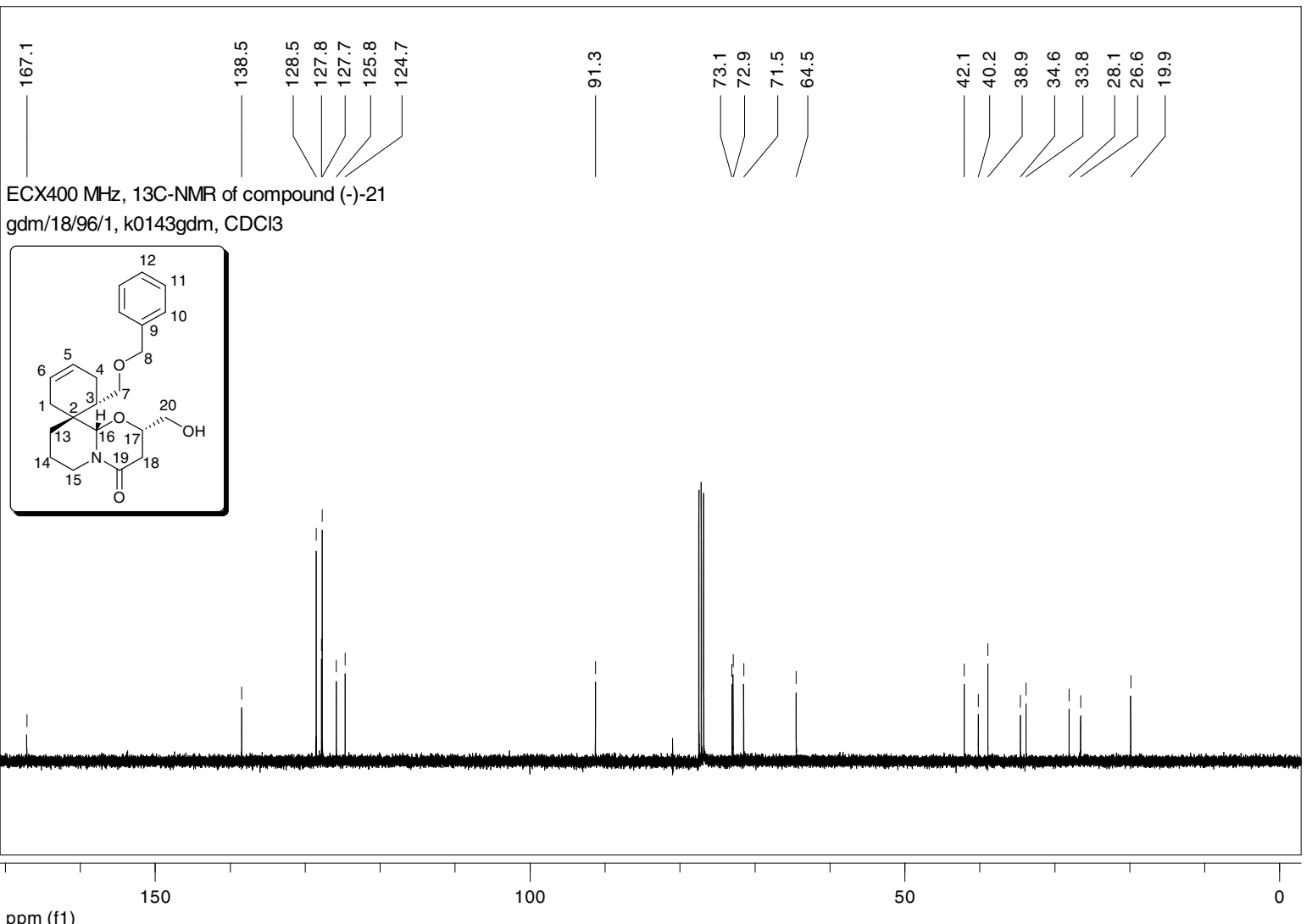



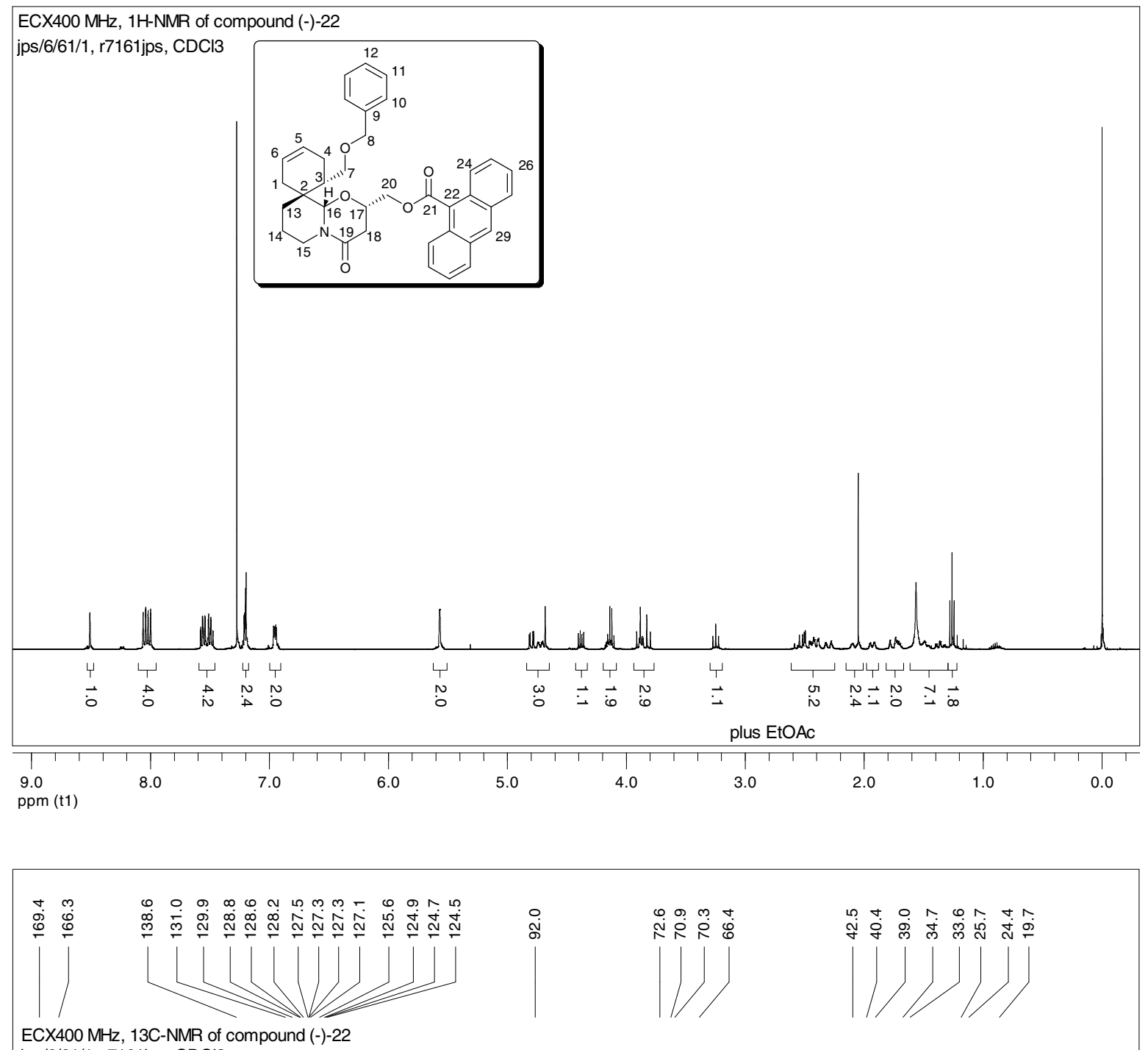

ECX400 MHz, 13C-NMR of compound (-)-22 jps/6/61/1, r7161.jps, $\mathrm{CDCl} 3$

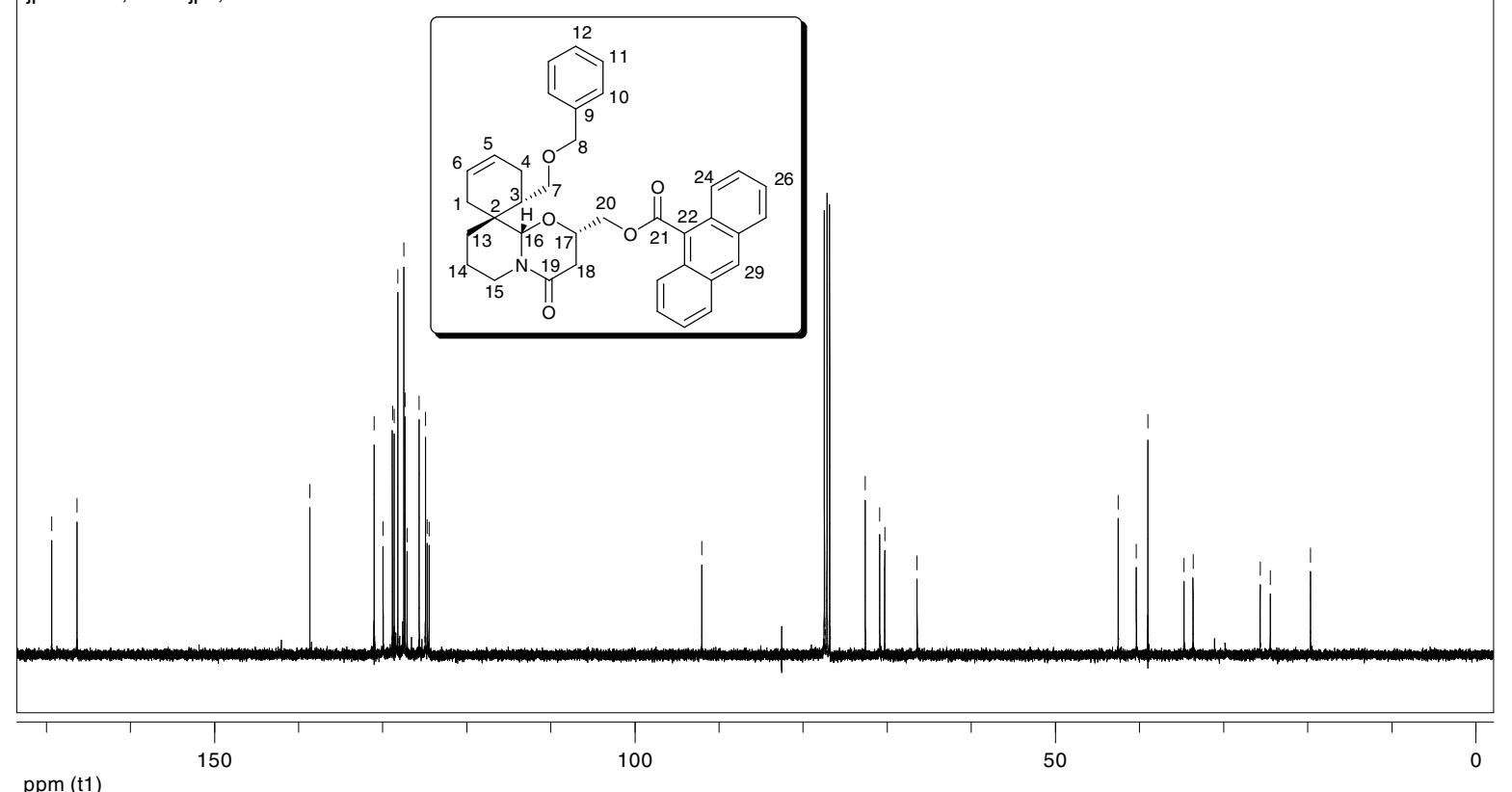




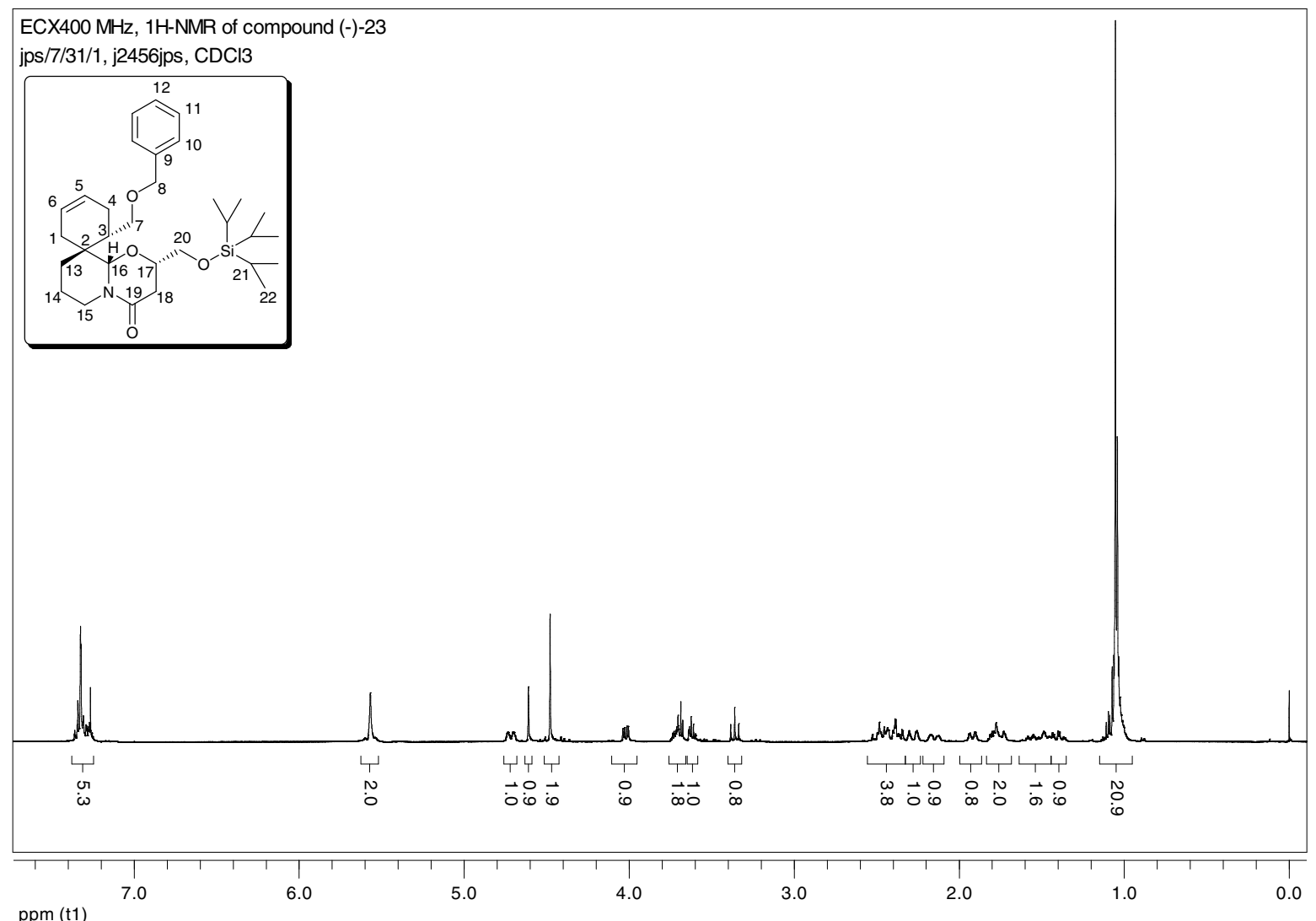

ppm (t1)

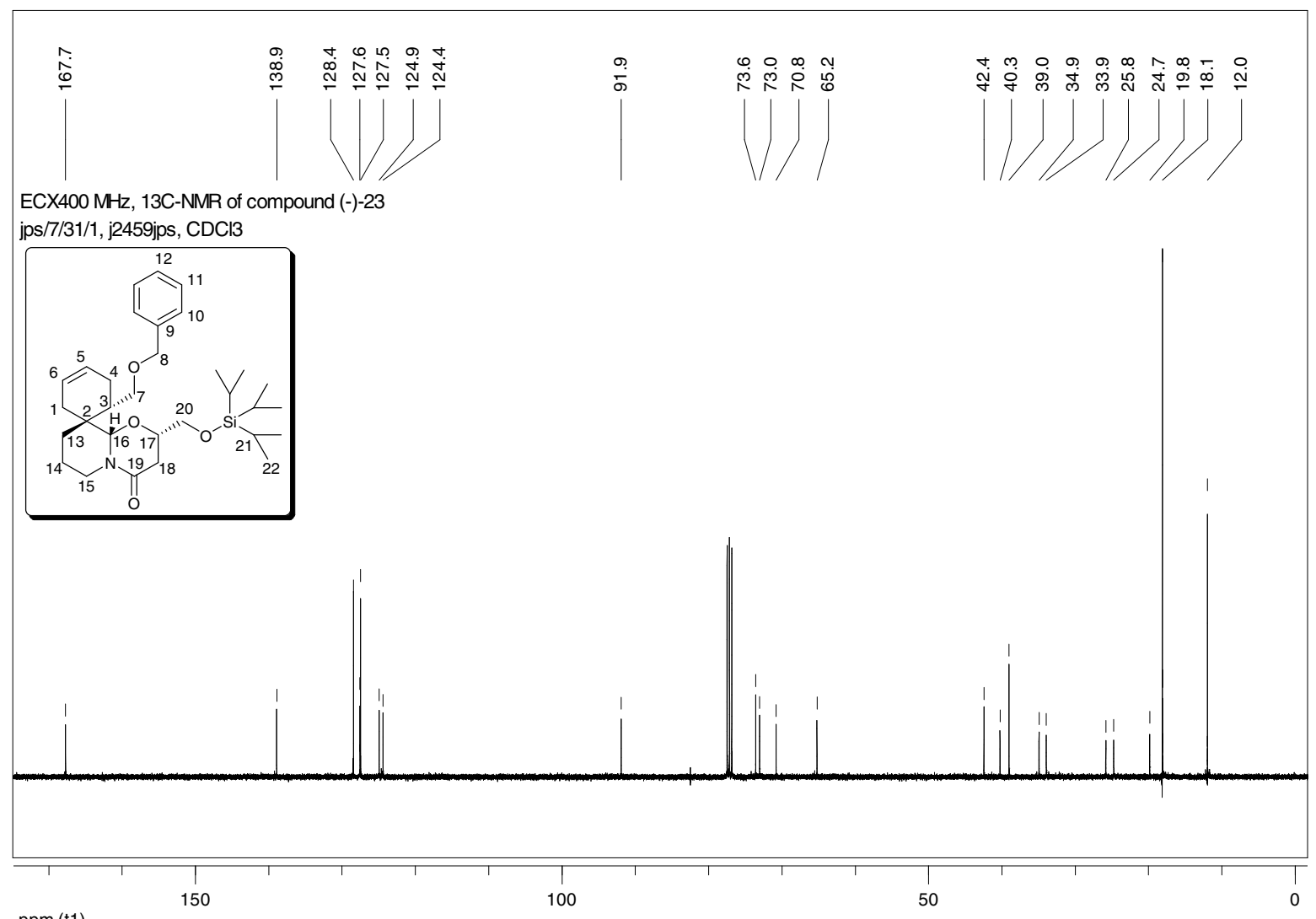

ppm (t1) 


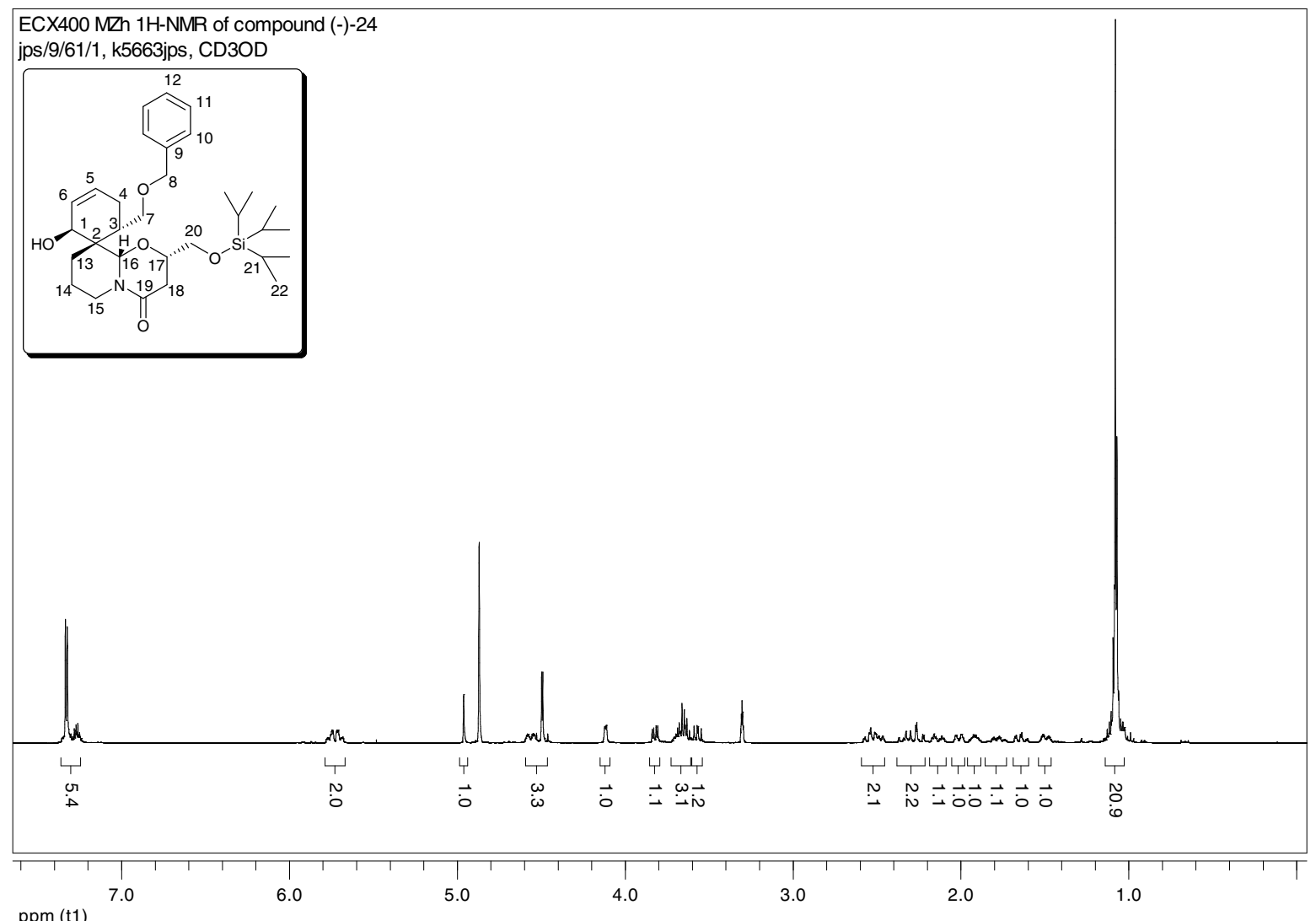

ppm (t1)

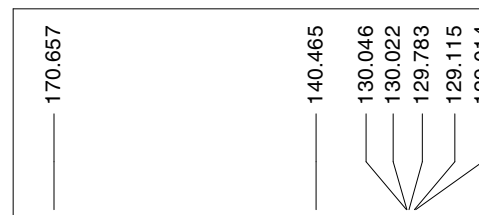

ECX400 MHz 13C-NMR of compound (-)-24

jps/9/61/1, k5662jps, CD3OD
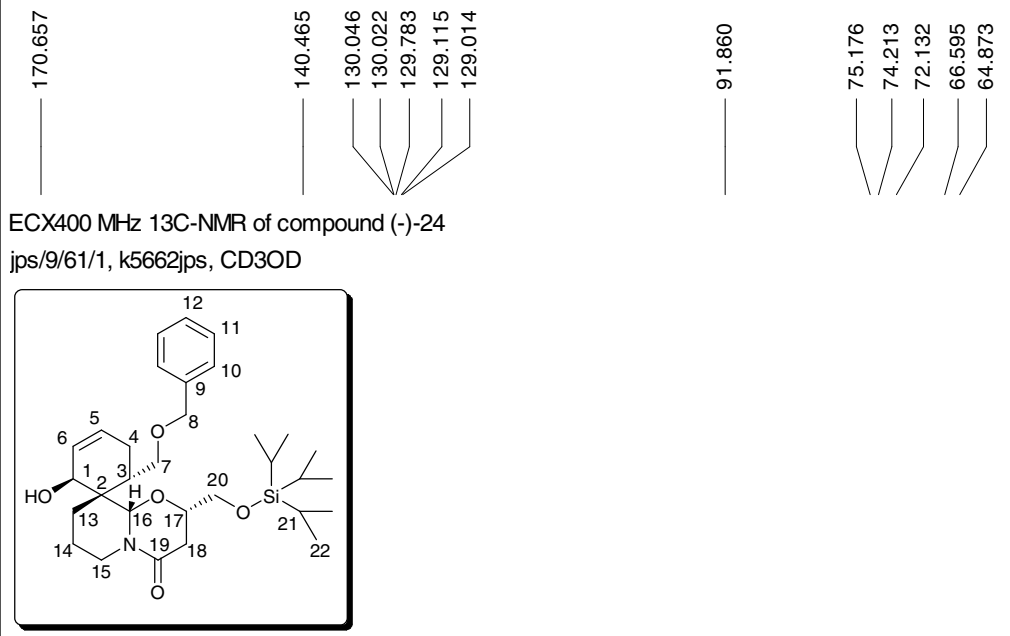

牙

守 守 岗
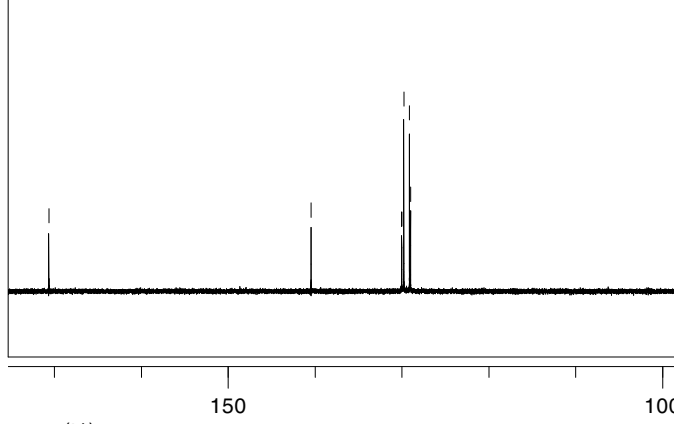

ppm (t1)

100

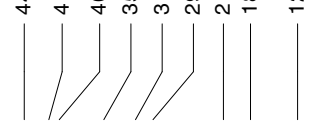




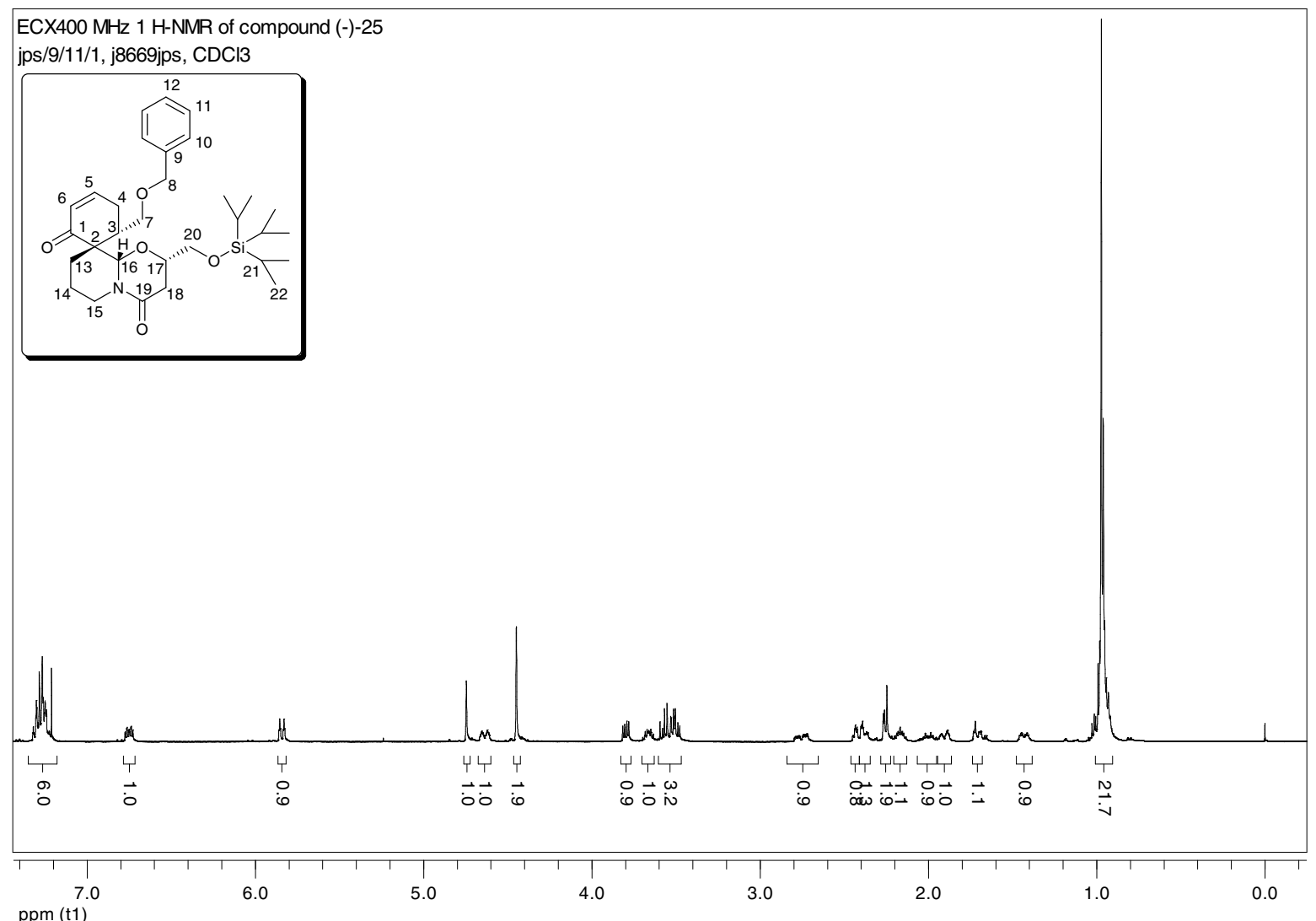

ppm (t1)

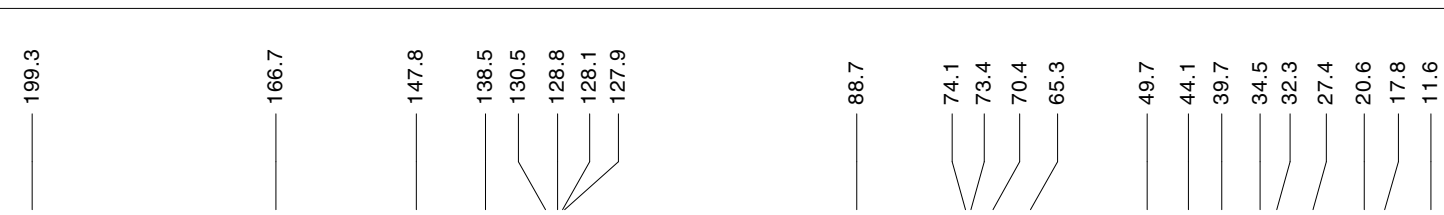

ECX400 MHz, 13C-NMR of compound (-)-25 jps/9/11/1, j8668jps, $\mathrm{CDCl} 3$
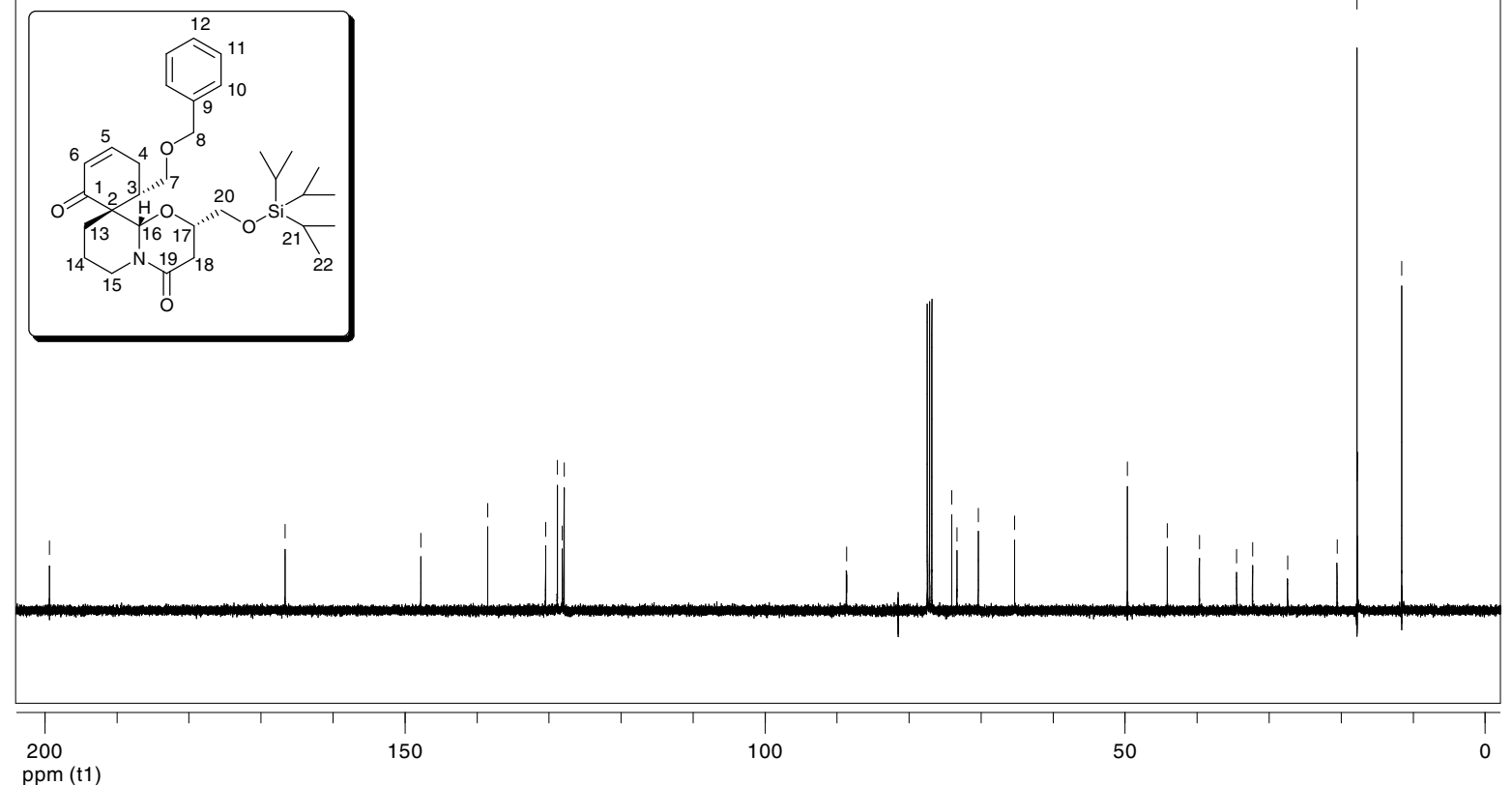

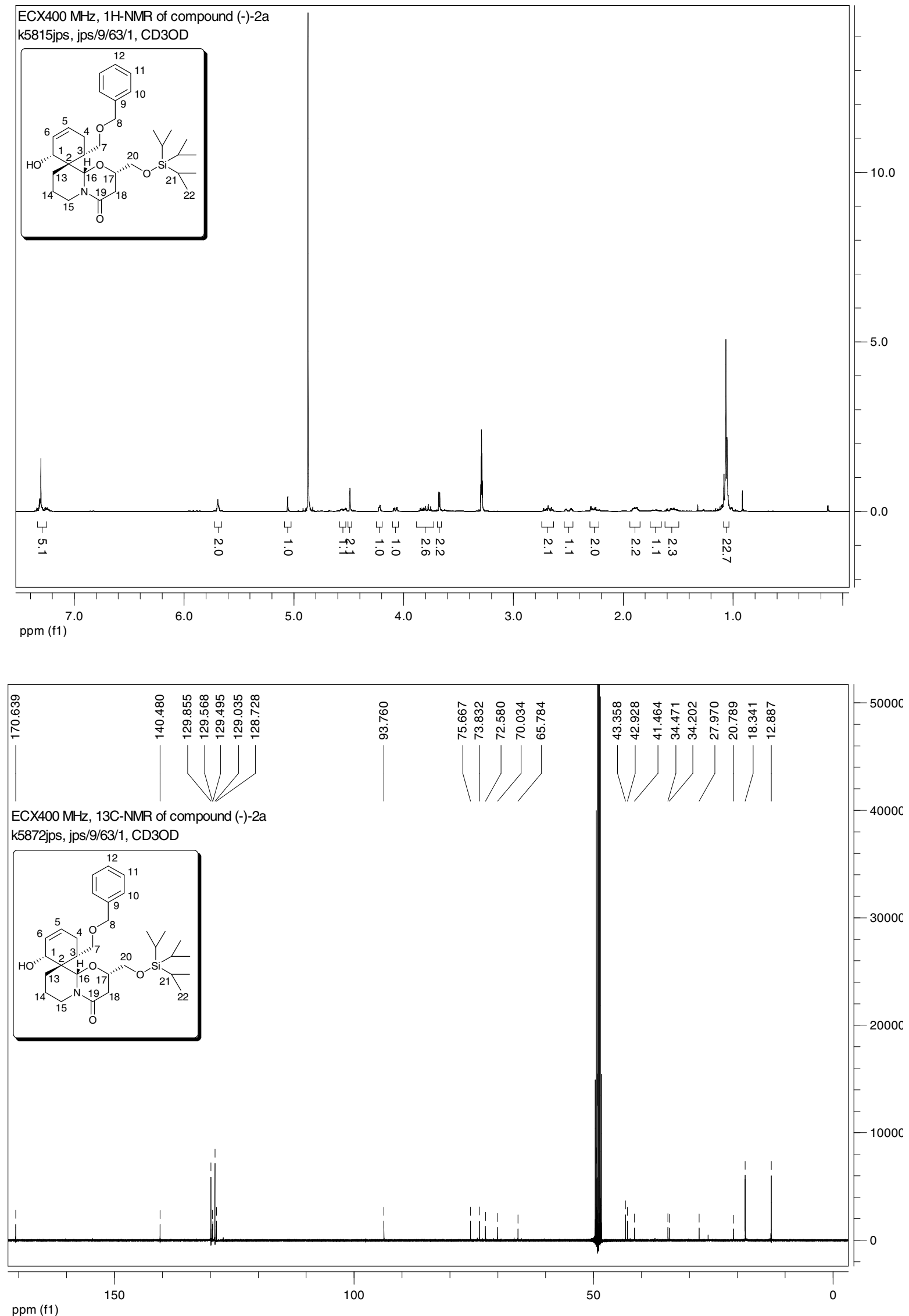